\title{
Generation of a Heteropolycyclic and Sp3-Rich Scaffold for Library Synthesis from a Highly Diastereoselective Petasis/DielsAlder and ROM-RCM Reaction Sequence
}

Flagstad, Thomas; Azevedo, Carlos; Troelsen, Nikolaj Sten; Min, Geanna; Macé, Yohan; Willaume, Anthony; Guilleux, Rachel ; Velay, Mélanie; Bonnet, Karine; Morgentin, Rémy

Total number of authors:

12

Published in:

European Journal of Organic Chemistry

Link to article, DOI:

10.1002/ejoc.201801551

Publication date:

2019

Document Version

Peer reviewed version

Link back to DTU Orbit

Citation (APA):

Flagstad, T., Azevedo, C., Troelsen, N. S., Min, G., Macé, Y., Willaume, A., Guilleux, R., Velay, My., Bonnet, K., Morgentin, R., Nielsen, T. E., \& Clausen, M. H. (2019). Generation of a Heteropolycyclic and Sp -Rich Scaffold for Library Synthesis from a Highly Diastereoselective Petasis/DielsAlder and ROM-RCM Reaction Sequence. European Journal of Organic Chemistry, 2019(5), 1061-1076. https://doi.org/10.1002/ejoc.201801551

\section{General rights}

Copyright and moral rights for the publications made accessible in the public portal are retained by the authors and/or other copyright owners and it is a condition of accessing publications that users recognise and abide by the legal requirements associated with these rights.

- Users may download and print one copy of any publication from the public portal for the purpose of private study or research.

- You may not further distribute the material or use it for any profit-making activity or commercial gain

- You may freely distribute the URL identifying the publication in the public portal 


\title{
Generation of a Heteropolycyclic and $\mathrm{Sp}^{3}$-Rich Scaffold for Library Synthesis from a Highly Diastereoselective Petasis/Diels- Alder and ROM-RCM Reaction Sequence
}

\author{
Thomas Flagstad, ${ }^{[a]}$ Carlos M. G. Azevedo, ${ }^{[a]}$ Nikolaj S. Troelsen, ${ }^{[a]}$ Geanna K. Min,,${ }^{[a]}$ Yohan Macée, ${ }^{[b]}$ \\ Anthony Willaume, ${ }^{[b]}$ Rachel Guilleux, ${ }^{[b]}$ Mélanie Velay, ${ }^{[b]}$ Karine Bonnet, ${ }^{[b]}$ Remy Morgentin, ${ }^{[b]}$ Thomas \\ E. Nielsen, ${ }^{*[c]}$ Mads H. Clausen*[a]
}

Abstract: Efficient access to diverse screening compounds with desirable, lead-like properties can be a bottleneck in early drug discovery and chemical biology. Here we present an efficient, rapid route to three structurally distinct classes of compounds $(A-C)$ from a single precursor, which in turn is available through a one-pot Petasis 3-component-reaction-Diels-Alder cascade reaction. We demonstrate the versatility of the approach through the synthesis of 35 exemplary compounds from the three classes, as well as by the production of 2188 final compounds, which have been included in the Joint European Compound Library of the European Lead Factory.

\section{Introduction}

Accessing new drug candidates through high-throughput screening (HTS) has become an increasingly difficult challenge primarily due to the lack of investigatory efforts into compounds inhabiting a different chemical space. ${ }^{[1]}$ The libraries typically generated for screening often bear similar chemical topology, flat in structure possessing mostly $\mathrm{sp}^{2}$-hybridized carbon atoms making it more challenging to discover new biological activity. ${ }^{[2]}$ Thus, it has become necessary to increase efforts towards screening simple natural product like and $\mathrm{sp}^{3}$-rich cores through diversity-oriented synthesis. ${ }^{[3]}$ However, routine syntheses of such $\mathrm{sp}^{3}$-rich molecules with stereochemical control for use in screening library productions remains a significant challenge..$^{[4]}$ The European Lead Factory initiative addresses this developing issue by targeting natural product-like libraries, ${ }^{[5]}$ while providing production feasible routes from cheap and commercially available building blocks in order to access scaffolds that are $\mathrm{sp}^{3}$-rich with 3-5 functional group handles. ${ }^{[6]}$ These handles can be further functionalized to provide a large library of compounds to be screened for biological activity.

We recently published the synthesis of a $\mathrm{sp}^{3}$-rich library based on a 3-component Petasis/Diels-Alder strategy. ${ }^{[7]}$ This sequence in

[a] Dr. T. Flagstad, Dr. C. M. G. Azevedo, N. S. Troelsen, Dr. G. K. Min, Prof. Dr. M. H. Clausen, Center for Nanomedicine \& Theranostics, Department of Chemistry Technical University of Denmark Kemitorvet 207, DK-2800, Kongens Lyngby, Denmark, mhc@kemi.dtu.dk, www.kemi.dtu.dk/mhc

[b] Dr. Y. Macé, Dr. A. Willaume, Dr. R. Guilleux, Dr. M. Velay, Dr. K. Bonnet, Dr. R. Morgentin, EDELRIS 115 Avenue Lacassagne, F-69003, France

[c] Prof. Dr. T. E. Nielsen, Singapore Centre on Environmental Life Science Engineering Nanyang Technological University Singapore 637551, Singapore and Costerton Biofilm Center, Department of Immunology and Microbiology, University of Copenhagen Copenhagen DK-2200, Denmark ten@sund.ku.dk

combination with two ring opening/ring closing metathesis (ROM/RCM) cascade reactions enabled us to access different, diverse and $\mathrm{sp}^{3}$-rich scaffolds. Herein we present the synthesis of a new library that has led to the production of 2188 HTS compounds.

\section{Results and Discussion}

In order to rapidly build a compact functionalized core system, we focused on a synthetic route which includes a highly diastereoselective 3-component Petasis/Diels-Alder tandem reaction from allylic amines (1a), furylboronic acids (1b), and $\alpha$ hydroxylated aldehydes (1c) (Scheme 1). This reaction manifold was first reported by Norsikian, Beau, and co-workers and later further explored by us. ${ }^{[8]}$ We envisioned that this strategy would allow us to access different libraries depending on the $\mathrm{N}$ substituent on the boronic acid building block $\mathbf{1 b}$ and the $\mathrm{R}^{3}$ substituent on the aldehyde 1c as a single diastereomer. Based on this scaffold 1 , we wish to report the generation of three different sub-libraries (library A-C) using robust and scalable chemistry.

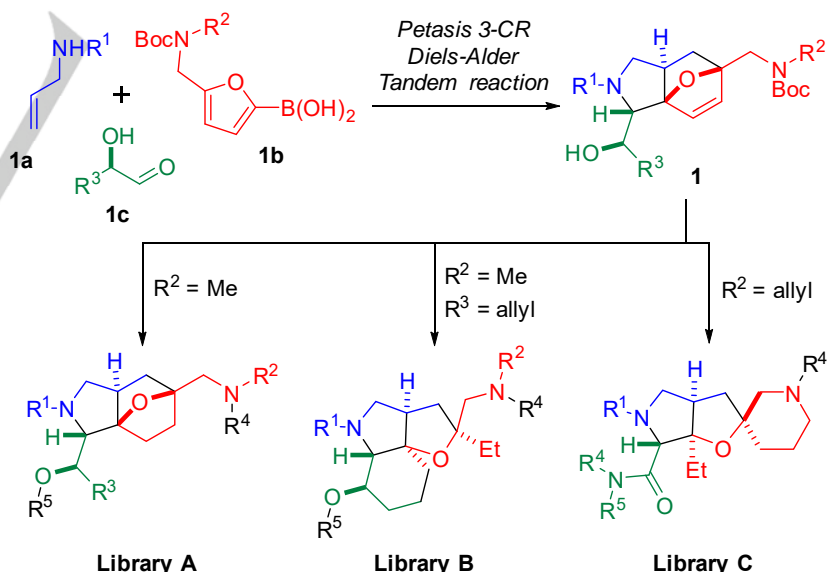

Scheme 1: Synthetic strategy for the generation of libraries $A-C$ from tandem Petasis 3-CR/Diels-Alder product 1.

The Petasis 3-component reaction ${ }^{[9]}$ followed by an intramolecular Diels-Alder reaction ${ }^{[10]}$ was introduced early in the scaffold synthesis to provide the core skeleton with the stereocenters pre-set for the library generation. The benzyl group, used in lieu of $\mathrm{R}^{1}$ during scaffold construction, was strategically employed to serve as an orthogonal protecting group to the Bocprotected amine tethered to the furylboronic acid $\mathbf{1 b}$. This would allow for selective late stage deprotection and functionalization. Boronic acids $\mathbf{3}$ and $\mathbf{4}$ were obtained through a reductive 
amination of commercially available 5-formyl-2-furanylboronic acid (2) and a primary amine followed by a subsequent Boc protection (Scheme 2 ). The boronic acid obtained could not easily be purified using conventional methods, so it was directly employed in the Petasis/Diels- Alder reaction after extraction to afford key intermediates 5 (library A), 6 (library B), and 7 (library C) in $75 \%, 31 \%$, and $44 \%$ isolated yields, respectively - each as a racemic mixture of a single diastereomer.

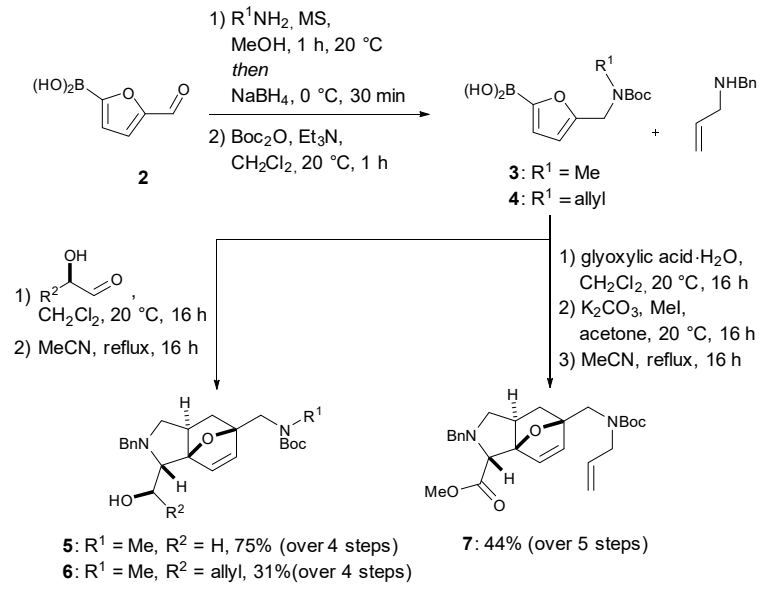

Scheme 2: Petasis 3-component reaction accessing key intermediates 5-
Library A was constructed from scaffold $\mathbf{5}$ through a series of deprotection and derivatisation steps of the three available handles as depicted in Scheme 3. The primary alcohol of $\mathbf{5}$ could be functionalized using Mitsunobu conditions ${ }^{[11]}$ to provide aryl ether 8. $N$-Functionalization of the two amines was then performed, first by Boc-deprotection of the exocyclic amine followed by either acylation or sulfonylation, and then benzyl deprotection of the pyrrolidine amine followed by reductive alkylation, sulfonylation, or acylation to provide 9-14 in high yields over four steps. Alternatively, subjecting 5 to reductive conditions followed by reductive alkylation of the resulting amine provided 2fluorobenzyl amine $\mathbf{1 5}$ in $84 \%$ isolated yield over two steps. Subsequently, arylation of the primary alcohol, like described above, followed by Boc-deprotection of the exocyclic amine and either alkylation or acylation gave $\mathbf{1 6}$ and $\mathbf{1 7}$ in excellent yields over three steps.

The synthesis of library B core scaffold 18 relied on a metathesis cascade reaction ${ }^{[12]}$ from 6 (Scheme 4). Upon subjection to Grubbs' $2^{\text {nd }}$ generation catalyst (Grubbs II) under acidic conditions, the 7-oxabicyclo[2.2.1] hept-2-ene underwent a $\mathrm{ROM} / \mathrm{RCM}$ cascade reaction with the distal monosubstituted alkene to provide core 18 in $86 \%$ yield. Olefin reduction and pyrrolidine amine benzyl deprotection was performed using palladium on carbon and ammonium formate. The pyrrolidine amine could then be selectively alkylated over the exocyclic secondary amine using reductive alkylation conditions. Finally, the exocyclic secondary amine was derivatised by reaction with an acid chloride or an isocyanate to provide 19 and 20 , respectively, in high yields over three steps.

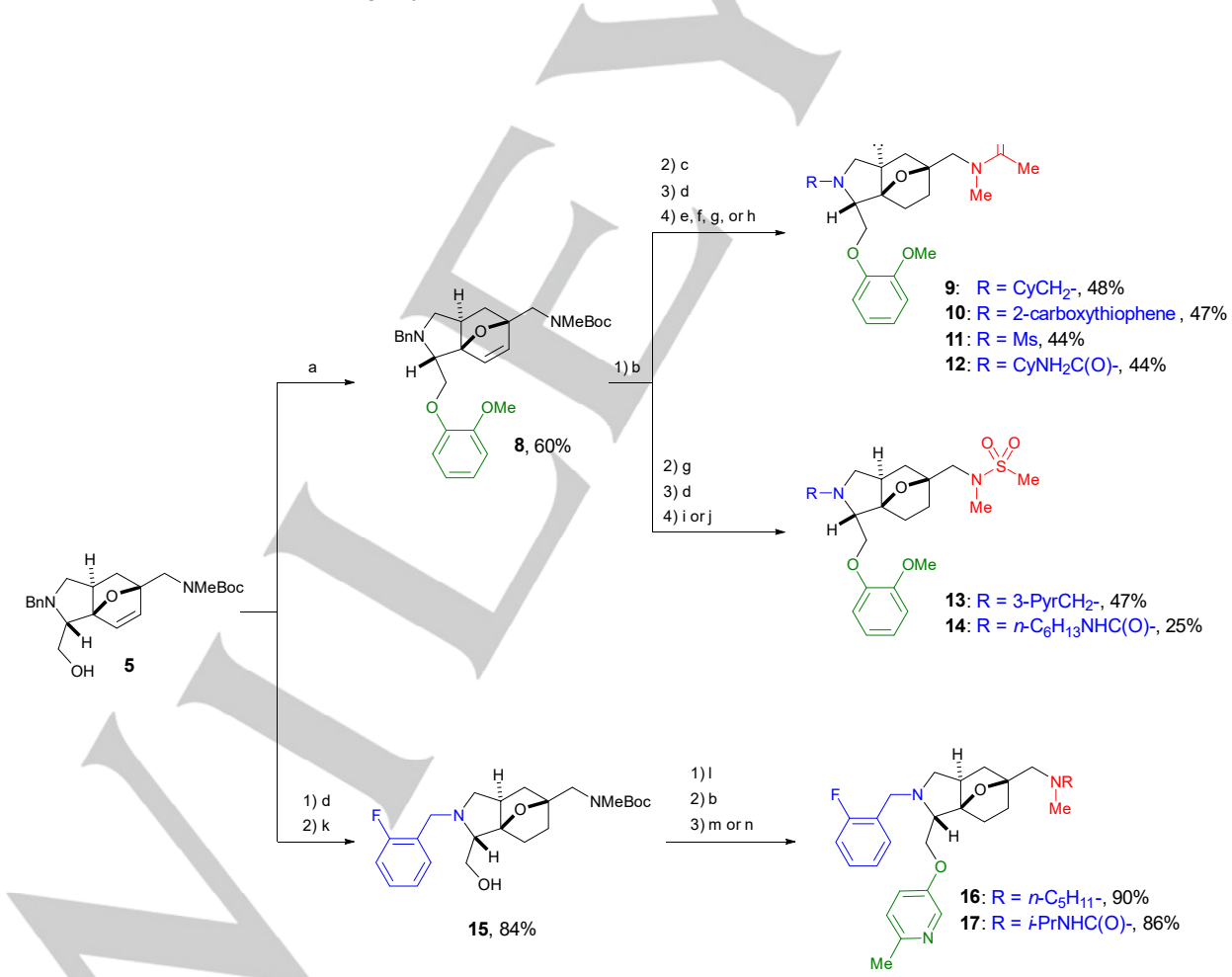

Scheme 3: Synthesis of library A through $\mathrm{N}$-functionalization of the pyrrolidine amine, arylation of the primary alcohol using Mitsunobu conditions, and exocyclic amine functionalisation. Reagents and conditions: (a) 2-methoxyphenol, $\mathrm{PPh}, \mathrm{DEAD}, \mathrm{THF}, 0 \rightarrow 20^{\circ} \mathrm{C}, 18 \mathrm{~h}$. (b) TFA, $\mathrm{CH}_{2} \mathrm{Cl}_{2}, 20^{\circ} \mathrm{C}, 1 \mathrm{~h}$. (c) $\mathrm{AcCl}$, DIPEA, DMF, $20^{\circ} \mathrm{C}, 18 \mathrm{~h}$. (d) $5 \% \mathrm{Pd} / \mathrm{C}, \mathrm{HCOONH}_{4}, \mathrm{MeOH}$, reflux, $1.5 \mathrm{~h}$. (e) $\mathrm{CyCHO}, \mathrm{NaBH}_{3} \mathrm{CN}, \mathrm{HOAc}, \mathrm{MeOH}, 20^{\circ} \mathrm{C}$, $18 \mathrm{~h}$. (f) thiophene-2carbonyl chloride, DIPEA, DMF, $20^{\circ} \mathrm{C}, 18 \mathrm{~h}$. (g) MsCl, DIPEA, DMF, $0{ }^{\circ} \mathrm{C}, 1 \mathrm{~h}$. (h) cyclopentyl isocyanate, DMF, $20^{\circ} \mathrm{C}, 18 \mathrm{~h}$. (i) nicotinaldehyde, $\mathrm{NaBH}_{3} \mathrm{CN}, \mathrm{HOAc}, \mathrm{MeOH}, 20^{\circ} \mathrm{C}, 18$ h. (j) $n$-HexNCO, DMF, $20^{\circ} \mathrm{C}, 18 \mathrm{~h}$. (k) 2-fluorobenzaldehyde, $\mathrm{NaBH}_{3} \mathrm{CN}, \mathrm{HOAc}, \mathrm{MeOH}, 20^{\circ} \mathrm{C}, 18 \mathrm{~h}$. (I) $5-$ hydroxy-2-methylpyridine, $\mathrm{PPh}_{3}$, DEAD, THF, $0 \rightarrow 20^{\circ} \mathrm{C}, 18 \mathrm{~h}$. $(\mathrm{m})$ valeraldehyde, $\mathrm{NaBH}_{3} \mathrm{CN}, \mathrm{HOAc}, \mathrm{MeOH}, 20{ }^{\circ} \mathrm{C}, 18 \mathrm{~h}$. (n) $i-\mathrm{PrNCO}, \mathrm{DMF}, 20^{\circ} \mathrm{C}, 18$ h. 


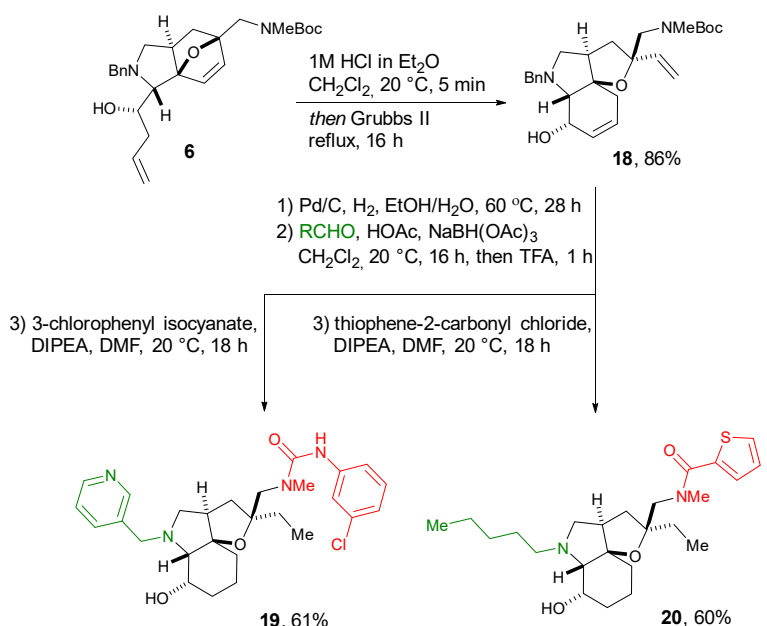

Scheme 4: Library B scaffold synthesis and diversification.

By switching the methyl substituent to an allyl group on the exocyclic amine, library C core scaffold 21 was accessed after treatment of the $\mathrm{HCl}$ salt of 7 with $2 \mathrm{~mol} \%$ of Grubbs II (Scheme $5)$. Ring opening of the internal olefin followed by ring closing metathesis with the tethered allyl amine provided spirocycle $\mathbf{2 1}$ in $83 \%$ isolated yield as a single diastereomer, the stereochemistry being determined in the preceding tandem Petasis/Diels-Alder reaction. Based on 21, library $C$ scaffold diversification was achieved through $\mathrm{N}$-functionalization of both the pyrrolidine and piperidine rings and amidations of the ester. The orthogonal protecting groups allowed for the selective deprotection of either the methyl ester, pyrrolidine amine, or piperidine amine and their subsequent functionalisations. global reduction using hydrogenation and subsequent acylation of the pyrrolidine afforded $\mathbf{2 4}$ in $\mathbf{7 7 \%}$ yield over four steps. Ester hydrolysis and amidation provided morpholine amide $\mathbf{2 5}$ in $60 \%$ yield over two steps. Using this procedure, an additional three compounds, 30, 31, and 32, were likewise synthesised from 24 to give examples of both primary, secondary, and tertiary amides in the $\mathrm{R}^{1}$ handle (Scheme 7 ).

Starting from 22, deprotection of the pyrrolidine ring and global reduction was performed first followed by reductive alkylation to provide 26 in quantitative yield. Subsequent Boc deprotection of the piperidine with TFA followed by sulfonylation afforded $\mathbf{2 7}$ in $93 \%$ over two steps (Scheme 6). Using this deprotection and derivatisation sequence, compounds 33-38 were synthesised to provide examples of acylation and reductive alkylation on both heterocyclic amines (Scheme 7).

The order of functionalization of the two saturated heterocycles can also be reversed. Boc deprotection of the piperidine in $\mathbf{2 3}$ followed by acylation provided $\mathbf{2 8}$ in $\mathbf{8 5 \%}$ yield over two steps. Subsequent hydrogenation deprotected the pyrrolidine amine and globally reduced the olefins. The pyrrolidine amine was then functionalized by sulfonylation to afford 29 in $75 \%$ yield over two steps (Scheme 6). Five additional compounds, 39-44, were synthesised in this fashion using acylation or reductive alkylation (Scheme 7).

The developed strategy was subsequently used to produce 2,188 high-throughput screening compounds for the European Lead Factory. The library showed a high degree of three-dimensionality with an average $\mathrm{Fsp}^{3}$ of 0.72 and good distributions of CLogP, molecular weight, and polar surface area (PSA) (Figure 1). Figure 2 shows a comparison of CLogP vs. molecular weight.
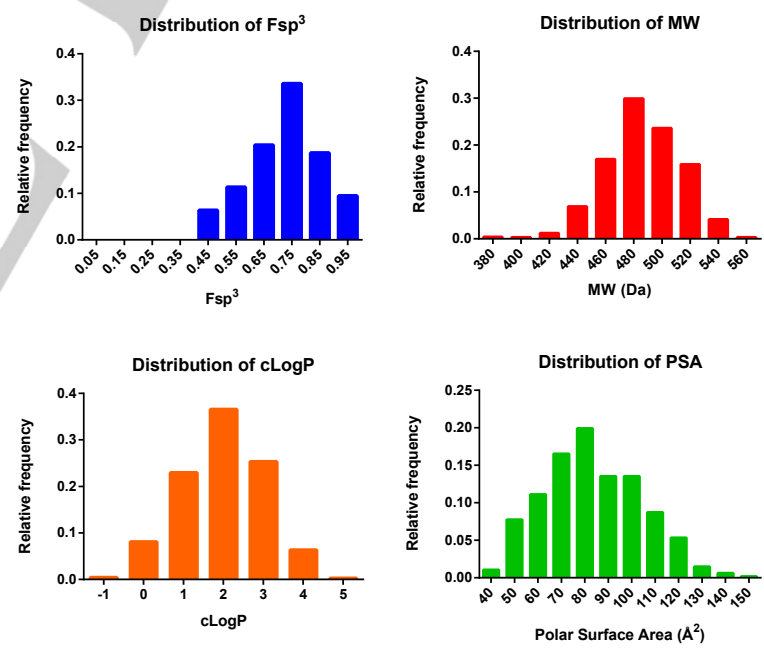

Figure 1: Analysis of the 2,188 synthesised high-throughput screening compounds for the European Lead Factory. Average $\mathrm{Fsp}^{3}=0.72$, average $\mathrm{MW}=486 \mathrm{Da}$, average $\mathrm{cLog} \mathrm{P}=1.98$, and average $\mathrm{PSA}=83.5 \AA^{2}$.
Hydrolysis of the methyl ester using $\mathrm{LiOH}$ followed by TBTU mediated amidation provided $\mathbf{2 2}$ and $\mathbf{2 3}$ in high yields (Scheme 5). Based on compounds $\mathbf{2 1}, \mathbf{2 2}$, and $\mathbf{2 3}$, one of three different derivatisation pathways were used to access final screening compounds. Scheme 6 shows one example of each reaction sequence. To further demonstrate functional group tolerability, additional compounds were synthesised using these three routes with varying $\mathrm{N}$-functionalisations. An overview of these compounds are shown in Scheme 7.

From compound $\mathbf{2 1}$, both $\mathrm{N}$-functionalisations were performed in the same reaction sequence (Scheme 6). First, piperidine derivatisation was achieved by removal of the Boc-group with TFA followed by reductive alkylation. Then, benzyl deprotection and 


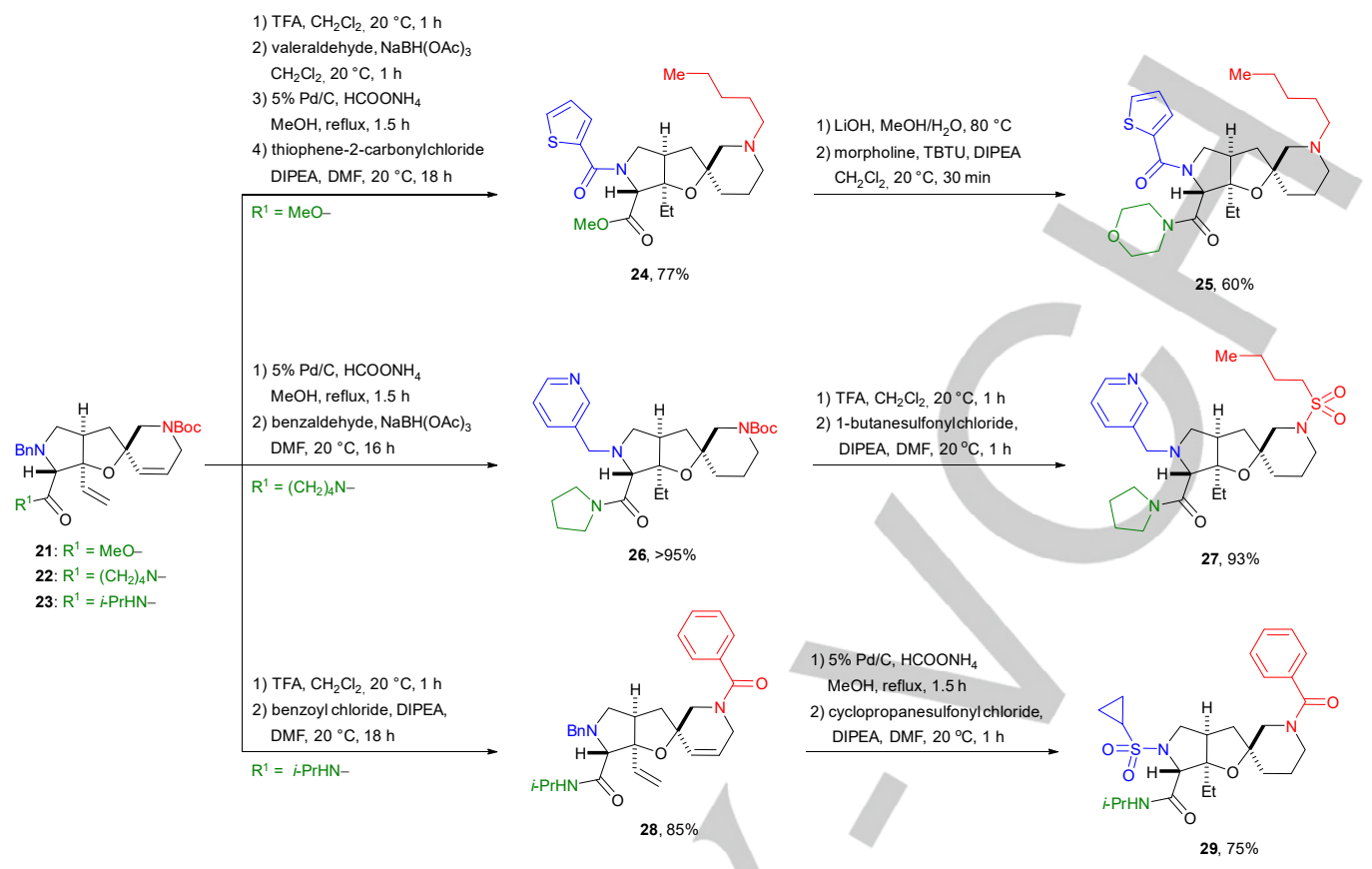

Scheme 6: Library $\mathrm{C}$ scaffold diversification through ester amidation and $\mathrm{N}$-functionalization of pyrrolidine and piperidine rings. Orthogonal protecting groups allowed for variation in the order of functionalizations which is exemplified by three different routes. All compounds in library $\mathrm{C}$ were synthesised using one of these three overall derivatisation pathways.

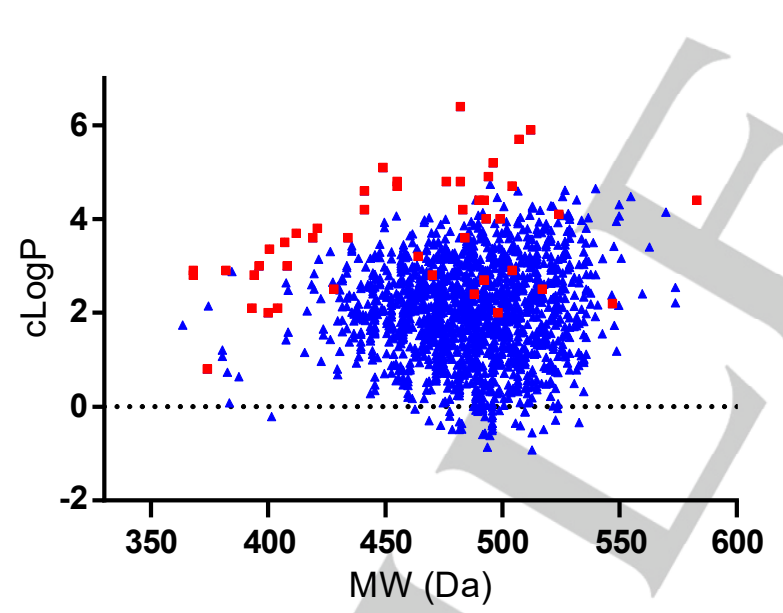

Figure 2: Physical chemical property analysis of the compounds reported here (red) compared to entire library of 2,188 high-throughput screening compounds synthesised for the European Lead Factory (blue).

\section{Conclusions}

In conclusion, we were able to construct a compact functionalized scaffold as a single diastereomer in one step from allylic amines, furylboronic acids, and $\alpha$-hydroxylated aldehydes by employing a highly diastereoselective 3-component Petasis/Diels-Alder tandem reaction. By simply varying the $\mathrm{N}$-substituent on the boronic acid building block or the $\mathrm{R}^{3}$ substituent on the aldehyde, we could access the core structures for libraries A-C. By judicious selection of the protecting group strategy in the initial step, we executed the synthesis of the libraries with a wide variety of different functional groups, thus providing a method to access an expansive set of natural product-like and $\mathrm{sp}^{3}$-rich compounds for biological testing. 2,188 of these have been included in the ELF Joint European Compounds Library.

\section{Experimental Section}

All reagents and solvents were purchased from commercial suppliers and used without further purification. All solvents used were of HPLC-grade, which predominantly were used without further drying. Unless otherwise stated, reactions were run as open-system reactions, using only a loosely fitted plastic plug in order to avoid contamination of the reaction mixture. Reaction products have been purified using flash column chromatography or preparative high-performance liquid chromatography (prepHPLC). Reactions were routinely monitored using thin layer chromatography (TLC), ultra-performance liquid-chromatography mass-spectrometry (UPLC-MS) and/or high performance liquidchromatography with UV detection (HPLC-UV). Analytical TLC was performed using Merck aluminum sheets covered with silica (C60). The plates were visualized using UV light and/or a $\mathrm{KMnO}_{4}$ staining solution $\left(3 \mathrm{~g}\right.$ in water $(300 \mathrm{~mL}), \mathrm{K}_{2} \mathrm{CO}_{3}(20 \mathrm{~g})$ and $5 \%$ aq $\mathrm{NaOH}(5 \mathrm{~mL}))$ followed by heating. Analytical UPLC-MS (ESI) 
was performed on a S2 Waters ACQUITY RPUPLC system equipped with a diode array detector using an ACQUITY UPLC BEH C18 column (d $1.7 \mu \mathrm{m}, 2.1 \times 50 \mathrm{~mm}$; column temp: $65^{\circ} \mathrm{C}$; flow: $0.6 \mathrm{~mL} / \mathrm{min}$ ), as well as a SQD ESI MS detector. Eluents $\mathrm{A} 1$ $\left(0.1 \% \mathrm{HCOOH}\right.$ in $\left.\mathrm{H}_{2} \mathrm{O}\right), \mathrm{A} 2\left(0.1 \% \mathrm{NH}_{4} \mathrm{COOCH}_{3}\right), \mathrm{B} 1(0.1 \%$ $\mathrm{HCOOH}$ in $\mathrm{MeCN})$ and $\mathrm{B} 2\left(0.1 \% \mathrm{NH}_{4} \mathrm{COOCH}_{3}\right.$ in $\left.\mathrm{MeCN}\right)$ were used in a linear gradient $5 \% \mathrm{~B} 1 / \mathrm{B} 2$ to $100 \% \mathrm{~B} 1 / \mathrm{B} 2$ in a total run time of $2.6 \mathrm{~min}$. Analytical LC-HRMS (ESI) analysis was performed on an Agilent 1100 RP-LC system equipped with a diode array detector using a Phenomenex Luna C18 column (d 3 $\mu \mathrm{m}, 2.1 \times 50 \mathrm{~mm}$; column temp: $40^{\circ} \mathrm{C}$; flow: $0.4 \mathrm{~mL} / \mathrm{min}$ ).
Eluents $\mathrm{A}\left(0.1 \% \mathrm{HCOOH}\right.$ in $\left.\mathrm{H}_{2} \mathrm{O}\right)$ and $\mathrm{B}(0.1 \% \mathrm{HCOOH}$ in $\mathrm{MeCN})$ were used in a linear gradient $(20 \% \mathrm{~B}$ to $100 \% \mathrm{~B})$ in a total run time of $15 \mathrm{~min}$. The LC system was coupled to a Micromass LCT orthogonal time-of-flight mass spectrometer equipped with a Lock Mass probe operating in positive electrospray mode. Flash column chromatography was achieved using a glass column packed with Merck Geduran® 60 silica gel (40-63 $\mu$ m particles) as stationary phase, and liquid phase as specified in the individual experimental.
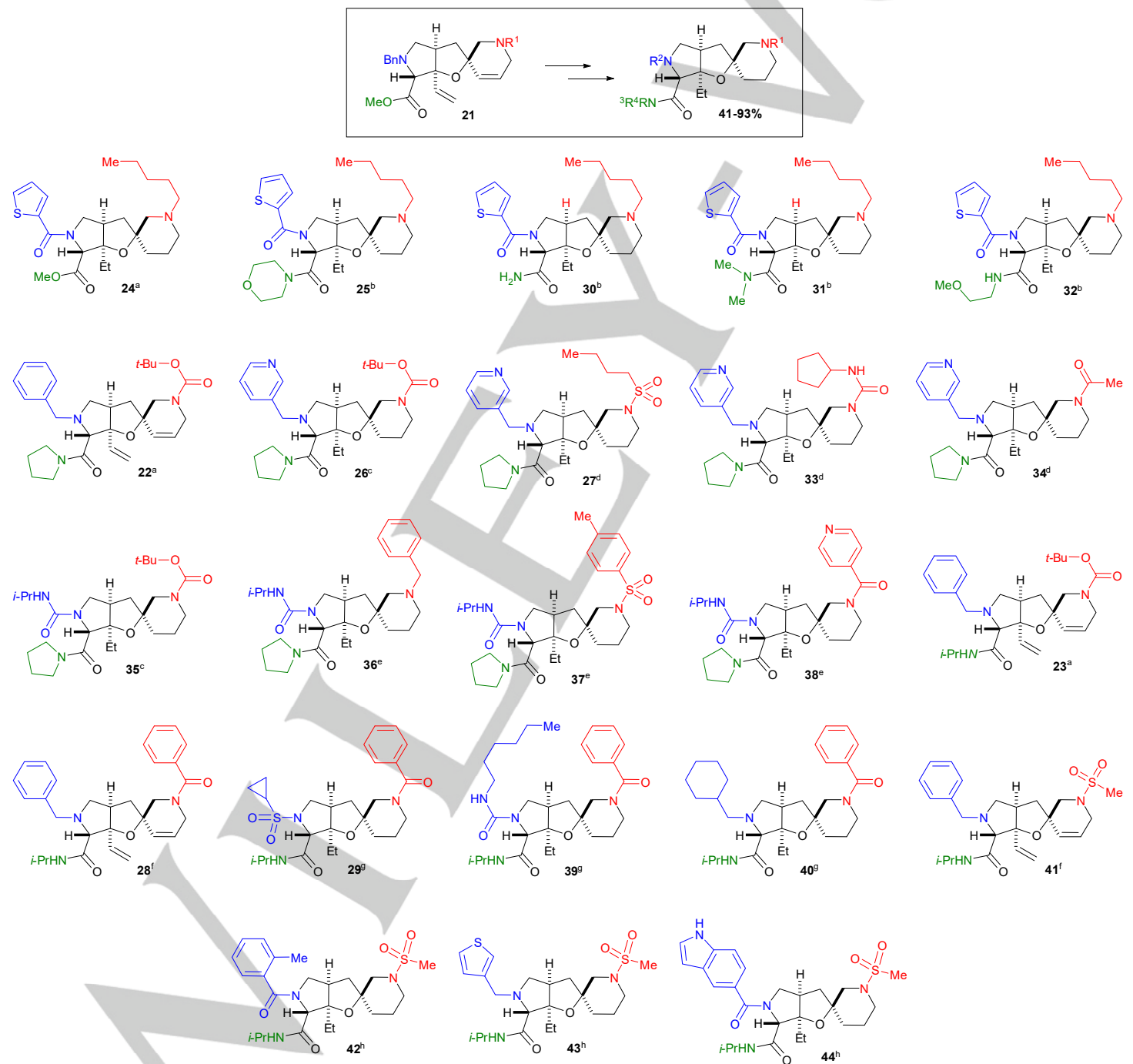

Scheme 7: Functional group tolerability in functionalization of library C. From 21, 23 screening compounds were synthesised with varying $R^{1}-R^{5}$ substituents. Compounds were synthesised using one of the three derivatisation pathways depicted in Scheme 6 . Yields of final compounds were 41$93 \%$ over 2 steps. See appendix for reagents and conditions. a synthesised from 21; b ${ }^{2}$ synthesised from 24 ; ${ }^{c}$ synthesised from 22 ; ${ }^{d}$ synthesised from 22 ; ${ }^{\mathrm{e}}$ synthesised from $35 ;{ }^{f}$ synthesised from $23 ;{ }^{9}$ synthesised from $28 ;{ }^{\mathrm{h}}$ synthesised from 41. 
All purified compounds have been routinely characterized by ${ }^{1} \mathrm{H}$ NMR, ${ }^{13} \mathrm{C}$ NMR, RP-UPLC-MS, and RP-HPLC-UV. Novel compounds were further characterized via HRMS. NMR spectra were recorded on a Bruker Ascend spectrometer with a Prodigy cryoprobe (operating at $400 \mathrm{MHz}$ for ${ }^{1} \mathrm{H}$ NMR and at $101 \mathrm{MHz}$ for ${ }^{13} \mathrm{C} N M R$ ), and analyzed via the NMR software MestReNova (version 6.2.1-7569) released by Mestrelab Research S.L. The chemical shifts $(\delta)$ are reported in parts per million $(\mathrm{ppm})$ and the coupling constants $(J)$ in $\mathrm{Hz}$. The majority of the spectra have been recorded in $\mathrm{CDCl}_{3}$, and the signals were adjusted relative to this position ( $\delta 7.26 \mathrm{ppm}$ for ${ }^{1} \mathrm{H}$ NMR and $\delta 77.2 \mathrm{ppm}$ for ${ }^{13} \mathrm{C} N M R$ ). For spectra recorded in DMSO-d6, the signals were adjusted relative to the DMSO signal $\left(\delta 2.5 \mathrm{ppm}\right.$ for ${ }^{1} \mathrm{H}$ NMR and $\delta 39.5$ ppm for ${ }^{13} \mathrm{C}$ NMR). For spectra recorded in $\mathrm{CD}_{3} \mathrm{OD}$, signal were adjusted relative to this position $\left(\delta 3.31 \mathrm{ppm}\right.$ for ${ }^{1} \mathrm{H}$ NMR and $\delta$ $49.00 \mathrm{ppm}$ for ${ }^{13} \mathrm{C}$ NMR).Compounds have been drawn and named through use of the visualization software ChemDraw Ultra 14.0 released by PerkinElmer Informatics. Molecular- and exact masses have been calculated via this program as well.

\section{General Method A. Mitsunobu conditions for arylation of alcohols}

Alcohol (0.19 mmol), 5-hydroxy-2-methylpyridine (25 mg, 0.23 $\mathrm{mmol}$ ) and triphenylphosphine $(60 \mathrm{mg}, 0.23 \mathrm{mmol})$ was dissolved in dry THF ( $2 \mathrm{~mL}, 0.1 \mathrm{M})$ under nitrogen atmosphere and cooled to $0{ }^{\circ} \mathrm{C}$.DEAD ( $131 \mu \mathrm{L}$ of a $40 \%$ toluene solution, $0.29 \mathrm{mmol}$ ) was added dropwise and then warmed slowly to $20^{\circ} \mathrm{C}$ where it was stirred for 16 hours. The reaction was concentrated, and the crude was purified by flash column chromatography.

General Method B. Hydrogenation conditions for deprotection of benzyl groups and/or for the reduction of olefins

Benzylamine or olefin $(2.56 \mathrm{mmol})$ was dissolved in $\mathrm{MeOH}(9 \mathrm{~mL}$, $0.3 \mathrm{M})$ and added $\mathrm{Pd} / \mathrm{C}(5 \mathrm{wt} \% \mathrm{Pd} / \mathrm{C}(10 \%), 0.13 \mathrm{mmol})$ and $\mathrm{HCOONH}_{4}(12.8 \mathrm{mmol}, 5$ equiv.) and the mixture was stirred 1.5 $h$ at reflux. The reaction mixture was then filtered using celite and the filtrate was concentrated in vacuo. The residue was added $\mathrm{CH}_{2} \mathrm{Cl}_{2}(30 \mathrm{~mL}), \mathrm{H}_{2} \mathrm{O}(20 \mathrm{~mL})$ and sat. $\mathrm{NaHCO}_{3}$ (aq.) $(10 \mathrm{~mL})$ and the layers were separated. The aqueous phase was extracted with $\mathrm{CH}_{2} \mathrm{Cl}_{2}(2 \times 30 \mathrm{~mL})$ and the combined organic layers were dried over $\mathrm{Na}_{2} \mathrm{SO}_{4}$ and concentrated in vacuo to give the reduced compound.

\section{General Method C. Hydrogenation conditions for} deprotection of benzyl groups and/or for the reduction of olefins

Benzyl amine or olefin $(2.05 \mathrm{mmol})$ was dissolved in $\mathrm{EtOH}: \mathrm{H}_{2} \mathrm{O}$ $(10: 1,20 \mathrm{~mL}, 0.1 \mathrm{M})$. Pd/C (109 mg of $10 \% \mathrm{Pd} / \mathrm{C}, 0.103 \mathrm{mmol})$ was added and stirred at $60^{\circ} \mathrm{C}$ under a hydrogen gas atmosphere for 28 hours. The reaction mixture was filtered through a celite pad and the filtrate was concentrated.

General Method D. Reductive amination conditions
Amine $(0.53 \mathrm{mmol})$ was dissolved in $\mathrm{MeOH}(5 \mathrm{~mL}, 0.1 \mathrm{M})$. Aldehyde $(0.79 \mathrm{mmol}), \mathrm{AcOH}(45 \mu \mathrm{L}, 0.79 \mathrm{mmol})$, and sodium cyanoborohydride $(50 \mathrm{mg}, 0.79 \mathrm{mmol}$ ) were added to the solution. The reaction was stirred for 16 hours at $20^{\circ} \mathrm{C}$. The reaction was concentrated and partitioned between sat. aq. $\mathrm{NaHCO}_{3}(25 \mathrm{~mL})$ and $\mathrm{CH}_{2} \mathrm{Cl}_{2}(25 \mathrm{~mL})$. The two phases were separated and the aqueous phase extracted with $\mathrm{CH}_{2} \mathrm{Cl}_{2}(2 \times 25 \mathrm{~mL})$. The combined organic phases were dried over sodium sulfate anhydrous, filtered and concentrated. The crude was purified by flash column chromatography to provide the alkylated amine.

\section{General Method E. Reductive amination conditions}

Amine $(1.39 \mathrm{mmol})$ was dissolved in $\mathrm{CH}_{2} \mathrm{Cl}_{2}(7 \mathrm{~mL})$. Aldehyde (1.74 mmol, 1.25 equiv.) was added followed by $\mathrm{NaBH}(\mathrm{OAc})_{3}$ (426 mg, $2.01 \mathrm{mmol}, 1.5$ equiv.). The mixture was stirred $1 \mathrm{~h}$ at $20{ }^{\circ} \mathrm{C}$ and then purified directly using flash column chromatography.

\section{General Method F. Boc deprotection of amines}

Boc protected amine $(0.19 \mathrm{mmol})$ was dissolved in a 3:1 mixture of DCM and TFA $(0.1 \mathrm{M}, 1.5 \mathrm{~mL})$. The reaction was stirred for $1 \mathrm{~h}$ at $20{ }^{\circ} \mathrm{C}$ and then concentrated under reduced pressure. The crude was solubilized in ethyl acetate $(20 \mathrm{~mL})$ and washed with sat. aq. $\mathrm{NaHCO}_{3}(2 \times 15 \mathrm{~mL})$, dried over sodium sulfate anhydrous, filtered and concentrated. The crude was used directly in the next step without further purification.

\section{General Method G. Acylation of amines using isocyanate}

Amine $(0.05 \mathrm{mmol})$ was dissolved in $1 \mathrm{~mL}$ of DMF. Isopropyl isocyanate $(0.06 \mathrm{mmol})$ was added and stirred $18 \mathrm{~h}$ at $20^{\circ} \mathrm{C}$. The reaction mixture was diluted with $1 \mathrm{~mL}$ of DMF and purified by preparative HPLC to give the desired acylated amine.

\section{General Method H. Acylation of amines using acid chlorides}

Amine $(0.07 \mathrm{mmol})$ was dissolved in $1 \mathrm{~mL}$ of DMF. DIPEA (36 $\mu \mathrm{L}$, $0.21 \mathrm{mmol})$ and acid chloride $(0.077 \mathrm{mmol})$ was added, and the reaction stirred $18 \mathrm{~h}$ at $20^{\circ} \mathrm{C}$. The reaction mixture was diluted with $1 \mathrm{~mL}$ of DMF and purified by preparative HPLC to give the desired compound.

\section{General Method I. Acylation of amines using acid chlorides}

Methyl ester $(6.96 \mathrm{mmol})$ was dissolved in $\mathrm{MeOH}: \mathrm{H}_{2} \mathrm{O}(24 \mathrm{~mL}$, 2:1) and added $\mathrm{LiOH}(500 \mathrm{mg}, 20.9 \mathrm{mmol}, 3$ equiv.) and the mixture was stirred $5 \mathrm{~h}$ at $80^{\circ} \mathrm{C}$. The mixture was cooled to $20^{\circ} \mathrm{C}$ and added $\mathrm{H}_{2} \mathrm{O}(40 \mathrm{~mL})$ and ether $(30 \mathrm{~mL})$ and the layers were separated. The organic layer was extracted with $1 \mathrm{M} \mathrm{NaOH}(20$ $\mathrm{mL}$ ) and the combined aqueous phases were combined and acidified $(\mathrm{pH}=6)$. The aqueous phase was then extracted with $\mathrm{CH}_{2} \mathrm{Cl}_{2}(3 \times 60 \mathrm{~mL})$ and the combined organic layers were dried over $\mathrm{Na}_{2} \mathrm{SO}_{4}$ and concentrated in vacuo. The crude extract was used in the next step without further purification.

\section{General Method J. Amide formation from carboxylic acids}

Carboxylic acid $(3.47 \mathrm{mmol})$ was dissolved in $\mathrm{CH}_{2} \mathrm{Cl}_{2}(11 \mathrm{~mL}, 0.3$ $\mathrm{M})$ and added DIPEA (0.91 mL, $5.21 \mathrm{mmol}, 1.5$ equiv.) followed by TBTU (1.23 g, $3.82 \mathrm{mmol}, 1.1$ equiv.) and the mixture was stirred at $20{ }^{\circ} \mathrm{C}$. After $5 \mathrm{~min}$, amine $(6.96 \mathrm{mmol}$, 2 equiv) was added and the mixture was stirred for another $30 \mathrm{~min}$. The mixture 
was then concentrated and purified by flash column chromatography to provide the desired amide product.

\section{General Method K. Sulfonylation of amines}

Amine $(1.49 \mathrm{mmol})$ was dissolved in $\mathrm{CH}_{2} \mathrm{Cl}_{2}(8 \mathrm{~mL})$, added $\mathrm{Et}_{3} \mathrm{~N}$ (312 $\mu \mathrm{L}, 2.24 \mathrm{mmol}, 1.5$ equiv.) and cooled to $0{ }^{\circ} \mathrm{C}$. The mixture was added sulfonyl chloride ( $1.79 \mathrm{mmol}, 1.2$ equiv.), and the mixture was stirred $1 \mathrm{~h}$ at $0{ }^{\circ} \mathrm{C}$ and then purified directly using flash column chromatography to give the desired sulfonylated amine.

\section{Synthesis of building blocks for library A-C}

\section{Synthesis of $N$-benzylprop-2-en-1-amine}

N-Benzylprop-2-en-1-amine (S1). Benzaldehyde $(40 \mathrm{~g}, 38.5 \mathrm{~mL}$, $0.377 \mathrm{~mol})$ was dissolved in $\mathrm{MeOH}(300 \mathrm{~mL}, 1.2 \mathrm{M})$ and allyl amine was added $(43 \mathrm{~g}, 57 \mathrm{~mL}, 0.753 \mathrm{~mol}$ ) (Warning: Exothermic reaction). After stirring for $5 \mathrm{~min}, \mathrm{MgSO}_{4}(40 \mathrm{~g})$ was added and the mixture was stirred $1 \mathrm{~h}$ at $20^{\circ} \mathrm{C}$. The mixture was then cooled to $0{ }^{\circ} \mathrm{C}$ and added $\mathrm{NaBH}_{4}(15.7 \mathrm{~g}, 0.415 \mathrm{mmol})$ portion wise over 30 min (Warning: Very exothermic reaction) and after the last addition the mixture was stirred for another $30 \mathrm{~min}$. The ice bath was then removed and the mixture was stirred $1 \mathrm{~h}$ at $20^{\circ} \mathrm{C}$. where upon sat. aq. $\mathrm{NaHCO}_{3}(100 \mathrm{~mL})$ was added and the mixture stirred for more $30 \mathrm{~min}$. The mixture was then filtered and the volatiles were removed under reduced pressure. The residue was partitioned between water $(100 \mathrm{~mL})$ and EtOAc $(200 \mathrm{~mL})$ and the layers separated. The aqueous phase was extracted with EtOAc $(100 \mathrm{~mL})$ and the combined organic layers were washed with brine, dried over sodium sulfate, filtered and concentrated in vacuo to give the title compound as a colorless oil (49 $\mathrm{g}, 88 \%)$ that was used directly in the next step without further purification.

\section{Synthesis of boronic acid building blocks}

(5-(((Tert-butoxycarbonyl)(methyl)amino)methyl)furan-2-

yl)boronic acid (3). (5-Formylfuran-2-yl)boronic acid (2) $(2.04 \mathrm{~g}$, $14.6 \mathrm{mmol})$ and methyl amine (33\% in ethanol) $(3.6 \mathrm{~mL}, 29.0$ $\mathrm{mmol})$ were dissolved in $\mathrm{MeOH}(36 \mathrm{~mL}, 0.4 \mathrm{M})$ and $3 \AA$ molecular sieves $(3 \mathrm{~g})$ were added. The mixture was stirred $1.5 \mathrm{~h}$ at $20^{\circ} \mathrm{C}$, where after the mixture was cooled to $0{ }^{\circ} \mathrm{C}$ in an ice bath and added $\mathrm{NaBH}_{4}(0.607 \mathrm{~g}, 16 \mathrm{mmol})$ portion wise. After the addition, the mixture was stirred for $10 \mathrm{~min}$ at $0^{\circ} \mathrm{C}$ followed by $1 \mathrm{~h}$ at $20^{\circ} \mathrm{C}$. The reaction mixture was concentrated in vacuo and the crude mixture used directly in the next step.

The crude was dissolved in $\mathrm{CH}_{2} \mathrm{Cl}_{2}(36 \mathrm{~mL}, 0.4 \mathrm{M})$, added $\mathrm{Et}_{3} \mathrm{~N}(6$ $\mathrm{mL}, 43.7 \mathrm{mmol}, 3$ equiv.) followed by $\mathrm{Boc}_{2} \mathrm{O}(3.2 \mathrm{~g}, 14.6 \mathrm{mmol})$ dissolved in $\mathrm{CH}_{2} \mathrm{Cl}_{2}(8 \mathrm{~mL})$ and the mixture was stirred 30 min at $20{ }^{\circ} \mathrm{C}$. Then sat. aq. $\mathrm{NaHCO}_{3}(50 \mathrm{~mL})$ was added and the layers separated. The aqueous phase was extracted with $\mathrm{CH}_{2} \mathrm{Cl}_{2}(2 \times 50$ $\mathrm{mL}$ ) and the combined organic layers were dried over sodium sulfate anhydrous, filtered and concentrated. The crude was used directly in without further purification.

(5-((Allyl(tert-butoxycarbonyl)amino)methyl)furan-2-yl)boronic acid (4). (5-Formylfuran-2-yl)boronic acid $(10 \mathrm{~g}, 71 \mathrm{mmol})$ and allyl amine $(16 \mathrm{~mL}, 214 \mathrm{mmol})$ were dissolved in $\mathrm{MeOH}(180 \mathrm{~mL}$, $0.4 \mathrm{M})$ and $3 \AA$ molecular sieves were added, and the mixture stirred $1 \mathrm{~h}$ at $20^{\circ} \mathrm{C}$. Then, the mixture was cooled to $0{ }^{\circ} \mathrm{C}$ in an ice bath and added $\mathrm{NaBH}_{4}(3.0 \mathrm{~g}, 79 \mathrm{mmol})$ portion wise. The mixture was dissolved in $\mathrm{CH}_{2} \mathrm{Cl}_{2}(50 \mathrm{~mL})$ and $\mathrm{Et}_{3} \mathrm{~N}(5 \mathrm{~mL})$ and the mixture was again concentrated in vacuo. (NOTE: done to ensure full removal of allyl amine before step 2).

The crude was dissolved in $\mathrm{CH}_{2} \mathrm{Cl}_{2}(150 \mathrm{~mL}, 0.4 \mathrm{M})$, added $\mathrm{Et}_{3} \mathrm{~N}$ (20 mL, $143 \mathrm{mmol}, 2$ equiv.) followed by $\mathrm{Boc}_{2} \mathrm{O}(15.6 \mathrm{~g}, 71 \mathrm{mmol})$ dissolved in $\mathrm{CH}_{2} \mathrm{Cl}_{2}(30 \mathrm{~mL})$ and the mixture was stirred $1 \mathrm{~h}$ at $20{ }^{\circ} \mathrm{C}$. Then sat. aq. $\mathrm{NaHCO}_{3}(200 \mathrm{~mL})$ was added and the layers were separated. The aqueous phase was extracted with $\mathrm{CH}_{2} \mathrm{Cl}_{2}$ $(2 \times 100 \mathrm{~mL})$ and the combined organic layers were dried over sodium sulfate anhydrous, concentrated to $100 \mathrm{~mL}$ and used directly in the next step without further purification.

\section{Synthesis of aldehyde building block}

2-Hydroxypent-4-enoic acid 5 (S2). To a solution of allyl bromide $(11.3 \mathrm{~mL}, 131 \mathrm{mmol})$ in THF: $\mathrm{H}_{2} \mathrm{O}(2: 1)(300 \mathrm{~mL})$ at $0{ }^{\circ} \mathrm{C}$ was added glyoxylic acid $(8 \mathrm{~g}, 87 \mathrm{mmol})$. Then, indium (11 g, $96 \mathrm{mmol})$ was added in one portion and the suspension stirred vigorously, and allowed to reach $20^{\circ} \mathrm{C}$, where it was stirred for more $19 \mathrm{~h}$. The reaction was quenched by the addition of $\mathrm{HCl} 1 \mathrm{M}(350 \mathrm{~mL})$ and the aqueous phase was extracted with $\mathrm{CH}_{2} \mathrm{Cl}_{2}(3 \times 350$ $\mathrm{mL}$ ). The combined organic layers were dried over sodium sulfate anhydrous, filtered and concentrated. The crude was distilled under reduced pressure $\left(2.7 \mathrm{mbar}, 120-130{ }^{\circ} \mathrm{C}\right)$, to give the title compound as a colorless oil $(4.85 \mathrm{~g}, 48 \%) .{ }^{1} \mathrm{H}$ NMR $(400 \mathrm{MHz}$, $\left.\mathrm{CDCl}_{3}\right) \delta 6.12$ (broad s, $\left.1 \mathrm{H}\right), 5.88-5.76(\mathrm{~m}, 1 \mathrm{H}), 5.25-5.13(\mathrm{~m}$, $2 \mathrm{H}), 4.36(\mathrm{dd}, J=6.7,4.5 \mathrm{~Hz}, 1 \mathrm{H}), 2.70-2.58(\mathrm{~m}, 1 \mathrm{H}), 2.56-$ $2.43(\mathrm{~m}, 1 \mathrm{H}) .{ }^{13} \mathrm{C}$ NMR $\left(101 \mathrm{MHz}, \mathrm{CDCl}_{3}\right) \delta 178.7,132.1,119.6$, $69.9,38.5$. The data is in accordance with the literature [ACS Comb. Sci. 2012, 14, 253-257].

5-Allyl-2,2-dimethyl-1,3-dioxolan-4-one (S3). To a solution of 2hydroxypent-4-enoic acid (S4) $(4.85 \mathrm{~g}, 41.8 \mathrm{mmol})$ in acetone (84 $\mathrm{mL}$ ) was added 2,2-dimethoxypropane $(41.1 \mathrm{~mL}, 334 \mathrm{mmol})$ and pyridinium p-toluenesulfonate $(5.25 \mathrm{~g}, 20.9 \mathrm{mmol})$ and the reaction stirred at reflux for $2 \frac{1}{2} \mathrm{~h}$. The reaction was allowed to cool to $20{ }^{\circ} \mathrm{C}$ and filtered through a pad of celite, and the solid washed with EtOAc $(2 \times 100 \mathrm{~mL})$. The filtrate was concentrated and the crude distilled $\left(3-4 \mathrm{mbar}, 80^{\circ} \mathrm{C}\right)$, to give the title compound as colorless oil $(5.3 \mathrm{~g} / 33.9 \mathrm{mmol}) .{ }^{1} \mathrm{H}$ NMR $(400 \mathrm{MHz}$, $\mathrm{CDCl}_{3}$ ) $\delta 5.79$ (ddt, $\left.J=17.1,10.2,6.9 \mathrm{~Hz}, 1 \mathrm{H}\right), 5.22-5.12(\mathrm{~m}$, $2 \mathrm{H}$ ), 4.45 (dd, $J=6.7,4.4 \mathrm{~Hz}, 1 \mathrm{H}$ ), 2.62 (dddt, $\mathrm{J}=14.8,6.9,4.4$, $1.4 \mathrm{~Hz}, 1 \mathrm{H}), 2.46$ (dtt, $\mathrm{J}=14.9,6.9,1.3 \mathrm{~Hz}, 1 \mathrm{H}), 1.58(\mathrm{~d}, \mathrm{~J}=0.8$ $\mathrm{Hz}, 3 \mathrm{H}), 1.52(\mathrm{~d}, \mathrm{~J}=0.8 \mathrm{~Hz}, 3 \mathrm{H}) .{ }^{13} \mathrm{C} \mathrm{NMR}\left(101 \mathrm{MHz}, \mathrm{CDCl}_{3}\right) \delta$ $172.6,131.9,119.2,110.7,73.8,35.8,27.2,26.0$. The data is in accordance with the literature [ACS Comb. Sci. 2012, 14, 253-257].

5-Allyl-2,2-dimethyl-1,3-dioxolan-4-ol (S4). To a solution of 5-allyl2,2-dimethyl-1,3-dioxolan-4-one (2.55 g, $16.3 \mathrm{mmol})$ (S3) in dry toluene $(25 \mathrm{~mL})$ at $-78^{\circ} \mathrm{C}$ was added dropwise DIBAL-H $(24.4 \mathrm{~mL}$ of a $1 \mathrm{M}$ DIBAL-H toluene solution, $24.4 \mathrm{mmoL}$ ) and after $30 \mathrm{~min}$, the reaction was quenched with aqueous $1 \mathrm{M} \mathrm{HCl}(30 \mathrm{~mL})$. The reaction was allowed to warm to $20^{\circ} \mathrm{C}$ and stirred for another 30 min, whereupon the reaction mixture was diluted with $\mathrm{H}_{2} \mathrm{O}(100$ $\mathrm{mL})$ and extracted with $\mathrm{CH}_{2} \mathrm{Cl}_{2}(3 \times 100 \mathrm{~mL})$. The combined organic layers were dried over sodium sulfate anhydrous and concentrated to give the title compound as colorless oil which was used directly in the next step without any purification.

\section{Petasis/Diels-Alder Tandem Reaction in the Synthesis of Library A-C Scaffold and Library Production}

Tert-butyl $\quad\left(\left(\left(3 S^{*}, 3 a R^{*}, 6 R^{*}, 7 a R^{*}\right)-2-\right.\right.$-benzyl-3-(hydroxymethyl) 2,3,7,7a-tetrahydro-3a,6-epoxyisoindol-6(1H)- 
yl)methyl)(methyl)carbamate (5). To a solution containing glycolaldehyde dimer $(700 \mathrm{mg}, 5.85 \mathrm{mmol})$ and $\mathrm{N}$ allylbenzylamine (S1) $(2.15 \mathrm{~mL}, 14.6 \mathrm{mmol})$ dissolved in $\mathrm{CH}_{2} \mathrm{Cl}_{2}$ $(36 \mathrm{~mL}, \quad 0.4 \mathrm{M})$ was added the crude (5-(((tertbutoxycarbonyl)(methyl)amino)methyl)furan-2-yl)boronic acid (3)

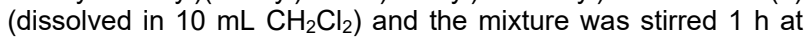
$20{ }^{\circ} \mathrm{C}$. Then, sat. aq. $\mathrm{NaHCO}_{3}(50 \mathrm{~mL})$ was added and the layers were separated. The aqueous phase was extracted with $\mathrm{CH}_{2} \mathrm{Cl}_{2}$ $(2 \times 50 \mathrm{~mL})$ and the combined organic layers were dried over sodium sulfate anhydrous, filtered and concentrated. The crude was taken in MeCN ( $36 \mathrm{~mL}, 0.4 \mathrm{M}$ ) and the mixture was stirred at reflux for $24 \mathrm{~h}$. The mixture was then concentrated and purified by flash column chromatography (EtOAc:heptane 1:1, $R_{\mathrm{f}}=0.2$ ) to give the title compound as a yellow oil $(3.502 \mathrm{~g}, 75 \%) .{ }^{1} \mathrm{H}$ NMR $\left(400 \mathrm{MHz}, \mathrm{CDCl}_{3}\right) \delta 7.31-7.12(\mathrm{~m}, 5 \mathrm{H}), 6.49(\mathrm{~d}, \mathrm{~J}=5.8 \mathrm{~Hz}, 1 \mathrm{H})$, $6.18(\mathrm{~d}, \mathrm{~J}=5.8 \mathrm{~Hz}, 1 \mathrm{H}), 3.93(\mathrm{~d}, \mathrm{~J}=13.7 \mathrm{~Hz}, 2 \mathrm{H}$, major rotamer), $3.74(\mathrm{~d}, \mathrm{~J}=11.4 \mathrm{~Hz}, 1 \mathrm{H}), 3.70-3.54(\mathrm{~m}, 3 \mathrm{H}), 3.48(\mathrm{~d}, \mathrm{~J}=13.7$ $\mathrm{Hz}, 2 \mathrm{H}$, minor rotamer), $3.17(\mathrm{t}, \mathrm{J}=7.7 \mathrm{~Hz}, 1 \mathrm{H}), 2.96(\mathrm{~s}, 1 \mathrm{H}), 2.86$ (s, 3H), 2.17 (dd, $J=10.6,8.7 \mathrm{~Hz}, 1 \mathrm{H}$ ), 1.91 (dtd, $J=10.3,6.9$, 6.5, $2.9 \mathrm{~Hz}, 1 \mathrm{H}), 1.41(\mathrm{~s}, 9 \mathrm{H}), 1.40-1.22(\mathrm{~m}, 2 \mathrm{H}) .{ }^{13} \mathrm{C}$ NMR $(101$ $\left.\mathrm{MHz}, \mathrm{CDCl}_{3}\right) \delta 156.4$ (major rotamer), 155.7 (minor rotamer), 138.4 (major rotamer), 138.4 (minor rotamer), 136.9 (major rotamer), 136.6 (minor rotamer), 136.4 (major rotamer), 136.1 (minor rotamer), 129.0, 128.6 (2 carbons), 127.5 ( 2 carbons), 98.9 92.4 (major rotamer), 91.8 (minor rotamer), 79.9 (minor rotamer), 79.6 (major rotamer), 65.8 (major rotamer), 65.7 (minor rotamer), 59.7, 58.7, 58.5, 50.6 (minor rotamer), 49.8 (major rotamer), 45.2, 36.0 (major rotamer), 35.9 (minor rotamer), 32.6 (major rotamer), 32.5 (minor rotamer), 28.5 (3C). HRMS (ESI) calcd for $\mathrm{C}_{23} \mathrm{H}_{33} \mathrm{~N}_{2} \mathrm{O}_{4}\left[\mathrm{M}+\mathrm{H}^{+}\right]$401.2435, found 401.2468.

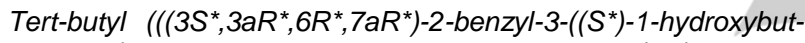
3-en-1-yl)-2,3,7,7a-tetrahydro-3a,6-epoxyisoindol-6(1H)-

yl)methyl)(methyl)carbamate (6). To a solution of 5-allyl-2,2dimethyl-1,3-dioxolan-4-ol (S4) (1.1g, $7.0 \mathrm{mmol})$ and $\mathrm{N}$ allylbenzylamine (S1) $(0.93 \mathrm{~g}, 6.3 \mathrm{mmol})$ in $\mathrm{CH}_{2} \mathrm{Cl}_{2}(36 \mathrm{~mL}, 0.4 \mathrm{M})$ was added (5-((tert-butoxycarbonyl)(methyl)amino)methyl)furan2-yl)boronic acid (3) $(1.86 \mathrm{~g}, 7.3 \mathrm{mmol})$, dissolved in $10 \mathrm{~mL}$ $\mathrm{CH}_{2} \mathrm{Cl}_{2}$ ) and the mixture was stirred $1 \mathrm{~h}$ at $20^{\circ} \mathrm{C}$. Sat. aq. $\mathrm{NaHCO}_{3}$ $(50 \mathrm{~mL})$ was then added and the layers were separated. The aqueous phase was extracted with $\mathrm{CH}_{2} \mathrm{Cl}_{2}(2 \times 50 \mathrm{~mL})$ and the combined organic layers were dried over sodium sulfate anhydrous, filtered and concentrated. The crude was taken in acetonitrile $(18 \mathrm{~mL}, 0.4 \mathrm{M})$ and the mixture was stirred at reflux for $24 \mathrm{~h}$. The mixture was then concentrated and purified by flash column chromatography (EtOAc/heptane, 1:3, $R_{\mathrm{f}}=0.25$ ) to give the desired compound as a clear yellow oil $(0.68 \mathrm{~g}, 31 \%) .{ }^{1} \mathrm{H}$ NMR $\left(400 \mathrm{MHz}, \mathrm{CDCl}_{3}\right) \delta 7.37-7.25(\mathrm{~m}, 5 \mathrm{H}), 6.76(\mathrm{dd}, \mathrm{J}=10.2,5.7$ $\mathrm{Hz}, 1 \mathrm{H}), 6.13(\mathrm{dd}, \mathrm{J}=11.8,5.7 \mathrm{~Hz}, 1 \mathrm{H}), 5.97-5.79(\mathrm{~m}, 1 \mathrm{H}), 5.19$ $-5.06(\mathrm{~m}, 2 \mathrm{H}), 4.07-3.38(\mathrm{~m}, 5 \mathrm{H}), 3.16(\mathrm{br} . \mathrm{s}, 1 \mathrm{H}), 3.07-2.77$ $(\mathrm{m}, 5 \mathrm{H}$, two rotamers), $2.68-2.52(\mathrm{~m}, 1 \mathrm{H}), 2.51-2.32(\mathrm{~m}, 1 \mathrm{H})$, 2.11 (t, J = 9.6 Hz, 1H), 1.92 (br. s, 1H), 1.47 (s, 9H), $1.41-1.30$ (m, 2H). ${ }^{13} \mathrm{C}$ NMR $\left(101 \mathrm{MHz}, \mathrm{CDCl}_{3}\right) \delta 156.4$ (major rotamer), 155.8 (minor rotamer), 138.9, 137.4 (minor rotamer), 137.2 (major rotamer), 135.0, 134.8, 128.9, $128.6(2 \mathrm{C}), 127.4(2 \mathrm{C}), 117.6,97.6$, 91.6 (major rotamer), 91.1 (minor rotamer), 79.8 (major rotamer), 79.6 (minor rotamer), 69.7, 69.3, 58.7, 58.3, 50.5 (minor rotamer), 49.6 (major rotamer), 45.7, 37.0, 35.9, 32.5, 28.6 (3C). HRMS (ESI) calcd for $\mathrm{C}_{26} \mathrm{H}_{37} \mathrm{~N}_{2} \mathrm{O}_{4}\left[\mathrm{M}+\mathrm{H}^{+}\right]$441.2748, found 441.2770.

Methyl $\left(3 R^{\star}, 3 a R^{\star}, 6 R^{\star}, 7 a R^{\star}\right)-6-(($ allyl (tertbutoxycarbonyl)amino)methyl)-2-benzyl-1,2,3,6,7,7a-hexahydro3a,6-epoxyisoindole-3-carboxylate (7). Glyoxylic acid monohydrate $(6.6 \mathrm{~g}, 71 \mathrm{mmol})$ and $\mathrm{N}$-allylbenzylamine $(8.42 \mathrm{~mL}$, $57 \mathrm{mmol})$ was dissolved in $\mathrm{CH}_{2} \mathrm{Cl}_{2}(100 \mathrm{~mL})$. (5-((allyl(tertbutoxycarbonyl)amino)methyl)furan-2-yl)boronic acid (4) was added, and the mixture was stirred $18 \mathrm{~h}$ at $20^{\circ} \mathrm{C}$. (NOTE: the reaction usually takes around $1 \mathrm{~h})$. Water $(200 \mathrm{~mL})$ was added and the layers were separated. The aqueous phase was extracted with $\mathrm{CH}_{2} \mathrm{Cl}_{2}(2 \times 100 \mathrm{~mL})$, and the combined organic layers were dried over $\mathrm{Na}_{2} \mathrm{SO}_{4}$ and concentrated in vacuo. The crude was dissolved in acetone $(180 \mathrm{~mL}, 0.4 \mathrm{M})$, and $\mathrm{K}_{2} \mathrm{CO}_{3}(11.9 \mathrm{~g}, 86$ $\mathrm{mmol}$ ) was added followed by Mel $(4.45 \mathrm{~mL}, 71 \mathrm{mmol})$. The mixture was stirred for $4 \mathrm{~h}$ at $20^{\circ} \mathrm{C}$. Additional $\mathrm{K}_{2} \mathrm{CO}_{3}(5.5 \mathrm{~g}, 40$ $\mathrm{mmol})$ and $\mathrm{Mel}(2.3 \mathrm{~mL}, 37 \mathrm{mmol})$ were added, and the mixture was stirred $16 \mathrm{~h}$ at $20^{\circ} \mathrm{C}$. Then EtOAc $(200 \mathrm{~mL})$ was added and was washed with water. The aqueous phase was extracted with $\mathrm{CH}_{2} \mathrm{Cl}_{2}(2 \times 100 \mathrm{~mL})$, and the combined organic layers were dried over $\mathrm{Na}_{2} \mathrm{SO}_{4}$ and concentrated in vacuo. The residue was purified by silica plug (EtOAc:heptane $1: 4$ ) to give the methyl ester as a yellow oil. (NOTE: It is a lot easier to purify the final compound if some of the impurities are removed using the silica plug before the Diels-Alder reaction. Step 5: The compound from step 4 was taken in MeCN $(180 \mathrm{~mL})$ and the mixture was stirred at reflux for $24 \mathrm{~h}$. The mixture was then concentrated in vacuo and purified by flash column chromatography (EtOAc:heptane $3: 7, R_{\mathrm{f}}=0.2$ ) to give the title compound as a yellow oil $(11.5 \mathrm{~g}, 44 \%$ over 5 steps, based on allylbenzylamine). ${ }^{1} \mathrm{H}$ NMR $\left(400 \mathrm{MHz}, \mathrm{CDCl}_{3}\right) \delta 7.36-$ $7.10(\mathrm{~m}, 5 \mathrm{H}), 6.27-6.02(\mathrm{~m}, 2 \mathrm{H}), 5.79-5.58(\mathrm{~m}, 1 \mathrm{H}), 5.11-$ $4.94(\mathrm{~m}, 2 \mathrm{H}), 3.95-3.80(\mathrm{~m}, 2 \mathrm{H}), 3.81-3.61(\mathrm{~m}, 4 \mathrm{H}), 3.56(\mathrm{~s}$, $3 \mathrm{H}), 3.49(\mathrm{~s}, 1 \mathrm{H}), 3.51-3.42(\mathrm{~m}, 1 \mathrm{H}), 3.26(\mathrm{t}, \mathrm{J}=7.0 \mathrm{~Hz}, 1 \mathrm{H})$, $2.24-2.08(\mathrm{~m}, 2 \mathrm{H}), 1.38(\mathrm{~s}, 9 \mathrm{H}), 1.35-1.27(\mathrm{~m}, 1 \mathrm{H}) ;{ }^{13} \mathrm{C}$ NMR $\left(101 \mathrm{MHz}, \mathrm{CDCl}_{3}\right) \delta 172.3,156.2,138.0,137.3,135.3,134.0$, 129.5, 128.3 (2C), 127.4 (2C), 116.0, 98.3, 92.8, 79.8, 67.7, 59.5, 58.6, 52.1, 50.5, 47.4, 45.3, 32.2, 28.5 (major rotamer, 3C). HRMS (ESI) calcd for $\mathrm{C}_{26} \mathrm{H}_{35} \mathrm{~N}_{2} \mathrm{O}_{5}\left[\mathrm{M}+\mathrm{H}^{+}\right]$455.2540, found 455.2575 .

Tert-butyl

$\left(\left(\left(3 S^{*}, 3 a R^{*}, 6 R^{*}, 7 a R^{*}\right)-2-\right.\right.$ benzyl-3-((2methoxyphenoxy)methyl)-2,3,7,7a-tetrahydro-3a,6-

epoxyisoindol-6(1H)-yl)methyl)(methyl)carbamate (8). General Method A was applied to tert-butyl $\left(\left(\left(3 S^{\star}, 3 a R^{\star}, 6 R^{\star}, 7 a R^{\star}\right)-2-\right.\right.$ benzyl-3-(hydroxymethyl)-2,3,7,7a-tetrahydro-3a,6epoxyisoindol-6(1H)-yl)methyl)(methyl)carbamate (5) (1.00 g, $2.49 \mathrm{mmol})$ and 2-methoxyphenol $(335 \mu \mathrm{L}, 3 \mathrm{mmol})$. The reaction was concentrated and the crude was purified by flash column chromatography (EtOAc/heptane 4:1, $R_{f}=0.2$ ) to give the desired compound as a colorless oil $(762 \mathrm{mg}, 60 \%) .{ }^{1} \mathrm{H}$ NMR $(400 \mathrm{MHz}$, $\left.\mathrm{CDCl}_{3}\right) \delta 7.43-7.36(\mathrm{~m}, 2 \mathrm{H}), 7.36-7.29(\mathrm{~m}, 2 \mathrm{H}), 7.29-7.23(\mathrm{~m}$, $1 \mathrm{H}), 7.00-6.88(\mathrm{~m}, 4 \mathrm{H}), 6.61(\mathrm{dd}, J=10.0,5.7 \mathrm{~Hz}, 1 \mathrm{H}), 6.26$ (dd, $\mathrm{J}=18.8,5.8 \mathrm{~Hz}, 1 \mathrm{H}), 4.20-4.06(\mathrm{~m}, 3 \mathrm{H}), 3.83(\mathrm{~s}, 3 \mathrm{H}), 3.81-$ $3.70(\mathrm{~m}, 2 \mathrm{H}), 3.36(\mathrm{dd}, \mathrm{J}=6.6,4.9 \mathrm{~Hz}, 1 \mathrm{H}), 3.25(\mathrm{dd}, \mathrm{J}=7.8,6.2$ $\mathrm{Hz}, 1 \mathrm{H}), 2.99(\mathrm{~d}, \mathrm{~J}=7.7 \mathrm{~Hz}, 3 \mathrm{H}), 2.32-2.13(\mathrm{~m}, 2 \mathrm{H}), 1.58-1.28$ (m, $12 \mathrm{H}) .{ }^{13} \mathrm{C}$ NMR $\left(101 \mathrm{MHz}, \mathrm{CDCl}_{3}\right) \delta 156.3$ (major rotamer), 155.7 (minor rotamer), 150.2, 148.6, 139.6 (major rotamer), 139.4 (minor rotamer), 136.4 (major rotamer), 136.1 (minor rotamer), 136.0 (major rotamer), 135.7 (minor rotamer), 129.0 (minor rotamer), 128.9 (major rotamer), 128.3 (2C), 127.0 (2C), 121.8, 121.0, 115.1 (major rotamer), 115.0 (minor rotamer), 112.64 (major rotamer), 112.59 (minor rotamer), 98.4, 92.0 (major rotamer), 91.5 (minor rotamer), 79.7 (major rotamer), 79.4 (minor rotamer), 70.4 (major rotamer), 70.2 (minor rotamer), 63.7 (major rotamer), 63.6 (minor rotamer), 59.9 (major rotamer), 59.1 (minor rotamer), 56.1, 50.5, 49.7, 44.2, 35.9 (major rotamer), 35.8 (minor rotamer), 32.4, 28.53 (minor rotamer, $3 \mathrm{C}$ ), 28.48 (major rotamer, 3C). HRMS (ESI) calcd for $\mathrm{C}_{30} \mathrm{H}_{39} \mathrm{~N}_{2} \mathrm{O}_{5}\left[\mathrm{M}+\mathrm{H}^{+}\right] 507.2853$, found 507.2858 .

1-((3S*,3aR*,6S*,7aR*)-2-benzyl-3-((2-

Methoxyphenoxy)methyl)hexahydro-3a,6-epoxyisoindol-6(1H)$y l)-N$-methylmethanamine (S5). General Method $\mathbf{F}$ was applied to tert-butyl $\left(\left(\left(3 S^{\star}, 3 a R^{\star}, 6 R^{\star}, 7 a R^{\star}\right)-2\right.\right.$-benzyl-3-((2- 
methoxyphenoxy)methyl)-2,3,7,7a-tetrahydro-3a,6-

epoxyisoindol-6(1H)-yl)methyl)(methyl)carbamate (8) $(418 \mathrm{mg}$, $0.825 \mathrm{mmol}$ ). The crude mixture was purified by flash column chromatography $\left(\mathrm{CH}_{2} \mathrm{Cl}_{2} / \mathrm{MeOH}\right.$ with $5 \%$ of $\mathrm{NH}_{4} \mathrm{OH}(25 \%$ aq.) 95:5, $\left.R_{\mathrm{f}}=0.25\right)$ to give the desired compound as a white solid (241 mg, $72 \%) .{ }^{1} \mathrm{H} \mathrm{NMR}\left(400 \mathrm{MHz}, \mathrm{CDCl}_{3}\right) \delta 7.40-7.36(\mathrm{~m}, 2 \mathrm{H})$, $7.34-7.20(\mathrm{~m}, 3 \mathrm{H}), 7.02-6.86(\mathrm{~m}, 4 \mathrm{H}), 6.60(\mathrm{~d}, \mathrm{~J}=5.8 \mathrm{~Hz}, 1 \mathrm{H})$, $6.30(\mathrm{~d}, \mathrm{~J}=5.8 \mathrm{~Hz}, 1 \mathrm{H}), 4.16(\mathrm{~d}, \mathrm{~J}=13.0 \mathrm{~Hz}, 1 \mathrm{H}), 4.13-4.05(\mathrm{~m}$, $2 \mathrm{H}), 3.82(\mathrm{~s}, 3 \mathrm{H}), 3.73(\mathrm{~d}, \mathrm{~J}=12.9 \mathrm{~Hz}, 1 \mathrm{H}), 3.36(\mathrm{dd}, \mathrm{J}=6.5,4.8$ $\mathrm{Hz}, 1 \mathrm{H}), 3.24(\mathrm{dd}, \mathrm{J}=7.7,6.1 \mathrm{~Hz}, 1 \mathrm{H}), 3.15(\mathrm{~d}, \mathrm{~J}=12.6 \mathrm{~Hz}, 1 \mathrm{H})$, $3.06(\mathrm{~d}, \mathrm{~J}=12.5 \mathrm{~Hz}, 1 \mathrm{H}), 2.53(\mathrm{~s}, 3 \mathrm{H}), 2.34-2.18(\mathrm{~m}, 2 \mathrm{H}), 1.69$ - 1.63 (br. s, $1 \mathrm{H}), 1.59$ (dd, $J=11.3,2.6 \mathrm{~Hz}, 1 \mathrm{H}), 1.40$ (dd, $J=$ $11.3,7.1 \mathrm{~Hz}, 1 \mathrm{H}) .{ }^{13} \mathrm{C}$ NMR $\left(101 \mathrm{MHz}, \mathrm{CDCl}_{3}\right) \delta 150.2,148.6$, 139.7, 136.7, 135.5, 129.0, 128.3 (2C), 127.0 (2C), 121.7, 121.0, $114.9,112.6,98.4,90.7,70.3,63.7,60.0,59.0,56.1,54.4,44.4$, 37.2, 32.8. HRMS (ESI) calcd for $\mathrm{C}_{25} \mathrm{H}_{31} \mathrm{~N}_{2} \mathrm{O}_{3}\left[\mathrm{M}+\mathrm{H}^{+}\right]$407.2329, found 407.2328 .

$N-\left(\left(\left(3 S^{*}, 3 a R^{*}, 6 S^{*}, 7 a R^{*}\right)-2-(C y c l o h e x y l m e t h y l)-3-((2-\right.\right.$ methoxyphenoxy)methyl)hexahydro-3a,6-epoxyisoindol-6(1H)yl)methyl)- $N$-methylacetamide (9). General Method $\mathbf{H}$ was

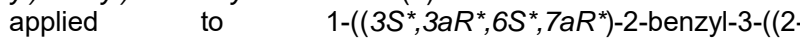
methoxyphenoxy)methyl)hexahydro-3a,6-epoxyisoindol-6(1H)yl)- $N$-methylmethanamine (S5) $(131 \mathrm{mg}, 0.32 \mathrm{mmol})$ and acetyl chloride $(28 \mu \mathrm{L}, 0.39 \mathrm{mmol})$ then the crude $(145 \mathrm{mg}, 0.32 \mathrm{mmol})$ was subjected to conditions in General Method B. General Method D was then applied to the crude ( $38 \mathrm{mg}, 0.09 \mathrm{mmol}$ ) and cyclohexanecarbaldehyde $(14 \mu \mathrm{L}, 0.11 \mathrm{mmol})$. The reaction mixture was purified directly by preparative HPLC to give the desired compound as a colorless oil $(28.2 \mathrm{mg}, 66 \%$ over three steps). ${ }^{1} \mathrm{H}$ NMR (400 MHz, $\left.\mathrm{CDCl}_{3}\right) \delta 6.94-6.85$ (m, 4H), $4.02-$ $3.84(\mathrm{~m}, 3 \mathrm{H}), 3.83(\mathrm{~s}, 3 \mathrm{H}), 3.62-3.49(\mathrm{~m}, 1 \mathrm{H}), 3.23-3.14(\mathrm{~m}$, $2 \mathrm{H}$ ), $3.13(\mathrm{~s}, 2 \mathrm{H}$, major rotamer), $3.03(\mathrm{~s}, 1 \mathrm{H}$, minor rotamer), 2.59 (td, $\mathrm{J}=11.8,9.3 \mathrm{~Hz}, 1 \mathrm{H}), 2.45-2.31(\mathrm{~m}, 2 \mathrm{H}), 2.12(\mathrm{~s}, 2 \mathrm{H}$, major rotamer), $2.11(\mathrm{~s}, 1 \mathrm{H}$, minor rotamer), $2.02-1.34(\mathrm{~m}, 13 \mathrm{H}), 1.30$ - $1.04(\mathrm{~m}, 3 \mathrm{H}), 0.95-0.67(\mathrm{~m}, 2 \mathrm{H}) .{ }^{13} \mathrm{C}$ NMR $\left(101 \mathrm{MHz}^{\left.-\mathrm{CDCl}_{3}\right)}\right.$ $\delta 171.4$ (minor rotamer), 171.2 (major rotamer), 149.8, 148.7 (major rotamer), 148.6 (minor rotamer), 121.34 (minor rotamer), 121.31 (major rotamer), 120.97 (major rotamer), 120.95 (minor rotamer), 113.83 (major rotamer), 113.77 (minor rotamer), 112.3, 96.0 (minor rotamer), 95.8 (major rotamer), 88.1 (major rotamer), 87.6 (minor rotamer), 70.0 (major rotamer), 69.8 (minor rotamer), 65.7 (major rotamer), 65.5 (minor rotamer), 63.3 (major rotamer), 63.1 (minor rotamer), 59.9 (major rotamer), 59.7 (minor rotamer), 56.1 (major rotamer), 53.6 (minor rotamer), 49.3, 47.01 (major rotamer), 46.96 (minor rotamer), 37.7 (major rotamer), 37.62 (major rotamer), 37.59 (minor rotamer), 36.98 (major rotamer), 36.95 (minor rotamer), 35.5 (minor rotamer), 32.9 (major rotamer), 32.7 (minor rotamer), 32.22 (major rotamer), 32.17 (minor rotamer), 31.60 (minor rotamer), 31.58 (major rotamer), 28.80 (minor rotamer), 28.78 (major rotamer), 26.95 (major rotamer), 26.94 (minor rotamer), 26.28 (major rotamer), 26.25 (minor rotamer), 26.1, 21.84 (minor rotamer), 21.82 (major rotamer). HRMS (ESI) calcd for $\mathrm{C}_{27} \mathrm{H}_{41} \mathrm{~N}_{2} \mathrm{O}_{4}\left[\mathrm{M}+\mathrm{H}^{+}\right]$457.3061, found 457.3071 .

$N-\left(\left(\left(3 S^{*}, 3 a R^{*}, 6 S^{*}, 7 a R^{*}\right)-3-((2-M e t h o x y p h e n o x y) m e t h y l)-2-\right.\right.$ (thiophene-2-carbonyl)hexahydro-3a,6-epoxyisoindol-6(1H)yl)methyl)- $N$-methylacetamide (10). General Method $\mathbf{H}$ was

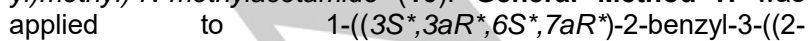
methoxyphenoxy)methyl)hexahydro-3a,6-epoxyisoindol-6(1H)$\mathrm{yl})-\mathrm{N}$-methylmethanamine (S5) $(131 \mathrm{mg}, 0.32 \mathrm{mmol}$ ) and acetyl chloride $(28 \mu \mathrm{L}, 0.39 \mathrm{mmol})$ then the crude $(145 \mathrm{mg}, 0.32 \mathrm{mmol})$ was subjected to conditions in General Method B. General Method $\mathbf{H}$ was then applied to the crude $(28.3 \mathrm{mg}, 0.08 \mathrm{mmol})$ and thiophene-2-carbonyl chloride $(10 \mu \mathrm{L}, 0.09 \mathrm{mmol})$. The reaction mixture was purified by preparative HPLC to give the desired compound as an amorphous white solid (24 mg, $65 \%$ over three steps). ${ }^{1} \mathrm{H}$ NMR $\left(400 \mathrm{MHz}, \mathrm{CDCl}_{3}\right) \delta 7.61-7.51(\mathrm{~m}$, $1 \mathrm{H}), 7.48(\mathrm{~d}, \mathrm{~J}=5.0 \mathrm{~Hz}, 1 \mathrm{H}), 7.12-7.01(\mathrm{~m}, 1 \mathrm{H}), 6.96-6.78(\mathrm{~m}$, $4 \mathrm{H}), 4.88(\mathrm{~s}, 1 \mathrm{H}), 4.64(\mathrm{td}, \mathrm{J}=10.2,9.7,3.2 \mathrm{~Hz}, 1 \mathrm{H}), 4.31(\mathrm{t}, \mathrm{J}=$ $9.5 \mathrm{~Hz}, 1 \mathrm{H}), 4.09(\mathrm{dd}, \mathrm{J}=10.0,2.0 \mathrm{~Hz}, 1 \mathrm{H}), 3.86-3.63(\mathrm{~m}, 5 \mathrm{H})$, $3.37(\mathrm{q}, \mathrm{J}=8.9 \mathrm{~Hz}, 1 \mathrm{H}), 3.27-3.11(\mathrm{~m}, 1 \mathrm{H}), 3.07(\mathrm{~s}, 2.3 \mathrm{H}$, major rotamer), $2.98(\mathrm{~s}, 0.7 \mathrm{H}$, minor rotamer), $2.11(\mathrm{~s}, 2.3 \mathrm{H}$, major rotamer), $2.10(\mathrm{~s}, 0.7 \mathrm{H}$, minor rotamer), $2.06-1.86(\mathrm{~m}, 3 \mathrm{H}), 1.74$ - $1.46(\mathrm{~m}, 3 \mathrm{H}) .{ }^{13} \mathrm{C}$ NMR $\left(101 \mathrm{MHz}, \mathrm{CDCl}_{3}\right) \delta 171.30$ (major rotamer), 171.28 (minor rotamer), 161.9, 149.6, 148.3, 139.5, $130.3,130.2$, 127.4, 121.5, 121.1, 113.5, 112.1, 93.4 (minor rotamer), 93.3 (major rotamer), 88.3 (major rotamer), 87.9 (minor rotamer), 66.9, 61.3 (major rotamer), 61.1 (minor rotamer), 56.9 (major rotamer), 56.7 (minor rotamer), 56.0 (major rotamer), 53.4 (minor rotamer), 49.3, 46.9 (minor rotamer), 46.8 (major rotamer), 42.1 (major rotamer), 41.6 (minor rotamer), 37.9 (major rotamer), 35.5 (minor rotamer), 32.3 (minor rotamer), 31.9 (major rotamer), 28.6, 21.9 (minor rotamer) 21.8 (major rotamer). HRMS (ESI) calcd for $\mathrm{C}_{25} \mathrm{H}_{31} \mathrm{~N}_{2} \mathrm{O}_{5} \mathrm{~S}\left[\mathrm{M}+\mathrm{H}^{+}\right]$471.1948, found 471.1948.

$N-\left(\left(\left(3 S^{*}, 3 a R^{*}, 6 S^{*}, 7 a R^{*}\right)-3-((2-M e t h o x y p h e n o x y) m e t h y l)-2-\right.\right.$ (methylsulfonyl)hexahydro-3a,6-epoxyisoindol-6(1H)-yl)methyl)$\mathrm{N}$-methylacetamide (11). General Method $\mathbf{H}$ was applied to 1$\left(\left(3 S^{*}, 3 a R^{*}, 6 S^{*}, 7 a R^{\star}\right)-2-\right.$ benzyl-3- $((2-$

methoxyphenoxy)methyl)hexahydro-3a,6-epoxyisoindol-6(1H)$\mathrm{yl})-\mathrm{N}$-methylmethanamine (S5) $(131 \mathrm{mg}, 0.32 \mathrm{mmol})$ and acetyl chloride $(28 \mu \mathrm{L}, 0.39 \mathrm{mmol})$ then the crude $(145 \mathrm{mg}, 0.32 \mathrm{mmol})$ was subjected to conditions in General Method B. General Method $\mathbf{K}$ was then applied to the crude $(32.3 \mathrm{mg}, 0.09 \mathrm{mmol})$ and methanesulfonyl chloride $(8 \mu \mathrm{L}, 0.11 \mathrm{~mol})$. The reaction mixture was purified by preparative HPLC to give the desired compound as an amorphous white solid $(24 \mathrm{mg}, 61 \%$ over three steps). ${ }^{1} \mathrm{H}$ NMR (400 MHz, $\left.\mathrm{CDCl}_{3}\right) \delta 6.97-6.83$ (m, 4H), $4.26-$ $4.08(\mathrm{~m}, 3 \mathrm{H}), 3.89-3.63(\mathrm{~m}, 6 \mathrm{H}), 3.09(\mathrm{~s}, 2.2 \mathrm{H}$, major rotamer $)$, $3.00(\mathrm{~s}, 0.8 \mathrm{H}$, minor rotamer), $2.99-2.88(\mathrm{~m}, 1 \mathrm{H}), 2.84(\mathrm{~d}, \mathrm{~J}=$ $8.4 \mathrm{~Hz}, 4 \mathrm{H}), 2.12(\mathrm{~s}, 2.2 \mathrm{H}$, major rotamer), $2.10(\mathrm{~s}, 0.8 \mathrm{H}$, minor rotamer), 1.93 (tdd, $J=15.3,10.2,6.5 \mathrm{~Hz}, 3 \mathrm{H}), 1.73-1.45(\mathrm{~m}$, $3 \mathrm{H}) \cdot{ }^{13} \mathrm{C}$ NMR $\left(101 \mathrm{MHz}, \mathrm{CDCl}_{3}\right) \delta 171.2,149.64$ (major rotamer), 149.60 (minor rotamer), 148.05 (major rotamer), 148.03 (minor rotamer), 121.7, 121.11 (minor rotamer), 121.05 (major rotamer), $113.5,112.22$ (minor rotamer), 112.19 (major rotamer), 95.3 (minor rotamer), 95.1 (major rotamer), 88.7 (major rotamer), 88.2 (minor rotamer), 69.7 (minor rotamer), 69.5 (major rotamer), 62.0 (major rotamer), 61.9 (minor rotamer), 56.07 (major rotamer), 56.05 (minor rotamer), 55.98 (major rotamer), 55.8 (minor rotamer), 53.4 (minor rotamer), 49.4 (major rotamer), 46.40 (minor rotamer), 46.35 (major rotamer ), 40.9 (major rotamer), 40.7 (minor rotamer), 37.8 (major rotamer), 35.8 (minor rotamer), 34.5 (major rotamer), 33.9 (minor rotamer), 32.5 (minor rotamer), 32.4 (major rotamer), 28.2, 21.8. HRMS (ESI) calcd for $\mathrm{C}_{21} \mathrm{H}_{31} \mathrm{~N}_{2} \mathrm{O}_{6} \mathrm{~S}\left[\mathrm{M}+\mathrm{H}^{+}\right]$439.1897, found 439.1899.

$\left(3 S^{*}, 3 a R^{*}, 6 S^{*}, 7 a R^{*}\right)-N-C y c l o p e n t y l-3-((2-$

methoxyphenoxy)methyl)-6-((N-

methylacetamido)methyl)hexahydro-3a,6-epoxyisoindole-2(3H)carboxamide (12). General Method $\mathbf{H}$ was applied to 1$\left(\left(3 S^{*}, 3 a R^{*}, 6 S^{*}, 7 a R^{\star}\right)-2\right.$-benzyl-3-((2-

methoxyphenoxy)methyl)hexahydro-3a,6-epoxyisoindol-6(1H)$\mathrm{yl})-N$-methylmethanamine (S5) $(131 \mathrm{mg}, 0.32 \mathrm{mmol})$ and acetyl chloride $(28 \mu \mathrm{L}, 0.39 \mathrm{mmol})$ then the crude $(145 \mathrm{mg}, 0.32 \mathrm{mmol})$ was subjected to conditions in General Method B. General Method G was then applied to the crude $(40.6 \mathrm{mg}, 0.11 \mathrm{mmol})$ and cyclopentyl isocyanate $(15 \mu \mathrm{L}, 0.135 \mathrm{mmol})$. The reaction mixture was purified by preparative HPLC to give the desired compound as an amorphous white solid (32 mg, $60 \%$ over three 
steps). ${ }^{1} \mathrm{H}$ NMR $\left(400 \mathrm{MHz}, \mathrm{CDCl}_{3}\right) \delta 6.96-6.79(\mathrm{~m}, 4 \mathrm{H}), 4.78-$ $4.19(\mathrm{~m}, 3 \mathrm{H}), 4.12-4.01(\mathrm{~m}, 1 \mathrm{H}), 3.98$ (ddd, $J=9.7,5.3,3.6 \mathrm{~Hz}$, $1 \mathrm{H}), 3.81(\mathrm{~s}, 3 \mathrm{H}), 3.79-3.55(\mathrm{~m}, 3 \mathrm{H}), 3.07(\mathrm{~s}, 2.2 \mathrm{H}$, major rotamer), $2.98(\mathrm{~s}, 0.8 \mathrm{H}$, minor rotamer), $2.96-2.68(\mathrm{~m}, 2 \mathrm{H}), 2.10$ $(\mathrm{s}, 2.2 \mathrm{H}$, major rotamer $), 2.08(\mathrm{~s}, 0.8 \mathrm{H}$, minor rotamer $), 2.02-$ $1.78(\mathrm{~m}, 5 \mathrm{H}), 1.69-1.51(\mathrm{~m}, 6 \mathrm{H}), 1.46(\mathrm{dt}, J=12.0,3.1 \mathrm{~Hz}, 1 \mathrm{H})$, $1.40-1.25(\mathrm{~m}, 2 \mathrm{H}) .{ }^{13} \mathrm{C}$ NMR $\left(101 \mathrm{MHz}, \mathrm{CDCl}_{3}\right) \delta 171.24$ (minor rotamer), 171.17 (major rotamer), 157.1 (major rotamer), 156.8 (minor rotamer), 149.5 (major rotamer), 149.4 (minor rotamer), 148.23 (minor rotamer), 148.19 (major rotamer), 121.5 (major rotamer), 121.4 (minor rotamer), 121.1 (minor rotamer), 121.1 (major rotamer), 113.6 (major rotamer), 113.4 (minor rotamer), 112.0, 94.3 (minor rotamer), 94.1 (major rotamer), 88.3 (major rotamer), 87.8 (minor rotamer), 60.0 (major rotamer), 59.8 (minor rotamer), 56.0 (minor rotamer), 55.9 (major rotamer), 53.7 (major rotamer), 53.4 (minor rotamer), 53.3, 52.4 (major rotamer), 52.3 (minor rotamer), 49.2, 46.0 (minor rotamer), 45.7 (major rotamer), 41.0 (major rotamer), 40.7 (minor rotamer), 37.8, 35.5, 33.7 (minor rotamer), 33.6 (major rotamer), 32.3 (minor rotamer), 32.0 (major rotamer), 28.7 (major rotamer), 28.6 (minor rotamer), 23.8 (major rotamer), 23.71 (minor rotamer), 23.69, 21.83 (minor rotamer), 21.79 (major rotamer). HRMS (ESI) calcd for $\mathrm{C}_{26} \mathrm{H}_{38} \mathrm{~N}_{3} \mathrm{O}_{5}\left[\mathrm{M}+\mathrm{H}^{+}\right]$472.2806, found 472.2809.

$N-\left(\left(\left(3 S^{*}, 3 a R^{*}, 6 S^{*}, 7 a R^{*}\right)-3-((2-M e t h o x y p h e n o x y) m e t h y l)-2-\right.\right.$ (pyridin-3-ylmethyl)hexahydro-3a,6-epoxyisoindol-6(1H)yl)methyl)- $N$-methylmethanesulfonamide (13). General Method $\mathbf{K}$ was applied to $1-\left(\left(3 S^{*}, 3 a R^{\star}, 6 S^{*}, 7 a R^{*}\right)-2\right.$-benzyl-3-((2methoxyphenoxy)methyl)hexahydro-3a,6-epoxyisoindol-6(1H)$\mathrm{yl})-\mathrm{N}$-methylmethanamine (S5) (105 mg, $0.258 \mathrm{mmol})$ and methanesulfonyl chloride $(24 \mu \mathrm{L}, 0.310 \mathrm{mmol})$ then the crude (125 $\mathrm{mg}, 0.25832 \mathrm{mmol}$ ) was subjected to conditions in General Method B. General Method D was then applied to the crude (26 $\mathrm{mg}, 0.066 \mathrm{mmol})$ and nicotinaldehyde $(6 \mu \mathrm{L}, 0.066 \mathrm{mmol})$. The reaction mixture was purified by preparative HPLC to give the desired compound as a colorless oil solid $(21 \mathrm{mg}, 65 \%$ over three steps). ${ }^{1} \mathrm{H}$ NMR $\left(400 \mathrm{MHz}, \mathrm{CDCl}_{3}\right) \delta 8.54(\mathrm{~d}, \mathrm{~J}=2.0 \mathrm{~Hz}, 1 \mathrm{H}), 8.47$ (dd, $J=4.8,1.6 \mathrm{~Hz}, 1 \mathrm{H}), 7.65$ (dt, $J=7.8,2.0 \mathrm{~Hz}, 1 \mathrm{H}$ ), 7.21 (dd, $\mathrm{J}=7.8,4.8 \mathrm{~Hz}, 1 \mathrm{H}), 6.98-6.84(\mathrm{~m}, 4 \mathrm{H}), 4.22(\mathrm{~d}, \mathrm{~J}=13.4 \mathrm{~Hz}$, $1 \mathrm{H}), 4.04(\mathrm{dd}, J=9.7,6.1 \mathrm{~Hz}, 1 \mathrm{H}), 3.96(\mathrm{dd}, J=9.6,5.5 \mathrm{~Hz}, 1 \mathrm{H})$, $3.80(\mathrm{~s}, 3 \mathrm{H}), 3.66(\mathrm{~d}, \mathrm{~J}=13.4 \mathrm{~Hz}, 1 \mathrm{H}), 3.51(\mathrm{~d}, \mathrm{~J}=14.6 \mathrm{~Hz}, 1 \mathrm{H})$, $3.42(\mathrm{t}, \mathrm{J}=5.8 \mathrm{~Hz}, 1 \mathrm{H}), 3.36(\mathrm{~d}, \mathrm{~J}=14.6 \mathrm{~Hz}, 1 \mathrm{H}), 3.04-2.95(\mathrm{~m}$, $4 \mathrm{H}), 2.81(\mathrm{~s}, 3 \mathrm{H}), 2.40(\mathrm{dtd}, \mathrm{J}=11.0,7.2,4.0 \mathrm{~Hz}, 1 \mathrm{H}), 2.17-1.96$ $(\mathrm{m}, 2 \mathrm{H}), 1.84-1.68(\mathrm{~m}, 2 \mathrm{H}), 1.71-1.62(\mathrm{~m}, 2 \mathrm{H}), 1.65-1.55(\mathrm{~m}$, $1 \mathrm{H}) ;{ }^{13} \mathrm{C}$ NMR $\left(101 \mathrm{MHz}, \mathrm{CDCl}_{3}\right) \delta 150.3,149.9,148.5,148.4$, 136.8, 134.8, 123.4, 121.6, 121.0, 113.9, 112.2, 96.1, 87.6, 70.5, 64.3, 59.1, 56.9, 56.0, 52.4, 46.8, 37.6, 36.8, 35.4, 32.6, 29.0. HRMS (ESI) calcd for $\mathrm{C}_{25} \mathrm{H}_{34} \mathrm{~N}_{3} \mathrm{O}_{5} \mathrm{~S}\left[\mathrm{M}+\mathrm{H}^{+}\right] 488.2214$, found 488.2217 .

$\left(3 S^{\star}, 3 a R^{*}, 6 S^{\star}, 7 a R^{*}\right)-N-H e x y l-3-((2-m e t h o x y p h e n o x y) m e t h y l)-6-$ ((N-methylmethylsulfonamido)methyl)hexahydro-3a,6-

epoxyisoindole-2(3H)-carboxamide (14). General Method $\mathbf{K}$ was

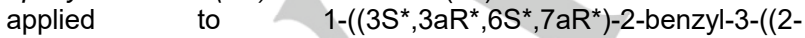
methoxyphenoxy)methyl)hexahydro-3a,6-epoxyisoindol-6 $(1 H)$ $\mathrm{yl})-\mathrm{N}$-methylmethanamine (S5) (105 mg, $0.258 \mathrm{mmol})$ and methanesulfonyl chloride $(24 \mu \mathrm{L}, 0.310 \mathrm{mmol})$ then the crude (125 $\mathrm{mg}, 0.25832 \mathrm{mmol}$ ) was subjected to conditions in General Method B. General Method $\mathbf{G}$ was then applied to the crude (32 $\mathrm{mg}, 0.081 \mathrm{mmol})$ and hexyl isocyanate $(14 \mu \mathrm{L}, 0.098 \mathrm{mmol})$. The reaction mixture was purified by preparative HPLC to give the desired compound as a colorless oil solid $(15 \mathrm{mg}, 35 \%$ over three steps). ${ }^{1} \mathrm{H}$ NMR (400 MHz, $\left.\mathrm{CDCl}_{3}\right) \delta 6.96-6.83(\mathrm{~m}, 4 \mathrm{H}), 4.76$ (br. $\mathrm{s}, 1 \mathrm{H}), 4.46(\mathrm{t}, \mathrm{J}=3.9 \mathrm{~Hz}, 1 \mathrm{H}), 4.36(\mathrm{dd}, \mathrm{J}=9.9,3.5 \mathrm{~Hz}, 1 \mathrm{H}), 3.99$ (dd, $J=9.8,4.2 \mathrm{~Hz}, 1 \mathrm{H}), 3.82(\mathrm{~s}, 3 \mathrm{H}), 3.65(\mathrm{t}, J=8.9 \mathrm{~Hz}, 1 \mathrm{H})$, $3.48(\mathrm{~d}, \mathrm{~J}=14.6 \mathrm{~Hz}, 1 \mathrm{H}), 3.41(\mathrm{~d}, \mathrm{~J}=14.6 \mathrm{~Hz}, 1 \mathrm{H}), 3.30-3.10$ $(\mathrm{m}, 2 \mathrm{H}), 3.03-2.96(\mathrm{~m}, 1 \mathrm{H}), 2.98(\mathrm{~s}, 3 \mathrm{H}), 2.90-2.79(\mathrm{~m}, 4 \mathrm{H})$, $1.98-1.82(\mathrm{~m}, 3 \mathrm{H}), 1.85-1.71(\mathrm{~m}, 2 \mathrm{H}), 1.70-1.58(\mathrm{~m}, 1 \mathrm{H})$, $1.55-1.40(\mathrm{~m}, 2 \mathrm{H}), 1.36-1.17(\mathrm{~m}, 6 \mathrm{H}), 0.90-0.81(\mathrm{~m}, 3 \mathrm{H}) ;{ }^{13} \mathrm{C}$ NMR $\left(101 \mathrm{MHz}, \mathrm{CDCl}_{3}\right) \delta 157.5,149.4,148.2,121.5,121.1$, $113.1,111.9,94.5,87.7,68.8,60.0,56.0,53.6,52.3,45.8,40.9$, $40.7,36.9,35.3,32.3,31.7,30.4,28.7,26.8,22.7,14.2$. HRMS (ESI) calcd for $\mathrm{C}_{26} \mathrm{H}_{42} \mathrm{~N}_{3} \mathrm{O}_{5} \mathrm{~S}\left[\mathrm{M}+\mathrm{H}^{+}\right]$524.2789, found 524.2792.

Tert-butyl

$\left(\left(\left(3 S^{*}, 3 a R^{*}, 6 S^{*}, 7 a R^{*}\right)-2-(2-f l u o r o b e n z y l)-3-\right.\right.$ (hydroxymethyl)hexahydro-3a,6-epoxyisoindol-6(1H)-

yl)methyl)(methyl)carbamate (15). General Method B was

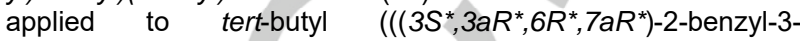
(hydroxymethyl)-2,3,7,7a-tetrahydro-3a,6-epoxyisoindol-6(1H)yl)methyl)(methyl)carbamate (5) (1.03g, $2.56 \mathrm{mmol})$ and then the crude $(165 \mathrm{mg}, 0.53 \mathrm{mmol})$ and 2-fluorobenzaldehyde $(83 \mu \mathrm{L}$, $0.79 \mathrm{mmol}$ ) were subjected to General Method D. The benzylated compound was purified by flash column chromatography $\left(\mathrm{CH}_{2} \mathrm{Cl}_{2} / \mathrm{MeOH}\right.$ with $5 \%$ of $\mathrm{NH}_{4} \mathrm{OH}(25 \%$ aq $)$ 98:2, $\left.R_{\mathrm{f}}=0.25\right)$ to give the title compound as a colorless oil (196 $\mathrm{mg}, 88 \%) .{ }^{1} \mathrm{H}$ NMR $\left(400 \mathrm{MHz}, \mathrm{CDCl}_{3}\right) \delta 7.33-7.21(\mathrm{~m}, 2 \mathrm{H}), 7.10$ $(\mathrm{t}, \mathrm{J}=7.4 \mathrm{~Hz}, 1 \mathrm{H}), 7.07-7.00(\mathrm{~m}, 1 \mathrm{H}), 3.98(\mathrm{~d}, \mathrm{~J}=13.0 \mathrm{~Hz}, 1 \mathrm{H})$, $3.67(\mathrm{td}, \mathrm{J}=11.7,9.9,6.0 \mathrm{~Hz}, 3 \mathrm{H}), 3.59-3.54(\mathrm{~m}, 1 \mathrm{H}), 3.44(\mathrm{dd}$, $\mathrm{J}=24.0,15.1 \mathrm{~Hz}, 1 \mathrm{H}), 3.04(\mathrm{dd}, \mathrm{J}=13.7,5.4 \mathrm{~Hz}, 2 \mathrm{H}), 2.95$ (s, $3 \mathrm{H}), 2.28-2.13(\mathrm{~m}, 2 \mathrm{H}), 1.89(\mathrm{dt}, J=12.8,6.3 \mathrm{~Hz}, 1 \mathrm{H}), 1.80(\mathrm{dt}$, $\mathrm{J}=11.9,8.9 \mathrm{~Hz}, 1 \mathrm{H}), 1.71-1.55(\mathrm{~m}, 3 \mathrm{H}), 1.46-1.37(\mathrm{~m}, 10 \mathrm{H})$. ${ }^{13} \mathrm{C}$ NMR $\left(101 \mathrm{MHz}, \mathrm{CDCl}_{3}\right) \delta 161.4(\mathrm{~d}, \mathrm{~J}=246.6 \mathrm{~Hz}), 156.3$ (minor rotamer), 155.8 (major rotamer), $131.3(\mathrm{~d}, \mathrm{~J}=6.9 \mathrm{~Hz}$ ), $129.2(\mathrm{~d}, \mathrm{~J}=8.2 \mathrm{~Hz}$ ), $125.6(\mathrm{~d}, \mathrm{~J}=14.4 \mathrm{~Hz}), 124.0(\mathrm{~d}, \mathrm{~J}=3.6 \mathrm{~Hz})$, $115.6(\mathrm{~d}, \mathrm{~J}=22.0 \mathrm{~Hz}$ ), 95.9, 88.6 (minor rotamer), 88.4 (major rotamer), 79.6 (major rotamer), 79.4 (minor rotamer), 66.4 (minor rotamer), 66.3 (major rotamer), 59.5, 58.8 (minor rotamer), 58.7 (major rotamer), 51.6 (major rotamer), $51.5(\mathrm{~d}, \mathrm{~J}=1.7 \mathrm{~Hz}), 50.7$ (minor rotamer), 47.3, 37.5, 35.8 (minor rotamer), 35.7 (major rotamer), 32.7 (major rotamer), 32.4 (minor rotamer), 29.9, 28.5 (3C). HRMS (ESI) calcd for $\mathrm{C}_{23} \mathrm{H}_{34} \mathrm{FN}_{2} \mathrm{O}_{4}\left[\mathrm{M}+\mathrm{H}^{+}\right] 421.2497$, found 421.2498 .

Tert-butyl $\quad\left(\left(\left(3 S^{*}, 3 a R^{*}, 6 S^{*}, 7 a R^{*}\right)-2-(2-\right.\right.$ fluorobenzyl)-3-(((6methylpyridin-3-yl)oxy)methyl)hexahydro-3a,6-epoxyisoindol-

6(1H)-yl)methyl)(methyl)carbamate (S6). General Method A was applied to tert-butyl $\left(\left(\left(3 S^{*}, 3 a R^{\star}, 6 S^{*}, 7 a R^{\star}\right)-2-(2-\right.\right.$ fluorobenzyl)-3(hydroxymethyl)hexahydro-3a,6-epoxyisoindol-6(1H)-

yl)methyl)(methyl)carbamate (9) $(80 \mathrm{mg}, 0.19 \mathrm{mmol})$ and 5 hydroxy-2-methylpyridine $(25 \mathrm{mg}, 0.23 \mathrm{mmol})$. The crude was purified by flash column chromatography $\left(\mathrm{CH}_{2} \mathrm{Cl}_{2} / \mathrm{MeOH}\right.$ with $5 \%$ of $\mathrm{NH}_{4} \mathrm{OH}\left(25 \%\right.$ aq.) $\left.98: 2, R_{\mathrm{f}}=0.2\right)$ to give an impure compound which was used in the next step without further purification.

$N-\left(\left(\left(3 S^{*}, 3 a R^{*}, 6 S^{*}, 7 a R^{*}\right)-2-(2-\right.\right.$ Fluorobenzyl)-3-(((6methylpyridin-3-yl)oxy)methyl)hexahydro-3a,6-epoxyisoindol$6(1 H)$-yl)methyl)-N-methylpentan-1-amine (16). General Method F was applied to $\left(\left(\left(3 S^{*}, 3 a R^{\star}, 6 S^{\star}, 7 a R^{\star}\right)-2-(2-f l u o r o b e n z y l)-3-((6\right.\right.$ methylpyridin-3-yl)oxy)methyl)hexahydro-3a,6-epoxyisoindol$6(1 H)$-yl)methyl)(methyl)carbamate (S6) (95 mg, $0.19 \mathrm{mmol})$. The crude $(20 \mathrm{mg}, 0.05 \mathrm{mmol}$ ) was then directly subjected to General Method D and valeraldehyde $(6 \mu \mathrm{L}, 0.058 \mathrm{mmol})$ and purified directly by preparative HPLC to give the desired compound as a colorless oil $\left(21 \mathrm{mg}, 90 \%\right.$ over three steps). ${ }^{1} \mathrm{H}$ NMR $(400 \mathrm{MHz}$, $\left.\mathrm{CDCl}_{3}\right) \delta 8,18(\mathrm{~d}, \mathrm{~J}=2.9 \mathrm{~Hz}, 1 \mathrm{H}), 7.33(\mathrm{td}, \mathrm{J}=7.5,1.8 \mathrm{~Hz}, 1 \mathrm{H})$, 7.21 (tdd, $J=7.5,5.2,1.8 \mathrm{~Hz}, 1 \mathrm{H}), 7.09$ (dd, $J=8.5,2.9 \mathrm{~Hz}, 1 \mathrm{H}$ ), $7.07-6.97(\mathrm{~m}, 3 \mathrm{H}), 4.06(\mathrm{dd}, \mathrm{J}=13.4,1.4 \mathrm{~Hz}, 1 \mathrm{H}), 4.01-3.89$ $(\mathrm{m}, 2 \mathrm{H}), 3.79(\mathrm{dd}, \mathrm{J}=13.3,1.2 \mathrm{~Hz}, 1 \mathrm{H}), 3.35(\mathrm{dd}, \mathrm{J}=6.1,4.5 \mathrm{~Hz}$, 1H), $3.06(\mathrm{dd}, J=8.3,6.9 \mathrm{~Hz}, 1 \mathrm{H}), 2.69(\mathrm{~d}, \mathrm{~J}=13.9 \mathrm{~Hz}, 1 \mathrm{H}), 2.62$ $(\mathrm{d}, \mathrm{J}=13.9 \mathrm{~Hz}, 1 \mathrm{H}), 2.52-2.43(\mathrm{~m}, 4 \mathrm{H}), 2.41-2.31(\mathrm{~m}, 2 \mathrm{H})$, $2.29(\mathrm{~s}, 3 \mathrm{H}), 2.27-2.19(\mathrm{~m}, 1 \mathrm{H}), 1.92(\mathrm{td}, \mathrm{J}=12.3,11.6,4.8 \mathrm{~Hz}$, $1 \mathrm{H}), 1.76-1.53(\mathrm{~m}, 5 \mathrm{H}), 1.52-1.39(\mathrm{~m}, 2 \mathrm{H}), 1.39-1.19(\mathrm{~m}, 4 \mathrm{H})$, 
$0.90(\mathrm{t}, \mathrm{J}=7.0 \mathrm{~Hz}, 3 \mathrm{H}) \cdot{ }^{13} \mathrm{C}$ NMR $\left(101 \mathrm{MHz}, \mathrm{CDCl}_{3}\right) \delta 161.3(\mathrm{~d}, \mathrm{~J}$ $=246.2 \mathrm{~Hz}), 153.2,150.6,136.9,131.5(\mathrm{~d}, \mathrm{~J}=4.6 \mathrm{~Hz}), 128.9$ (d, $\mathrm{J}=8.2 \mathrm{~Hz}), 125.8(\mathrm{~d}, \mathrm{~J}=14.7 \mathrm{~Hz}), 124.0(\mathrm{~d}, \mathrm{~J}=3.6 \mathrm{~Hz}), 123.4$, 122.2, $115.5(\mathrm{~d}, \mathrm{~J}=22.1 \mathrm{~Hz}), 95.5,88.4,69.1,64.7,60.5,59.2$, $59.1,52.1$ (d, $\mathrm{J}=2.2 \mathrm{~Hz}$ ), 47.1, 44.0, 38.1, 33.9, 29.8, 29.3, 27.1, 23.5, 22.8, 14.3. HRMS (ESI) calcd for $\mathrm{C}_{29} \mathrm{H}_{41} \mathrm{FN}_{3} \mathrm{O}_{2}\left[\mathrm{M}+\mathrm{H}^{+}\right]$ 482.3177 , found 482.3176 .

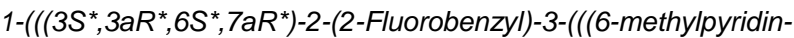
3-yl)oxy)methyl)hexahydro-3a,6-epoxyisoindol-6(1H)-yl)methyl)3-isopropyl-1-methylurea (17). General Method $\mathbf{F}$ was applied to $\left(\left(\left(3 S^{*}, 3 a R^{*}, 6 S^{*}, 7 a R^{*}\right)-2-(2-f l u o r o b e n z y l)-3-((6-m e t h y l p y r i d i n-3-\right.\right.$ yl)oxy)methyl)hexahydro-3a,6-epoxyisoindol-6(1H)yl)methyl)(methyl)carbamate (S6) $(95 \mathrm{mg}, 0.19 \mathrm{mmol})$. The crude (20 mg, $0.05 \mathrm{mmol}$ ) and isopropyl isocyanate $(5.7 \mu \mathrm{L}, 0.06 \mathrm{mmol})$ was then subjected to General Method $\mathbf{G}$ to give the desired compound as an amorphous white solid $(79 \mathrm{mg}, 86 \%$ over three steps). ${ }^{1} \mathrm{H}$ NMR $\left(400 \mathrm{MHz}, \mathrm{CDCl}_{3}\right) \delta 8.18(\mathrm{~d}, \mathrm{~J}=2.8 \mathrm{~Hz}, 1 \mathrm{H}), 7.30$ (td, $J=7.5,1.8 \mathrm{~Hz}, 1 \mathrm{H}), 7.22$ (tdd, $J=7.3,5.2,1.8 \mathrm{~Hz}, 1 \mathrm{H}$ ), 7.12 $-6.98(\mathrm{~m}, 4 \mathrm{H}), 5.43$ (broad s, $1 \mathrm{H}), 4.04(\mathrm{dd}, \mathrm{J}=13.3,1.4 \mathrm{~Hz}, 1 \mathrm{H})$, $3.97-3.86(\mathrm{~m}, 3 \mathrm{H}), 3.73(\mathrm{~d}, \mathrm{~J}=13.3 \mathrm{~Hz}, 1 \mathrm{H}), 3.49(\mathrm{~d}, \mathrm{~J}=16.1$ $\mathrm{Hz}, 1 \mathrm{H}), 3.44(\mathrm{~d}, \mathrm{~J}=17.0 \mathrm{~Hz}, 1 \mathrm{H}), 3.28(\mathrm{t}, \mathrm{J}=5.3 \mathrm{~Hz}, 1 \mathrm{H}), 3.06$ (d, J = 7.6 Hz, 1H), $2.92(\mathrm{~s}, 3 \mathrm{H}), 2.48(\mathrm{~s}, 3 \mathrm{H}), 2.44-2.35(\mathrm{~m}, 1 \mathrm{H})$, $2.23-2.12(\mathrm{~m}, 1 \mathrm{H}), 2.02-1.82(\mathrm{~m}, 1 \mathrm{H}), 1.74(\mathrm{ddd}, \mathrm{J}=12.0,8.8$, $4.1 \mathrm{~Hz}, 1 \mathrm{H}), 1.69-1.46(\mathrm{~m}, 4 \mathrm{H}), 1.15(\mathrm{~d}, \mathrm{~J}=6.2 \mathrm{~Hz}, 3 \mathrm{H}), 1.13$ (d, $\mathrm{J}=6.1 \mathrm{~Hz}, 3 \mathrm{H}) \cdot{ }^{13} \mathrm{C}$ NMR $\left(101 \mathrm{MHz}, \mathrm{CDCl}_{3}\right) \delta 161.3(\mathrm{~d}, \mathrm{~J}=246.2$ $\mathrm{Hz}), 159.2,153.1,150.6,136.5,131.6$ (d, J = 4.4 Hz), 129.2 (d, J $=8.1 \mathrm{~Hz}), 125.3(\mathrm{~d}, \mathrm{~J}=14.8 \mathrm{~Hz}), 124.1(\mathrm{~d}, \mathrm{~J}=3.6 \mathrm{~Hz}), 123.6$, 122.5, 115.6 (d, J = 22.1 Hz), 96.5, 89.0, 68.7, 64.7, 59.0, 52.2, 52.1 (d, $J=1.9 \mathrm{~Hz}), 47.0,42.7,37.0,36.8,32.1,29.3,23.8,23.7$, 23.3. HRMS (ESI) calcd for $\mathrm{C}_{28} \mathrm{H}_{38} \mathrm{FN}_{4} \mathrm{O}_{3}\left[\mathrm{M}+\mathrm{H}^{+}\right] 497.2922$, found 497.2922.

Tert-butyl $\quad\left(\left(\left(2 R^{\star}, 3 a R^{\star}, 5 a S^{*}, 6 S^{*}, 9 a R^{\star}\right)-5\right.\right.$-benzyl-6-hydroxy-2vinyl-2,3,3a,4,5,5a,6,7-octahydrofuro[3,2-c]indol-2-

yl)methyl)(methyl)carbamate (18). A stirred solution of tert-butyl $\left(\left(\left(3 S^{\star}, 3 a R^{\star}, 6 R^{\star}, 7 a R^{\star}\right)-2\right.\right.$-benzyl-3-((S)-1-hydroxybut-3-en-1-yl)2,3,7,7a-tetrahydro-3a,6-epoxyisoindol-6(1H)-

yl)methyl)(methyl)carbamate (6) $(180 \mathrm{mg}, 0.41 \mathrm{mmol})$ in $\mathrm{CH}_{2} \mathrm{Cl}_{2}$ $(25 \mathrm{~mL})$ was added $1 \mathrm{M} \mathrm{HCl}$ in $\mathrm{Et}_{2} \mathrm{O}(450 \mu \mathrm{L}, 1.1$ equiv) followed by the Grubbs II catalyst $(18 \mathrm{mg}, 0.021 \mathrm{mmol})$. The reaction mixture was heated to reflux for $16 \mathrm{~h}$, whereupon it was cooled to $20{ }^{\circ} \mathrm{C}$ and saturated aqueous $\mathrm{NaHCO}_{3}(20 \mathrm{~mL})$ was added under stirring. The organic layer was separated and the aqueous phase was extracted with $\mathrm{CH}_{2} \mathrm{Cl}_{2}(3 \times 10 \mathrm{~mL})$. The combined organic layers were dried over sodium sulfate and the volatiles were removed in vacuo. The residue was purified by flash column chromatography on silica gel (EtOAc/heptane, 1:4 and 3:4, $R_{\mathrm{f}}=$ 0.15 in EtOAc/heptane, 1:4) to give compound 18 as a clear yellow oil (155 mg, 86\%). ${ }^{1} \mathrm{H} \mathrm{NMR}\left(400 \mathrm{MHz}, \mathrm{CDCl}_{3}\right) \delta 7.41-7.23$ $(\mathrm{m}, 5 \mathrm{H}), 5.88(\mathrm{td}, \mathrm{J}=17.3,10.7 \mathrm{~Hz}, 1 \mathrm{H}), 5.75-5.60(\mathrm{~m}, 2 \mathrm{H}), 5.23$ $(\mathrm{dd}, J=17.3,1.4 \mathrm{~Hz}, 1 \mathrm{H}), 5.07(\mathrm{dd}, \mathrm{J}=10.9,3.0 \mathrm{~Hz}, 1 \mathrm{H}), 4.17$ (dd, $J=13.3,6.2 \mathrm{~Hz}, 1 \mathrm{H}), 3.99(\mathrm{q}, \mathrm{J}=3.9 \mathrm{~Hz}, 1 \mathrm{H}), 3.68-3.20$ $(\mathrm{m}, 4 \mathrm{H}), 3.19-3.00(\mathrm{~m}, 2 \mathrm{H}), 2.94(\mathrm{~s}, 3 \mathrm{H}), 2.52-2.29(\mathrm{~m}, 3 \mathrm{H})$, $2.28-1.92(\mathrm{~m}, 3 \mathrm{H}), 1.47-1.42\left(\mathrm{~m}, 9 \mathrm{H}\right.$, two rotamers). ${ }^{13} \mathrm{C}$ NMR $\left(101 \mathrm{MHz}, \mathrm{CDCl}_{3}\right) \delta 156.8$ (major rotamer), 155.9 (minor rotamer), 144.0 (major rotamer), 143.5 (minor rotamer), 138.7, 129.5 (major rotamer), 129.3 (minor rotamer), 128.9 (2C), 128.5 (2C), 127.4, 124.6 (minor rotamer), 124.4 (major rotamer), 113.8 (minor rotamer), 113.6 (major rotamer), 91.3 (major rotamer), 91.2 (minor rotamer), 88.6 (major rotamer), 88.1 (minor rotamer), 79.8 (minor rotamer), 79.5 (major rotamer), 70.7 (major rotamer), 70.6 (minor rotamer), 65.9 (minor rotamer), 65.6 (major rotamer), 59.2, 57.7 (minor rotamer), 57.2, 56.9 (major rotamer), 47.3 (major rotamer), 47.2 (minor rotamer), 37.8 (minor rotamer), 37.4 (major rotamer), 37.3 (major rotamer), 36.9 (minor rotamer), 28.7, 28.5
(3C). HRMS (ESI) calcd for $\mathrm{C}_{26} \mathrm{H}_{37} \mathrm{~N}_{2} \mathrm{O}_{4}\left[\mathrm{M}+\mathrm{H}^{+}\right] 441.2748$, found 441.2783 .

Tert-butyl

$\left(\left(\left(2 S^{*}, 3 a R^{*}, 5 a S^{\star}, 6 S^{*}, 9 a R^{\star}\right)-2-\right.\right.$ ethyl-6hydroxydecahydrofuro[3,2-c]indol-2-

yl)methyl)(methyl)carbamate (S7). General Method C was applied to tert-butyl $\left(\left(\left(2 R^{*}, 3 a R^{*}, 5 a S^{*}, 6 S^{*}, 9 a R^{*}\right)-5\right.\right.$-benzyl-6hydroxy-2-vinyl-2,3,3a,4,5,5a,6,7-octahydrofuro[3,2-c]indol-2yl)methyl)(methyl)carbamate (18) (905 mg, $2.05 \mathrm{mmol}$ ). The crude was purified by flash column chromatography on silica gel $\left(\mathrm{CH}_{2} \mathrm{Cl}_{2} / \mathrm{MeOH}\right.$ with $5 \%$ of $\mathrm{NH}_{4} \mathrm{OH}(25 \%$ aq $\left.) 9: 1, R_{\mathrm{f}}=0.1\right)$ and the desired compound $\mathbf{S 7}$ was isolated a colorless oil $(340 \mathrm{mg}$, $78 \%$ ) which was not completely pure but used in the next step without further purification.

$\left(2 S^{*}, 3 a R^{*}, 5 a S^{*}, 6 S^{*}, 9 a R^{*}\right)-2-e t h y l-2-(($ methylamino $)$ methyl)-5(pyridin-3-ylmethyl)decahydrofuro[3,2-c]indol-6-ol (S8). General Method D was applied to tert-butyl $\left(\left(\left(2 S^{*}, 3 a R^{*}, 5 a S^{*}, 6 S^{*}, 9 a R^{*}\right)-\right.\right.$ 2-ethyl-6-hydroxydecahydrofuro[3,2-c]indol-2-

$\mathrm{yl}$ )methyl)(methyl)carbamate (S7) (143 mg, $0.4 \mathrm{mmol}$ ) and nicotinaldehyde $(45 \mu \mathrm{L}, 0.48 \mathrm{mmol})$. The crude was then solubilized in a $3: 1$ mixture of $\mathrm{CH}_{2} \mathrm{Cl}_{2}$ and TFA $(0.1 \mathrm{M})$ and stirred for $1 \mathrm{~h}$ at $20^{\circ} \mathrm{C}$. Then, it was concentrated under reduced pressure and used directly in the next step without further purification.

3-(3-Chlorophenyl)-1-((2S*,3aR*,5aS*,6S*,9aR*)-2-ethyl-6hydroxy-5-(pyridin-3-ylmethyl)decahydrofuro[3,2-c]indol-2-

yl)methyl)-1-methylurea (19) General Method $\mathbf{G}$ was applied to $\left(2 S^{*}, 3 a R^{*}, 5 a S^{*}, 6 S^{*}, 9 a R^{*}\right)-2$-ethyl-2-((methylamino)methyl)-5(pyridin-3-ylmethyl)decahydrofuro[3,2-c]indol-6-ol (S8) (22.6 mg, $0.06 \mathrm{mmol}$ ) and 3-chlorophenyl isocyanate $(10 \mu \mathrm{L}, 0.065 \mathrm{mmol})$ to give the desired compound as an amorphous white solid (19.8 $\mathrm{mg}, 61 \%$ over three steps). ${ }^{1} \mathrm{H}$ NMR $\left(400 \mathrm{MHz}, \mathrm{CDCl}_{3}\right) \delta 8.70-$ $8.25(\mathrm{~m}, 3 \mathrm{H}), 7.79-7.55(\mathrm{~m}, 1 \mathrm{H}), 7.50(\mathrm{t}, \mathrm{J}=2.1 \mathrm{~Hz}, 1 \mathrm{H}), 7.30-$ $7.13(\mathrm{~m}, 3 \mathrm{H}), 7.01-6.89(\mathrm{~m}, 1 \mathrm{H}), 5.23-4.50(\mathrm{~m}, 2 \mathrm{H}), 4.46-$ $4.11(\mathrm{~m}, 1 \mathrm{H}), 4.11-3.78(\mathrm{~m}, 1 \mathrm{H}), 3.75-3.30(\mathrm{~m}, 2 \mathrm{H}), 3.30-$ $3.09(\mathrm{~m}, 1 \mathrm{H}), 3.05(\mathrm{~s}, 3 \mathrm{H}), 2.88-2.69(\mathrm{~m}, 1 \mathrm{H}), 2.50$ (br. s, $1 \mathrm{H})$, 2.32 (br. s, $1 \mathrm{H}), 2.08-1.86(\mathrm{~m}, 3 \mathrm{H}), 1.86-1.43(\mathrm{~m}, 7 \mathrm{H}), 0.95(\mathrm{t}$, $\mathrm{J}=7.5 \mathrm{~Hz}, 3 \mathrm{H}) ;{ }^{13} \mathrm{C}$ NMR $\left(101 \mathrm{MHz}, \mathrm{CDCl}_{3}\right) \delta 165.1,157.7,149.5$, $141.1,137.4,134.7,130.1,123.8,122.6,122.8,119.2,117.0$, 93.5, 90.6, 77.4, 70.5, 63.9, 58.1, 46.6, 46.4, 38.8, 38.6, 33.7, $32.7,27.0,18.5,8.9$. HRMS (ESI) calcd for $\mathrm{C}_{27} \mathrm{H}_{36} \mathrm{CIN}_{4} \mathrm{O}_{3}\left[\mathrm{M}+\mathrm{H}^{+}\right]$ 499.2476, found 499.2485

$\left(2 S^{*}, 3 a R^{*}, 5 a S^{*}, 6 S^{*}, 9 a R^{*}\right)-2-E$ thyl-2-((methylamino)methyl)-5pentyldecahydrofuro[3,2-c]indol-6-ol (S9). General Method E was applied to tert-butyl $\left(\left(\left(2 \mathrm{~S}^{*}, 3 \mathrm{aR}^{*}, 5 \mathrm{aS}^{*}, 6 \mathrm{~S}^{*}, 9 a \mathrm{R}^{\star}\right)\right.\right.$-2-ethyl-6hydroxydecahydrofuro[3,2-c]indol-2-

yl)methyl)(methyl)carbamate (S7) (134 mg, $0.38 \mathrm{mmol}$ ) and valeraldehyde $(48 \mu \mathrm{L}, 0.45 \mathrm{mmol})$. After the reaction, the crude mixture was dissolved in a $3: 1$ mixture of $\mathrm{CH}_{2} \mathrm{Cl}_{2}$ and TFA $(0.1 \mathrm{M})$ and stirred for $1 \mathrm{~h}$ at $20^{\circ} \mathrm{C}$. The reaction mixture was concentrated under reduced pressure and used directly in the next step without further purification.

$N-\left(\left(\left(2 S^{*}, 3 a R^{*}, 5 a S^{*}, 6 S^{*}, 9 a R^{*}\right)-2-E\right.\right.$ thyl-6-hydroxy-5pentyldecahydrofuro[3,2-c]indol-2-yl)methyl)-N-methylthiophene2-carboxamide (20). General Method $\mathbf{H}$ was applied to $\left(2 S^{*}, 3 a R^{*}, 5 a S^{\star}, 6 S^{*}, 9 a R^{\star}\right)-2$-Ethyl-2-((methylamino)methyl)-5pentyldecahydrofuro[3,2-c]indol-6-ol (S9) $(22.6 \mathrm{mg}, 0.07 \mathrm{mmol})$ and thiophene-2-carbonyl chloride $(8 \mu \mathrm{L}, 0.077 \mathrm{mmol})$ to give the desired compound as an amorphous white solid (18.1 mg, 60f \% over three steps). ${ }^{1} \mathrm{H}$ NMR $\left(400 \mathrm{MHz}, \mathrm{CDCl}_{3}\right) \delta 8.52(\mathrm{~s}, 1 \mathrm{H}), 7.52$ $(\mathrm{d}, J=5.0 \mathrm{~Hz}, 1 \mathrm{H}), 7.46(\mathrm{~d}, J=3.7 \mathrm{~Hz}, 1 \mathrm{H}), 7.09$ (dd, $J=5.1,3.8$ $\mathrm{Hz}, 1 \mathrm{H}), 6.92$ (br. s, $1 \mathrm{H}), 4.25$ (br. s, $1 \mathrm{H}), 4.13-3.51(\mathrm{~m}, 2 \mathrm{H})$, 
$3.51-3.31(\mathrm{~m}, 4 \mathrm{H}), 3.30-2.58(\mathrm{~m}, 4 \mathrm{H}), 2.13(\mathrm{dd}, \mathrm{J}=13.8,7.3$ $\mathrm{Hz}, 1 \mathrm{H}), 2.08-1.11(\mathrm{~m}, 15 \mathrm{H}), 0.97(\mathrm{t}, \mathrm{J}=7.5 \mathrm{~Hz}, 3 \mathrm{H}), 0.93-0.84$ $(\mathrm{m}, 3 \mathrm{H}) ;{ }^{13} \mathrm{C}$ NMR $\left(101 \mathrm{MHz}, \mathrm{CDCl}_{3}\right) \delta 167.8,166.0,137.7,130.5$, $127.3,92.2,88.2,77.4,64.9,59.0,54.2,45.4,41.2,39.8,33.4$, $33.1,29.6,29.0,25.6,22.3,17.3,14.0,9.2,1.1$. HRMS (ESI) calcd for $\mathrm{C}_{24} \mathrm{H}_{39} \mathrm{~N}_{2} \mathrm{O}_{3} \mathrm{~S}\left[\mathrm{M}+\mathrm{H}^{+}\right]$435.2676, found 435.2702.

1'-(Tert-butyl) 6-methyl $\left(2 R^{*}, 3 a R^{*}, 6 R^{*}, 6 a R^{*}\right)-5$-benzyl-6a-vinyl3,3a,4,5,6,6a-hexahydro-2'H-spiro[furo[2,3-c]pyrrole-2,3'pyridine]-1',6(6'H)-dicarboxylate (21). Methyl $\left(3 R^{\star}, 3 a R^{\star}, 6 R^{\star}, 7 a R^{\star}\right)-6-(($ allyl $($ tert-

butoxycarbonyl)amino)methyl)-2-benzyl-1,2,3,6,7,7a-hexahydro3a,6-epoxyisoindole-3-carboxylate (7) $(5.0 \mathrm{~g}, 11 \mathrm{mmol})$ was dissolved in $\mathrm{CH}_{2} \mathrm{Cl}_{2}(40 \mathrm{~mL})$ and added $4 \mathrm{M} \mathrm{HCl}$ in dioxane (3.3 $\mathrm{mL}, 13.2 \mathrm{mmol}$ ) whereupon the mixture was concentrated in vacuo followed by 30 min under vacuum using an oil pump which gave a white $\mathrm{HCl}$-salt. The salt was then added dry $\mathrm{CH}_{2} \mathrm{Cl}_{2}(55$ $\mathrm{mL}$ ) followed by Grubbs II (93 mg, 1\%) and the atmosphere was exchanged to ethylene and the mixture was stirred at $20^{\circ} \mathrm{C}$. After $16 \mathrm{~h}$ Grubbs II (93 mg, 1\%) was added and the mixture was stirred $5 \mathrm{~h}$ at reflux under an ethylene atmosphere. The reaction was allowed to cool to $20^{\circ} \mathrm{C}$, added sat. $\mathrm{NaHCO}_{3}$ (aq.) $(100 \mathrm{~mL}$, pH > 7) and $\mathrm{CH}_{2} \mathrm{Cl}_{2}(50 \mathrm{~mL})$ and the layers were separated. The aqueous phase was extracted with $\mathrm{CH}_{2} \mathrm{Cl}_{2}(2 \times 50 \mathrm{~mL})$ and the combined organic layers were dried over $\mathrm{Na}_{2} \mathrm{SO}_{4}$ and concentrated in vacuo. The residue was purified by flash column chromatography (EtOAc:heptane 1:4, $R_{\mathrm{f}}=0.2$ ) to give the title compound as a white solid $(4.14 \mathrm{~g}, 83 \%)$. ${ }^{1} \mathrm{H}$ NMR $(400 \mathrm{MHz}$, $\left.\mathrm{CDCl}_{3}\right) \delta 7.42-7.13(\mathrm{~m}, 5 \mathrm{H}), 5.88(\mathrm{dd}, \mathrm{J}=16.9,10.6 \mathrm{~Hz}, 1 \mathrm{H})$, $5.71(\mathrm{~s}, 2 \mathrm{H}), 5.44(\mathrm{~d}, \mathrm{~J}=16.9 \mathrm{~Hz}, 1 \mathrm{H}), 5.11(\mathrm{~d}, \mathrm{~J}=10.6 \mathrm{~Hz}, 1 \mathrm{H})$, $4.37(\mathrm{~d}, \mathrm{~J}=13.1 \mathrm{~Hz}, 1 \mathrm{H}), 4.18(\mathrm{~d}, \mathrm{~J}=18.5 \mathrm{~Hz}, 1 \mathrm{H}), 3.81(\mathrm{~d}, \mathrm{~J}=$ $13.1 \mathrm{~Hz}, 1 \mathrm{H}), 3.72-3.49(\mathrm{~m}, 6 \mathrm{H}), 3.44(\mathrm{t}, \mathrm{J}=9.9 \mathrm{~Hz}, 1 \mathrm{H}), 3.28$ $3.05(\mathrm{~m}, 1 \mathrm{H}), 3.05-2.79(\mathrm{~m}, 2 \mathrm{H}), 2.01(\mathrm{~d}, \mathrm{~J}=13.4 \mathrm{~Hz}, 1 \mathrm{H}), 1.88$ $(\mathrm{dd}, \mathrm{J}=13.4,8.0 \mathrm{~Hz}, 1 \mathrm{H}), 1.52-1.36(\mathrm{~m}, 9 \mathrm{H}) ;{ }^{13} \mathrm{C}$ NMR $(101$ $\left.\mathrm{MHz}, \mathrm{CDCl}_{3}\right) \delta 171.6,154.6,138.4,133.1,128.9(2 \mathrm{C}), 128.5(2 \mathrm{C})$, $127.4,127.2,125.1,114.9,96.3,83.0,80.1,76.1,58.6,56.2$, 51.4, 50.6, 47.9, 42.7, 39.3, 28.6 (major rotamer, 3C). HRMS (ESI) calcd for $\mathrm{C}_{26} \mathrm{H}_{35} \mathrm{~N}_{2} \mathrm{O}_{5}\left[\mathrm{M}+\mathrm{H}^{+}\right] 455.2540$, found 455.2572

Tert-butyl $\quad\left(2 R^{*}, 3 a R^{*}, 6 R^{*}, 6 a R^{*}\right)-5-$ benzyl-6-(pyrrolidine-1carbonyl)-6a-vinyl-3,3a,4,5,6,6a-hexahydro-2'H-spiro[furo[2,3c]pyrrole-2,3'-pyridine]-1'(6'H)-carboxylate (22). General Method I was applied to $1^{\prime}$-(tert-butyl) 6 -methyl $\left(2 R^{\star}, 3 a R^{\star}, 6 R^{\star}, 6 a R^{\star}\right)-5$ benzyl-6a-vinyl-3,3a,4,5,6,6a-hexahydro-2'H-spiro[furo[2,3c]pyrrole-2,3'-pyridine]-1',6(6'H)-dicarboxylate (21) $(3.17 \mathrm{~g}, 6.96$ $\mathrm{mmol})$. General Method $\mathbf{J}$ was applied to the crude carboxylic acid $(1.53 \mathrm{~g}, 3.47 \mathrm{mmol})$ and pyrrolidine $(0.57 \mathrm{~mL}, 6.96 \mathrm{mmol})$. The crude mixture was purified by flash column chromatography (EtOAc:heptane 1:1, $R_{\mathrm{f}}=0.2$ ) to give the title compound as a white solid $(1.476 \mathrm{~g}, 86 \%) .{ }^{1} \mathrm{H}$ NMR $\left(400 \mathrm{MHz}, \mathrm{CDCl}_{3}\right) \delta 7.41-$ $7.16(\mathrm{~m}, 5 \mathrm{H}), 5.93(\mathrm{dd}, \mathrm{J}=16.9,10.6 \mathrm{~Hz}, 1 \mathrm{H}), 5.80-5.66(\mathrm{~m}$, 2H), $5.47(\mathrm{~d}, \mathrm{~J}=16.7 \mathrm{~Hz}, 1 \mathrm{H}), 5.05(\mathrm{~d}, \mathrm{~J}=10.5 \mathrm{~Hz}, 1 \mathrm{H}), 4.70-$ $3.69(\mathrm{~m}, 4 \mathrm{H}), 3.69-3.30(\mathrm{~m}, 5 \mathrm{H}), 3.21-2.86(\mathrm{~m}, 4 \mathrm{H}), 2.47-$ $2.20(\mathrm{~m}, 1 \mathrm{H}), 2.11-1.97(\mathrm{~m}, 1 \mathrm{H}), 1.88(\mathrm{dd}, J=13.3,8.4 \mathrm{~Hz}, 1 \mathrm{H})$, $1.76-1.54(\mathrm{~m}, 4 \mathrm{H}), 1.47(\mathrm{~s}, 9 \mathrm{H}) ;{ }^{13} \mathrm{C} \mathrm{NMR}\left(101 \mathrm{MHz}, \mathrm{CDCl}_{3}\right) \delta$ $169.5,154.8,139.3,139.1,133.2,129.0,128.4$ (2C), $127.2(2 \mathrm{C})$, $125.1,114.4,96.8,82.5,80.1,72.3,60.3,55.1,50.2,48.7,46.4$, 45.4, 42.7, 39.8, 28.7 (3C), 26.1, 24.2. HRMS (ESI) calcd for $\mathrm{C}_{29} \mathrm{H}_{40} \mathrm{~N}_{3} \mathrm{O}_{4}\left[\mathrm{M}+\mathrm{H}^{+}\right]$494.3013, found 494.3051.

Tert-butyl $\quad\left(2 R^{\star}, 3 a R^{*}, 6 R^{*}, 6 a R^{*}\right)-5$-benzyl-6(isopropylcarbamoyl)-6a-vinyl-3,3a,4,5,6,6a-hexahydro-2'Hspiro[furo[2,3-c]pyrrole-2,3'-pyridine]-1'(6'H)-carboxylate General Method I was applied to 1'-(tert-butyl) 6-methyl $\left(2 R^{\star}, 3 a R^{\star}, 6 R^{\star}, 6 a R^{\star}\right)-5$-benzyl-6a-vinyl-3,3a,4,5,6,6ahexahydro-2'H-spiro[furo[2,3-c]pyrrole-2,3'-pyridine]-1',6(6'H)- dicarboxylate (21) (3.166 g, $6.96 \mathrm{mmol})$. General Method J was applied to the crude carboxylic acid $(1.53 \mathrm{~g}, 3.47 \mathrm{mmol})$ and $i$ $\mathrm{PrNH}_{2}(0.60 \mathrm{~mL}, 6.96 \mathrm{mmol})$. The crude mixture was purified via flash column chromatography (EtOAc:heptane 2:3, $R_{\mathrm{f}}=0.2$ ) and the title compound was afforded as a white solid $(1.515 \mathrm{~g}, 91 \%)$. ${ }^{1} \mathrm{H}$ NMR $\left(400 \mathrm{MHz}, \mathrm{CDCl}_{3}\right) \delta 7.39-7.16(\mathrm{~m}, 5 \mathrm{H}), 6.68-6.49(\mathrm{~m}$, $1 \mathrm{H}), 6.03-5.87(\mathrm{~m}, 1 \mathrm{H}), 5.84-5.68(\mathrm{~m}, 1 \mathrm{H}), 5.68-5.49(\mathrm{~m}, 1 \mathrm{H})$, $5.37-5.17(\mathrm{~m}, 1 \mathrm{H}), 5.08(\mathrm{~d}, \mathrm{~J}=10.9 \mathrm{~Hz}, 1 \mathrm{H}), 4.24-3.80(\mathrm{~m}$, $4 \mathrm{H}), 3.80-3.47(\mathrm{~m}, 1 \mathrm{H}), 3.43-3.31(\mathrm{~m}, 2 \mathrm{H}), 3.25(\mathrm{t}, \mathrm{J}=8.4 \mathrm{~Hz}$, $1 \mathrm{H}), 3.16(\mathrm{~d}, J=12.8 \mathrm{~Hz}, 1 \mathrm{H}), 2.81(\mathrm{q}, J=8.2 \mathrm{~Hz}, 1 \mathrm{H}), 2.63(\mathrm{t}, J$ $=9.1 \mathrm{~Hz}, 1 \mathrm{H}), 2.07-1.90(\mathrm{~m}, 1 \mathrm{H}), 1.90-1.76(\mathrm{~m}, 1 \mathrm{H}), 1.46(\mathrm{~s}$, $9 \mathrm{H}), 1.14-1.06(\mathrm{~m}, 6 \mathrm{H}) .{ }^{13} \mathrm{C} \mathrm{NMR}\left(101 \mathrm{MHz}, \mathrm{CDCl}_{3}\right) \delta 169.0$, $154.4,138.6,138.1,133.4,128.8,128.6$ (2C), 127.5 (2C), 124.1, 114.0, 96.3, 82.5, 80.0, 79.0, 58.5, 57.9, 51.3, 47.1, 42.5, 40.6, 37.5, 28.6 (2C), 22.9 (major rotamer, 3C). HRMS (ESI) calcd for $\mathrm{C}_{28} \mathrm{H}_{40} \mathrm{~N}_{3} \mathrm{O}_{4}\left[\mathrm{M}+\mathrm{H}^{+}\right]$482.3013, found 482.3044 .

Methyl $\left(2 S^{*}, 3 a R^{\star}, 6 R^{*}, 6 a R^{*}\right)$-6a-ethyl-1'-pentyl-5-(thiophene-2carbonyl)hexahydrospiro[furo[2,3-c]pyrrole-2,3'-piperidine]-6carboxylate (24). General Method F was applied to 1'-(tert-butyl) 6-methyl $\left(2 R^{\star}, 3 a R^{\star}, 6 R^{\star}, 6 a R^{\star}\right)-5$-benzyl-6a-vinyl-3,3a,4,5,6,6ahexahydro-2'H-spiro[furo[2,3-c]pyrrole-2,3'-pyridine]-1',6(6'H)dicarboxylate $(21)(207 \mathrm{mg}, 0.445 \mathrm{mmol})$ followed by General Method E with valeraldehyde $(63 \mu \mathrm{L}, 0.592 \mathrm{mmol})$, General Method B, and General Method H with thiophene-2-carbonyl chloride $(58 \mu \mathrm{L}, 0.456 \mathrm{mmol})$, respectively. The crude mixture was purified directly using flash column chromatography (EtOAc:heptane $9: 1+1 \% \mathrm{Et}_{3} \mathrm{~N}, R_{\mathrm{f}}=0.2$ ) to give the title compound as a yellow oil (158 mg, 77\%). ${ }^{1} \mathrm{H}$ NMR $(400 \mathrm{MHz}$, $\left.\mathrm{CDCl}_{3}\right) \delta 7.48(\mathrm{~d}, \mathrm{~J}=3.4 \mathrm{~Hz}, 1 \mathrm{H}), 7.43(\mathrm{~d}, \mathrm{~J}=4.8 \mathrm{~Hz}, 1 \mathrm{H}), 7.04-$ $6.98(\mathrm{~m}, 1 \mathrm{H}), 4.85(\mathrm{~s}, 1 \mathrm{H}), 4.22-3.85(\mathrm{~m}, 2 \mathrm{H}), 3.67(\mathrm{~s}, 3 \mathrm{H}), 2.80$ $(\mathrm{d}, \mathrm{J}=4.6 \mathrm{~Hz}, 1 \mathrm{H}), 2.43(\mathrm{t}, \mathrm{J}=11.4 \mathrm{~Hz}, 2 \mathrm{H}), 2.32-2.04(\mathrm{~m}, 3 \mathrm{H})$, $2.04-1.96(\mathrm{~m}, 2 \mathrm{H}), 1.69-1.59(\mathrm{~m}, 1 \mathrm{H}), 1.60-1.31(\mathrm{~m}, 5 \mathrm{H})$, $1.32-1.07(\mathrm{~m}, 6 \mathrm{H}), 0.95(\mathrm{t}, \mathrm{J}=7.3 \mathrm{~Hz}, 3 \mathrm{H}), 0.85-0.72(\mathrm{~m}, 4 \mathrm{H})$ (two rotamers). ${ }^{13} \mathrm{C}$ NMR $\left(101 \mathrm{MHz}, \mathrm{CDCl}_{3}\right) \delta 170.9,162.2,137.8$, 130.5, 130.3, 127.1, 92.7, 83.5, 71.0, 64.0, 58.6, 54.4, 53.7, 52.0, 46.0, 41.2, 37.6, 29.7, 27.2, 26.3, 23.4, 22.4, 14.1, 8.3 (major rotamer). HRMS (ESI) calcd for $\mathrm{C}_{24} \mathrm{H}_{37} \mathrm{~N}_{2} \mathrm{O}_{4} \mathrm{~S}\left[\mathrm{M}+\mathrm{H}^{+}\right] 449.2469$, found 449.2525 .

$\left(\left(2 S^{*}, 3 a R^{*}, 6 R^{*}, 6 a R^{*}\right)-6 a-E t h y l-1 '-p e n t y l-5-(t h i o p h e n e-2-\right.$ carbonyl)hexahydrospiro[furo[2,3-c]pyrrole-2,3'-piperidin]-6$y$ l)(morpholino)methanone (25). General Method I was applied to methyl $\left(2 S^{*}, 3 a R^{\star}, 6 R^{\star}, 6 a R^{\star}\right)$-6a-ethyl-1'-pentyl-5-(thiophene-2carbonyl)hexahydrospiro[furo[2,3-c]pyrrole-2,3'-piperidine]-6carboxylate $(24)(158 \mathrm{mg}, 0.352 \mathrm{mmol})$. The carboxylic acid $(20$ $\mathrm{mg}, 0.046 \mathrm{mmol})$ and morpholine ( $8 \mu \mathrm{L}, 0.092)$ was used following General Method J. The mixture was purified directly using preparative HPLC to give the title compound as a white solid (14 $\mathrm{mg}, 60 \%) .{ }^{1} \mathrm{H}$ NMR $\left(400 \mathrm{MHz}, \mathrm{CDCl}_{3}\right) \delta 7.55(\mathrm{~d}, \mathrm{~J}=3.8 \mathrm{~Hz}, 1 \mathrm{H})$, 7.49 (dd, $J=5.0,0.8 \mathrm{~Hz}, 1 \mathrm{H}), 7.08(\mathrm{dd}, \mathrm{J}=5.0,3.9 \mathrm{~Hz}, 1 \mathrm{H}), 5.33$ (s, $1 \mathrm{H}), 4.37$ (dd, J = 10.5, 8.9 Hz, 1H), 3.95 (dd, J = 10.7, 3.0 Hz, $2 \mathrm{H}), 3.87-3.76(\mathrm{~m}, 1 \mathrm{H}), 3.77-3.65(\mathrm{~m}, 5 \mathrm{H}), 3.50$ (ddd, $J=13.0$, $6.7,3.5 \mathrm{~Hz}, 1 \mathrm{H}), 3.10-2.99(\mathrm{~m}, 1 \mathrm{H}), 2.85-2.76(\mathrm{~m}, 1 \mathrm{H}), 2.64$ $(\mathrm{d}, \mathrm{J}=11.3 \mathrm{~Hz}, 1 \mathrm{H}), 2.51-2.32(\mathrm{~m}, 4 \mathrm{H}), 2.26(\mathrm{~d}, \mathrm{~J}=11.9 \mathrm{~Hz}$, $1 \mathrm{H}), 2.05(\mathrm{dd}, \mathrm{J}=13.6,8.1 \mathrm{~Hz}, 1 \mathrm{H}), 1.92-1.81(\mathrm{~m}, 1 \mathrm{H}), 1.78-$ $1.67(\mathrm{~m}, 1 \mathrm{H}), 1.67-1.56(\mathrm{~m}, 4 \mathrm{H}), 1.33(\mathrm{dt}, \mathrm{J}=14.1,7.1 \mathrm{~Hz}, 2 \mathrm{H})$, $1.22-1.10(\mathrm{~m}, 4 \mathrm{H}), 0.97(\mathrm{t}, J=7.4 \mathrm{~Hz}, 3 \mathrm{H}), 0.82(\mathrm{t}, J=6.9 \mathrm{~Hz}$, $3 \mathrm{H}) ;{ }^{13} \mathrm{C}$ NMR $\left(101 \mathrm{MHz}, \mathrm{CDCl}_{3}\right) \delta 168.9,167.3,138.0,130.8$, $130.5,127.5,94.7,82.4,67.1,67.0,66.7,61.7,58.0,55.7,53.1$, $47.3,47.1,42.5,41.8,37.5,29.5,27.5,25.0,22.3,22.1,14.1,9.0$. HRMS (ESI) calcd for $\mathrm{C}_{27} \mathrm{H}_{42} \mathrm{~N}_{3} \mathrm{O}_{4} \mathrm{~S}\left[\mathrm{M}+\mathrm{H}^{+}\right] 504.2891$, found 504.2955 . 
Tert-butyl $\left(2 S^{*}, 3 a R^{*}, 6 R^{*}, 6 a R^{*}\right)-6 a-e t h y l-5$-(pyridin-2-ylmethyl)-6(pyrrolidine-1-carbonyl)hexahydrospiro[furo[2,3-c]pyrrole-2,3'piperidine]-1'-carboxylate (26). General Method B was applied to tert-butyl $\quad\left(2 R^{*}, 3 a R^{*}, 6 R^{*}, 6 a R^{*}\right)$-5-benzyl-6-(pyrrolidine-1carbonyl)-6a-vinyl-3,3a,4,5,6,6a-hexahydro-2'H-spiro[furo[2,3c]pyrrole-2,3'-pyridine]-1'(6'H)-carboxylate (22) (1.32g, 2.65 $\mathrm{mmol})$. Following this reaction, General Method E was applied to the amine (567 mg, $1.39 \mathrm{mmol}$ ) and 2-pyridinecarboxaldehyde $(164 \mu \mathrm{L}, 1.74 \mathrm{mmol})$. The crude mixture was purified directly using flash column chromatography (EtOAc:MeOH 9:2, $R_{\mathrm{f}}=0.2$ ) to give the title compound as a yellow solid $(697 \mathrm{mg}, 95 \%) .{ }^{1} \mathrm{H}$ NMR $\left(400 \mathrm{MHz}, \mathrm{CDCl}_{3}\right) \delta 8.46(\mathrm{~d}, \mathrm{~J}=4.9 \mathrm{~Hz}, 1 \mathrm{H}), 7.75-7.53(\mathrm{~m}$, $2 \mathrm{H}), 7.16-7.09(\mathrm{~m}, 1 \mathrm{H}), 4.48-3.90(\mathrm{~m}, 2 \mathrm{H}), 3.85-3.56(\mathrm{~m}, 3 \mathrm{H})$, $3.56-3.23(\mathrm{~m}, 4 \mathrm{H}), 3.10-2.82(\mathrm{~m}, 2 \mathrm{H}), 2.81-2.59(\mathrm{~m}, 2 \mathrm{H})$, $2.04-1.89(\mathrm{~m}, 1 \mathrm{H}), 1.81-1.50(\mathrm{~m}, 11 \mathrm{H}), 1.43(\mathrm{~s}, 10 \mathrm{H}), 0.84(\mathrm{t}$, $\mathrm{J}=7.3 \mathrm{~Hz}, 3 \mathrm{H}) .{ }^{13} \mathrm{C} \mathrm{NMR}\left(101 \mathrm{MHz}, \mathrm{CDCl}_{3}\right) \delta 174.4,169.3,159.6$, 154.9, 148.6, 136.8, 122.1, 97.8, 83.7, 79.9, 73.4, 61.2, 57.8, 53.5, 46.5, 45.6, 43.2, 40.2, 39.1, 29.3 (3C), 28.7, 26.4, 24.1, 23.7, 21.6, 8.7. HRMS (ESI) calcd for $\mathrm{C}_{28} \mathrm{H}_{43} \mathrm{~N}_{4} \mathrm{O}_{4}\left[\mathrm{M}+\mathrm{H}^{+}\right]$ 499.3279, found 499.3376 .

((2S*,3aR*,6R*,6aR*)-1'-(Butylsulfonyl)-6a-ethyl-5-(pyridin-2ylmethyl)hexahydrospiro[furo[2,3-c]pyrrole-2,3'-piperidin]-6yl)(pyrrolidin-1-yl)methanone (27). General Method F was applied to tert-butyl $\left(2 S^{\star}, 3 a R^{*}, 6 R^{\star}, 6 a R^{*}\right)$-6a-ethyl-5-(pyridin-2ylmethyl)-6-(pyrrolidine-1-carbonyl)hexahydrospiro[furo[2,3c]pyrrole-2,3'-piperidine]-1'-carboxylate (26) (85 mg, $0.17 \mathrm{mmol}$ ). Following this reaction, General Method $\mathbf{K}$ was applied to TFA amine salt (32 mg, $0.051 \mathrm{mmol}$ ) and 1-butanesulfonyl chloride (6 $\mathrm{mg}, 0.038 \mathrm{mmol}$ ) to give after preparative HPLC, the title compound as a white foam $(25 \mathrm{mg}, 93 \%) .{ }^{1} \mathrm{H}$ NMR $(400 \mathrm{MHz}$, $\left.\mathrm{CDCl}_{3}\right) \delta 8.49(\mathrm{dd}, J=4.9,0.9 \mathrm{~Hz}, 1 \mathrm{H}), 7.64(\mathrm{td}, J=7.6,1.8 \mathrm{~Hz}$, $1 \mathrm{H}), 7.52(\mathrm{~d}, J=7.8 \mathrm{~Hz}, 1 \mathrm{H}), 7.13$ (ddd, $J=7.3,4.9,1.0 \mathrm{~Hz}, 1 \mathrm{H}$ ), $3.97(\mathrm{~d}, \mathrm{~J}=13.8 \mathrm{~Hz}, 1 \mathrm{H}), 3.82(\mathrm{~d}, \mathrm{~J}=11.0 \mathrm{~Hz}, 1 \mathrm{H}), 3.81(\mathrm{~s}, 1 \mathrm{H})$, $3.73(\mathrm{~d}, \mathrm{~J}=13.8 \mathrm{~Hz}, 1 \mathrm{H}), 3.62-3.51(\mathrm{~m}, 2 \mathrm{H}), 3.46-3.36(\mathrm{~m}$, $3 \mathrm{H}), 3.14-3.04(\mathrm{~m}, 2 \mathrm{H}), 3.03-2.94(\mathrm{~m}, 1 \mathrm{H}), 2.93-2.86(\mathrm{~m}$, $2 \mathrm{H}), 2.83(\mathrm{dd}, \mathrm{J}=9.0,4.7 \mathrm{~Hz}, 1 \mathrm{H}), 2.79-2.72(\mathrm{~m}, 1 \mathrm{H}), 2.12(\mathrm{~d}, \mathrm{~J}$ $=13.4 \mathrm{~Hz}, 1 \mathrm{H}), 1.88-1.73(\mathrm{~m}, 8 \mathrm{H}), 1.68-1.54(\mathrm{~m}, 5 \mathrm{H}), 1.49-$ $1.38(\mathrm{~m}, 2 \mathrm{H}), 0.94(\mathrm{t}, J=7.4 \mathrm{~Hz}, 3 \mathrm{H}), 0.87(\mathrm{t}, J=7.3 \mathrm{~Hz}, 3 \mathrm{H}) ;{ }^{13} \mathrm{C}$ NMR $\left(101 \mathrm{MHz}, \mathrm{CDCl}_{3}\right) \delta 169.4,159.1,148.8,136.7,123.9$, $122.1,98.2$, 83.0, 73.6, 60.5, 57.7, 54.8, 49.6, 46.9, 46.3, 45.9, $45.7,41.2,38.1,29.1,26.4,25.3,24.2,23.8,22.0,13.8,8.8$ HRMS (ESI) calcd for $\mathrm{C}_{27} \mathrm{H}_{43} \mathrm{~N}_{4} \mathrm{O}_{4} \mathrm{~S}\left[\mathrm{M}+\mathrm{H}^{+}\right] 519.3000$, found 519.3035 .

$\left(2 R^{*}, 3 a R^{*}, 6 R^{*}, 6 a R^{*}\right)-1^{\prime}-$ Benzoyl-5-benzyl-N-isopropyl-6a-vinyl$1^{\prime}, 3,3 a, 4,5,6,6 a, 6^{\prime}$-octahydro-2' $\mathrm{H}$-spiro[furo[2,3-c]pyrrole-2, 3'pyridine]-6-carboxamide (28). General Method $\mathbf{F}$ was applied to tert-butyl $\left(2 R^{\star}, 3 a R^{\star}, 6 R^{*}, 6 a R^{*}\right)-5$-benzyl-6-(isopropylcarbamoyl)6a-vinyl-3,3a,4,5,6,6a-hexahydro-2'H-spiro[furo[2,3-c]pyrrole-

2,3'-pyridine]-1'(6'H)-carboxylate (23) (1.439 g, $2.99 \mathrm{mmol})$. General Method H was applied to amine $(570 \mathrm{mg}, 1.49 \mathrm{mmol})$ and benzoyl chloride $(208 \mu \mathrm{L}, 1.79 \mathrm{mmol})$. The mixture was purified using flash column chromatography (gradient from EtOAc:heptane $3: 2$ to $100 \%$ EtOAc, $R_{\mathrm{f}}=0.3$ in $100 \%$ EtOAc) to give the title compound as a white solid $(616 \mathrm{mg}, 85 \%) .{ }^{1} \mathrm{H}$ NMR $\left(400 \mathrm{MHz}, \mathrm{CDCl}_{3}\right)$ ठ $7.64-7.35(\mathrm{~m}, 5 \mathrm{H}), 7.37-7.20(\mathrm{~m}, 4 \mathrm{H}), 7.18$ $-6.99(\mathrm{~m}, 1 \mathrm{H}), 6.72(\mathrm{~s}, 0.5 \mathrm{H}), 6.42(\mathrm{~d}, \mathrm{~J}=6.5 \mathrm{~Hz}, 0.5 \mathrm{H}), 6.07-$ $5.46(\mathrm{~m}, 3 \mathrm{H}), 5.25(\mathrm{~d}, \mathrm{~J}=16.9 \mathrm{~Hz}, 1 \mathrm{H}), 5.08(\mathrm{~d}, \mathrm{~J}=10.9 \mathrm{~Hz}, 1 \mathrm{H})$, $4.65(\mathrm{~d}, \mathrm{~J}=11.0 \mathrm{~Hz}, 0.5 \mathrm{H}), 4.32-3.76(\mathrm{~m}, 4 \mathrm{H}), 3.80-3.59(\mathrm{~m}$, $2 \mathrm{H}), 3.55-3.07(\mathrm{~m}, 2 \mathrm{H}), 3.12-2.62(\mathrm{~m}, 2.5 \mathrm{H}), 1.95-1.77(\mathrm{~m}$, $1 \mathrm{H}), 1.79-1.54(\mathrm{~m}, 1 \mathrm{H}), 1.19-0.96(\mathrm{~m}, 6 \mathrm{H})$ (two rotamers). ${ }^{13} \mathrm{C}$ NMR $\left(101 \mathrm{MHz}, \mathrm{CDCl}_{3}\right) \delta 171.1,168.9,138.6,138.1,136.2$, 134.3, 132.6, 129.7, 128.7 (2C), 128.6 (2C), 127.8 (2C), 127.5 (2C), 124.6, 114.1, 96.4, 79.0, 60.5, 58.8, 58.2, 54.9, 46.7, 41.5,
40.6, 38.1, 22.9, 14.3 (major rotamer). HRMS (ESI) calcd for $\mathrm{C}_{30} \mathrm{H}_{36} \mathrm{~N}_{3} \mathrm{O}_{3}\left[\mathrm{M}+\mathrm{H}^{+}\right]$486.2751, found 486.2754.

$\left(2 S^{*}, 3 a R^{*}, 6 R^{*}, 6 a R^{*}\right)-1^{\prime}-$ Benzoyl-6a-ethyl-Nisopropylhexahydrospiro[furo[2,3-c]pyrrole-2,3'-piperidine]-6carboxamide (S10). $\left(2 R^{*}, 3 a R^{*}, 6 R^{*}, 6 a R^{\star}\right)-1^{\prime}$-benzoyl-5-benzyl-Nisopropyl-6a-vinyl-1',3,3a,4,5,6,6a,6'-octahydro-2' $\mathrm{H}$ spiro[furo[2,3-c]pyrrole-2,3'-pyridine]-6-carboxamide (28) (318 $\mathrm{mg}, 0.65 \mathrm{mmol}$ ) was hydrogenated following General Method B to give the title compound as a white solid (245 mg, 95\%). The amine was used directly without further purification.

$\left(2 S^{*}, 3 a R^{*}, 6 R^{*}, 6 a R^{\star}\right)-1^{\prime}-$ Benzoyl-5-(cyclopropylsulfonyl)-6aethyl- $N$-isopropylhexahydrospiro[furo[2,3-c]pyrrole-2,3'piperidine]-6-carboxamide (29). General Method $\mathbf{K}$ was applied to $\quad\left(2 S^{*}, 3 a R^{*}, 6 R^{*}, 6 a R^{*}\right)-1^{\prime}$-benzoyl-6a-ethyl- $N$ isopropylhexahydrospiro[furo[2,3-c]pyrrole-2,3'-piperidine]-6carboxamide (S10) (18 $\mathrm{mg}, \quad 0.045 \mathrm{mmol})$ and cyclopropanesulfonyl chloride $(7.6 \mathrm{mg}, 0.054 \mathrm{mmol})$ gave after preparative HPLC the title compound as a white foam $(17 \mathrm{mg}$, $75 \%)$. ${ }^{1} \mathrm{H} \mathrm{NMR}\left(400 \mathrm{MHz}, \mathrm{CDCl}_{3}\right) \delta 7.43-7.39(\mathrm{~m}, 3 \mathrm{H}), 7.36(\mathrm{~d}$, $\mathrm{J}=4.6 \mathrm{~Hz}, 2 \mathrm{H}), 6.94(\mathrm{~d}, \mathrm{~J}=7.2 \mathrm{~Hz}, 1 \mathrm{H}), 4.44(\mathrm{~s}, 1 \mathrm{H}), 4.41(\mathrm{~s}$, $1 \mathrm{H}), 4.07$ (dq, $J=13.3,6.6 \mathrm{~Hz}, 1 \mathrm{H}), 3.75(\mathrm{~d}, J=10.3 \mathrm{~Hz}, 1 \mathrm{H})$, $3.58(\mathrm{dd}, J=10.4,6.3 \mathrm{~Hz}, 1 \mathrm{H}), 3.52(\mathrm{~d}, J=13.5 \mathrm{~Hz}, 1 \mathrm{H}), 3.22(\mathrm{~d}$, $\mathrm{J}=13.1 \mathrm{~Hz}, 1 \mathrm{H}), 3.14(\mathrm{t}, \mathrm{J}=11.9 \mathrm{~Hz}, 1 \mathrm{H}), 2.91-2.83(\mathrm{~m}, 1 \mathrm{H})$, $2.76(\mathrm{dd}, \mathrm{J}=14.7,6.8 \mathrm{~Hz}, 1 \mathrm{H}), 2.19-1.94(\mathrm{~m}, 2 \mathrm{H}), 1.93-1.73$ $(\mathrm{m}, 3 \mathrm{H}), 1.67(\mathrm{q}, \mathrm{J}=7.2 \mathrm{~Hz}, 2 \mathrm{H}), 1.57(\mathrm{t}, \mathrm{J}=11.8 \mathrm{~Hz}, 1 \mathrm{H}), 1.49-$ $1.38(\mathrm{~m}, 1 \mathrm{H}), 1.13(\mathrm{~d}, \mathrm{~J}=6.5 \mathrm{~Hz}, 3 \mathrm{H}), 1.08(\mathrm{~d}, \mathrm{~J}=6.5 \mathrm{~Hz}, 3 \mathrm{H})$, 0.94 (t, $J=7.3 \mathrm{~Hz}, 6 \mathrm{H}) .{ }^{13} \mathrm{C}$ NMR $\left(101 \mathrm{MHz}, \mathrm{CDCl}_{3}\right) \delta 171.3$, 168.3, 136.6, 129.6, 128.6 (2C), 126.5 (2C), 95.2, 82.1, 70.5, $52.9,50.5,47.9,43.6,41.5,40.0,37.5,29.8,25.0,23.1,22.8$, $22.5,8.3,5.4,5.3$. HRMS (ESI) calcd for $\mathrm{C}_{26} \mathrm{H}_{38} \mathrm{~N}_{3} \mathrm{O}_{5} \mathrm{~S}\left[\mathrm{M}+\mathrm{H}^{+}\right]$ 504.2527, found 504.2539.

$\left(2 S^{*}, 3 a R^{\star}, 6 R^{\star}, 6 a R^{*}\right)-6 a-E t h y l-1^{\prime}-$ pentyl-5-(thiophene-2carbonyl)hexahydrospiro[furo[2,3-c]pyrrole-2,3'-piperidine]-6carboxamide (30). General Method I was applied to methyl $\left(2 S^{*}, 3 a R^{\star}, 6 R^{\star}, 6 a R^{\star}\right)$-6a-ethyl-1'-pentyl-5-(thiophene-2carbonyl)hexahydrospiro[furo[2,3-c]pyrrole-2,3'-piperidine]-6carboxylate $(24)(158 \mathrm{mg}, 0.352 \mathrm{mmol})$. The carboxylic acid $(20$ $\mathrm{mg}, 0.046 \mathrm{mmol})$ and $\mathrm{NH}_{4} \mathrm{Cl}(7.4 \mathrm{mg}, 0.14 \mathrm{mmol})$ were used following General Method J. The mixture was purified using preparative HPLC to provide the title compound as a white solid (12 mg, 59\%). ${ }^{1} \mathrm{H}$ NMR (400 MHz, $\left.\mathrm{CDCl}_{3}\right) \delta 7.52$ (d, J = $3.4 \mathrm{~Hz}$, $1 \mathrm{H}), 7.49(\mathrm{~d}, \mathrm{~J}=4.9 \mathrm{~Hz}, 1 \mathrm{H}), 7.12-7.03(\mathrm{~m}, 1 \mathrm{H}), 6.97(\mathrm{~s}, 1 \mathrm{H})$, $5.78(\mathrm{~s}, 1 \mathrm{H}), 4.98(\mathrm{~s}, 1 \mathrm{H}), 4.42-4.21(\mathrm{~m}, 1 \mathrm{H}), 3.87(\mathrm{dd}, \mathrm{J}=10.7$, $2.9 \mathrm{~Hz}, 1 \mathrm{H}), 2.97(\mathrm{~s}, 1 \mathrm{H}), 2.59-2.27(\mathrm{~m}, 6 \mathrm{H}), 2.17-1.99(\mathrm{~m}$, $2 \mathrm{H}), 1.99-1.76(\mathrm{~m}, 2 \mathrm{H}), 1.73-1.49(\mathrm{~m}, 4 \mathrm{H}), 1.40-1.07(\mathrm{~m}, 6 \mathrm{H})$, $1.01(\mathrm{t}, \mathrm{J}=7.3 \mathrm{~Hz}, 3 \mathrm{H}), 0.82(\mathrm{t}, \mathrm{J}=6.9 \mathrm{~Hz}, 3 \mathrm{H}) ;{ }^{13} \mathrm{C}$ NMR $(101$ $\left.\mathrm{MHz}, \mathrm{CDCl}_{3}\right) \delta 171.7,162.40,138.1,130.7,130.6,127.5,93.7$, 82.5, 70.6, 63.0, 58.6, 55.44, 53.3, 46.5, 41.8, 37.4, 29.7, 27.0, 25.5, 22.6, 22.5, 14.2, 9.0. HRMS (ESI) calcd for $\mathrm{C}_{23} \mathrm{H}_{36} \mathrm{~N}_{3} \mathrm{O}_{3} \mathrm{~S}$ $\left[\mathrm{M}+\mathrm{H}^{+}\right]$434.2472, found 434.2485.

$\left(2 S^{*}, 3 a R^{*}, 6 R^{*}, 6 a R^{*}\right)-6 a-E$ thyl-N,N-dimethyl-1'-pentyl-5(thiophene-2-carbonyl)hexahydrospiro[furo[2,3-c]pyrrole-2,3'piperidine]-6-carboxamide (31). General Method I was applied to methyl $\left(2 S^{*}, 3 a R^{*}, 6 R^{*}, 6 a R^{*}\right)$-6a-ethyl-1'-pentyl-5-(thiophene-2carbonyl)hexahydrospiro[furo[2,3-c]pyrrole-2,3'-piperidine]-6carboxylate $(24)(158 \mathrm{mg}, 0.352 \mathrm{mmol})$. The carboxylic acid $(20$ $\mathrm{mg}, 0.046 \mathrm{mmol}$ ) and dimethylamine hydrochloride $(11 \mathrm{mg}, 0.14$ $\mathrm{mmol}$ ) were used following General Method J. The mixture was purified using preparative HPLC to provide the title compound as a white solid (12 mg, $56 \%) .{ }^{1} \mathrm{H}$ NMR $\left(400 \mathrm{MHz}, \mathrm{CDCl}_{3}\right) \delta 7.50(\mathrm{dd}$, $J=3.7,0.7 \mathrm{~Hz}, 1 \mathrm{H}), 7.43(\mathrm{~d}, J=5.0 \mathrm{~Hz}, 1 \mathrm{H}), 7.02$ (dd, $J=4.9$, $3.9 \mathrm{~Hz}, 1 \mathrm{H}), 5.30(\mathrm{~s}, 1 \mathrm{H}), 4.34(\mathrm{dd}, \mathrm{J}=10.4,9.1 \mathrm{~Hz}, 1 \mathrm{H}), 3.90$ (dd, 
$J=10.7,3.2 \mathrm{~Hz}, 1 \mathrm{H}), 3.26(\mathrm{~s}, 3 \mathrm{H}), 2.99(\mathrm{dd}, \mathrm{J}=9.9,6.9 \mathrm{~Hz}, 1 \mathrm{H})$, $2.90(\mathrm{~s}, 3 \mathrm{H}), 2.87-2.82(\mathrm{~m}, 1 \mathrm{H}), 2.77-2.61(\mathrm{~m}, 1 \mathrm{H}), 2.53-2.40$ $(\mathrm{m}, 2 \mathrm{H}), 2.37-2.22(\mathrm{~m}, 2 \mathrm{H}), 1.98(\mathrm{dd}, \mathrm{J}=13.6,8.0 \mathrm{~Hz}, 1 \mathrm{H}), 1.85$ $-1.74(\mathrm{~m}, 1 \mathrm{H}), 1.69-1.50(\mathrm{~m}, 6 \mathrm{H}), 1.33-1.24(\mathrm{~m}, 2 \mathrm{H}), 1.16-$ $1.02(\mathrm{~m}, 4 \mathrm{H}), 0.90(\mathrm{t}, \mathrm{J}=7.4 \mathrm{~Hz}, 3 \mathrm{H}), 0.75(\mathrm{t}, \mathrm{Jz}=6.8 \mathrm{~Hz}, 3 \mathrm{H})$; ${ }^{13} \mathrm{C}$ NMR $\left(101 \mathrm{MHz}, \mathrm{CDCl}_{3}\right) \delta 170.3,162.0,138.2,130.8,130.6$, 127.6, 94.9, 82.2, 67.3, 61.3, 57.9, 55.7, 53.0, 47.4, 41.8, 38.1, $37.5,35.9,29.4,27.5,24.8,22.3,21.9,14.1,8.9$. HRMS (ESI) calcd for $\mathrm{C}_{25} \mathrm{H}_{40} \mathrm{~N}_{3} \mathrm{O}_{3} \mathrm{~S}\left[\mathrm{M}+\mathrm{H}^{+}\right]$462.2785, found 462.2837 .

\section{$\left(2 S^{*}, 3 a R^{*}, 6 R^{*}, 6 a R^{*}\right)-6 a-E$ thyl-N-(2-methoxyethyl)-1'-pentyl-5-} (thiophene-2-carbonyl)hexahydrospiro[furo[2,3-c]pyrrole-2,3'piperidine]-6-carboxamide (32). General Method I was applied to methyl $\left(2 S^{*}, 3 a R^{*}, 6 R^{*}, 6 a R^{*}\right)$-6a-ethyl-1'-pentyl-5-(thiophene-2carbonyl)hexahydrospiro[furo[2,3-c]pyrrole-2,3'-piperidine]-6carboxylate (24) $(158 \mathrm{mg}, 0.352 \mathrm{mmol})$. The carboxylic acid (20 $\mathrm{mg}, 0.046 \mathrm{mmol})$ and 2-methoxyethylamine $(8 \mu \mathrm{L}, 0.092)$ was used following General Method J. The mixture was purified using preparative HPLC to provide the title compound as a white solid (9 mg, 41\%). ${ }^{1} \mathrm{H}$ NMR (400 MHz, $\left.\mathrm{CDCl}_{3}\right) \delta 8.49(\mathrm{~s}, 1 \mathrm{H}), 7.54$ $(\mathrm{d}, \mathrm{J}=3.3 \mathrm{~Hz}, 1 \mathrm{H}), 7.49(\mathrm{~d}, \mathrm{~J}=4.8 \mathrm{~Hz}, 1 \mathrm{H}), 7.12-7.01(\mathrm{~m}, 1 \mathrm{H})$, $4.91(\mathrm{~s}, 1 \mathrm{H}), 4.32(\mathrm{t}, \mathrm{J}=9.6 \mathrm{~Hz}, 1 \mathrm{H}), 4.15-3.83(\mathrm{~m}, 1 \mathrm{H}), 3.59-$ $3.33(\mathrm{~m}, 4 \mathrm{H}), 3.30(\mathrm{~s}, 3 \mathrm{H}), 2.98(\mathrm{~s}, 1 \mathrm{H}), 2.83-2.35(\mathrm{~m}, 6 \mathrm{H}), 2.25$ $-1.78(\mathrm{~m}, 4 \mathrm{H}), 1.77-1.30(\mathrm{~m}, 6 \mathrm{H}), 1.28-1.09(\mathrm{~m}, 4 \mathrm{H}), 1.00(\mathrm{t}$, $\mathrm{J}=7.2 \mathrm{~Hz}, 3 \mathrm{H}), 0.83(\mathrm{t}, \mathrm{J}=6.8 \mathrm{~Hz}, 3 \mathrm{H}) .{ }^{13} \mathrm{C}$ NMR $(101 \mathrm{MHz}$, $\left.\mathrm{CDCl}_{3}\right) \delta 169.6,168.2,138.2,130.7,130.5,127.5,94.3,82.0$, $71.1,71.0,61.7,58.8,58.1,55.5,52.9,46.7,42.0,39.3,37.2$, $29.5,27.1,24.8,22.4,21.9,14.2,8.9$. HRMS (ESI) calcd for $\mathrm{C}_{26} \mathrm{H}_{42} \mathrm{~N}_{3} \mathrm{O}_{4} \mathrm{~S}\left[\mathrm{M}+\mathrm{H}^{+}\right]$492.2891, found 492.2944.

$\left(2 S^{\star}, 3 a R^{*}, 6 R^{\star}, 6 a R^{*}\right)-N$-Cyclopentyl-6a-ethyl-5-(pyridin-2ylmethyl)-6-(pyrrolidine-1-carbonyl)hexahydrospiro[furo[2,3c]pyrrole-2,3'-piperidine]-1'-carboxamide (33). General Method F was applied to tert-butyl $\left(2 \mathrm{~S}^{*}, 3 \mathrm{a} R^{*}, 6 R^{*}, 6 \mathrm{a} R^{*}\right)$-6a-ethyl-5-(pyridin2-ylmethyl)-6-(pyrrolidine-1-carbonyl)hexahydrospiro[furo[2,3c]pyrrole-2,3'-piperidine]-1'-carboxylate (26) (85 mg, $0.17 \mathrm{mmol}$ ). Following this reaction, General Method $\mathbf{G}$ was applied to TFA amine salt ( $37 \mathrm{mg}, 0.059 \mathrm{mmol})$ and cyclopentyl isocyanate $(8 \mu \mathrm{L}$, $0.071 \mathrm{mmol}$ ) to provide the desired product as a white solid (19 $\mathrm{mg}, 61 \%)$ from purification using the preparative HPLC. ${ }^{1} \mathrm{H}$ NMR $\left(400 \mathrm{MHz}, \mathrm{CDCl}_{3}\right) \delta 8.56(\mathrm{dd}, \mathrm{J}=5.3,1.7 \mathrm{~Hz}, 1 \mathrm{H}), 7.64(\mathrm{td}, \mathrm{J}=$ 7.7, $1.7 \mathrm{~Hz}, 1 \mathrm{H}), 7.21(\mathrm{~d}, J=3.9 \mathrm{~Hz}, 1 \mathrm{H}), 7.20(\mathrm{~d}, J=3.8 \mathrm{~Hz}, 1 \mathrm{H})$ 6.39 (br. s, $1 \mathrm{H}), 4.26-4.07(\mathrm{~m}, 2 \mathrm{H}), 4.05-3.89(\mathrm{~m}, 2 \mathrm{H}), 3.83-$ $3.72(\mathrm{~m}, 3 \mathrm{H}), 3.58-3.47(\mathrm{~m}, 1 \mathrm{H}), 3.48-3.38(\mathrm{~m}, 1 \mathrm{H}), 3.38-$ $3.30(\mathrm{~m}, 1 \mathrm{H}), 3.18(\mathrm{~d}, \mathrm{~J}=12.9 \mathrm{~Hz}, 1 \mathrm{H}), 3.12-3.00(\mathrm{~m}, 1 \mathrm{H}), 2.97$ $-2.85(\mathrm{~m}, 2 \mathrm{H}), 2.70(\mathrm{dd}, \mathrm{J}=9.8,8.0 \mathrm{~Hz}, 1 \mathrm{H}), 2.00-1.88(\mathrm{~m}, 3 \mathrm{H})$, $1.87-1.75(\mathrm{~m}, 5 \mathrm{H}), 1.76-1.67(\mathrm{~m}, 1 \mathrm{H}), 1.66-1.57(\mathrm{~m}, 3 \mathrm{H})$, $1.55-1.38(\mathrm{~m}, 6 \mathrm{H}), 1.37-1.25(\mathrm{~m}, 2 \mathrm{H}), 0.86(\mathrm{t}, \mathrm{J}=7.2 \mathrm{~Hz}, 3 \mathrm{H})$; ${ }^{13} \mathrm{C}$ NMR $\left(101 \mathrm{MHz}, \mathrm{CDCl}_{3}\right) \delta 169.6,163.3,157.9,149.3,136.9$, $123.4,122.8,97.9,83.8,73.2,61.3,56.3,53.1,52.9,47.0,46.0$, 45.6, 43.2, 38.5, 33.4, 33.2, 28.4, 26.3, 24.2, 23.9, 23.8, 23.7 (2C), 8.7. HRMS (ESI) calcd for $\mathrm{C}_{29} \mathrm{H}_{44} \mathrm{~N}_{5} \mathrm{O}_{3}\left[\mathrm{M}+\mathrm{H}^{+}\right]$510.3439, found 510.3493 .

1-((2S*,3aR*,6R*,6aR*)-6a-Ethyl-5-(pyridin-2-ylmethyl)-6(pyrrolidine-1-carbonyl)hexahydrospiro[furo[2,3-c]pyrrole-2,3'piperidin]-1'-yl)ethan-1-one (34). General Method $\mathbf{F}$ was applied to tert-butyl $\left(2 S^{*}, 3 a R^{*}, 6 R^{*}, 6 a R^{*}\right)-6 a-e t h y l-5$-(pyridin-2-ylmethyl)6-(pyrrolidine-1-carbonyl)hexahydrospiro[furo[2,3-c]pyrrole-2,3'piperidine]-1'-carboxylate (26) $(85 \mathrm{mg}, 0.17 \mathrm{mmol})$. Following this reaction, General Method J was applied to TFA amine salt (24 $\mathrm{mg}, 0.060 \mathrm{mmol})$ and acetic acid $(4.5 \mu \mathrm{L}, 0.078 \mathrm{mmol})$ to give after preparative HPLC the title compound as a white foam $(14 \mathrm{mg}$, $54 \%)$. ${ }^{1} \mathrm{H}$ NMR $\left(400 \mathrm{MHz}, \mathrm{CD}_{3} \mathrm{OD}\right) \delta 8.51-8.44(\mathrm{~m}, 1 \mathrm{H}), 7.89-$ $7.81(\mathrm{~m}, 1 \mathrm{H}), 7.75-7.66(\mathrm{~m}, 1 \mathrm{H}), 7.36-7.32(\mathrm{~m}, 1 \mathrm{H}), 4.13-$ $3.99(\mathrm{~m}, 2 \mathrm{H}), 3.80-3.69(\mathrm{~m}, 1 \mathrm{H}), 3.64-3.34(\mathrm{~m}, 7 \mathrm{H}), 3.33-$ $3.26(\mathrm{~m}, 3 \mathrm{H}), 2.85(\mathrm{dd}, \mathrm{J}=9.6,7.8 \mathrm{~Hz}, 1 \mathrm{H}), 2.78-2.65(\mathrm{~m}, 1 \mathrm{H})$, $2.12(\mathrm{~s}, 2.4 \mathrm{H}$, major rotamer), $1.99-1.68(\mathrm{~m}, 8.6 \mathrm{H}), 1.70-1.55$ $(\mathrm{m}, 2 \mathrm{H}), 1.55-1.45(\mathrm{~m}, 1 \mathrm{H}), 0.98-0.81(\mathrm{~m}, 3 \mathrm{H}) ;{ }^{13} \mathrm{C}$ NMR $(101$ $\left.\mathrm{MHz}, \mathrm{CD}_{3} \mathrm{OD}\right) \delta 171.5,170.0,159.0,149.3,138.9,126.0,125.4$, 98.2 , 85.6, 77.1, 61.4, 59.6, 51.9, 47.5, 47.1, 47.0, 40.9, 38.6, $30.3,27.1,27.0,25.0,24.6,21.3,8.4$ (major rotamers). HRMS (ESI) calcd for $\mathrm{C}_{25} \mathrm{H}_{37} \mathrm{~N}_{4} \mathrm{O}_{3}\left[\mathrm{M}+\mathrm{H}^{+}\right]$441.2860, found 441.2895.

Tert-butyl $\left(2 S^{*}, 3 a R^{\star}, 6 R^{\star}, 6 a R^{\star}\right)$-6a-Ethyl-5-(isopropylcarbamoyl)6-(pyrrolidine-1-carbonyl)hexahydrospiro[furo[2,3-c]pyrrole-2,3'piperidine]-1'-carboxylate (35). General Method B was applied to tert-butyl $\quad\left(2 R^{*}, 3 a R^{*}, 6 R^{*}, 6 a R^{*}\right)$-5-benzyl-6-(pyrrolidine-1carbonyl)-6a-vinyl-3,3a, 4,5,6,6a-hexahydro-2'H-spiro[furo[2,3c]pyrrole-2,3'-pyridine]-1'(6'H)-carboxylate (22) (1.32g, 2.68 $\mathrm{mmol})$. Following this reaction, General Method $\mathbf{G}$ was applied to amine (567 mg, $1.39 \mathrm{mmol})$ and isopropyl isocyanate $(145 \mu \mathrm{L}$, $1.47 \mathrm{mmol}$ ). The crude mixture was purified directly using flash column chromatography (EtOAc: $\mathrm{MeOH} 9: 1, R_{\mathrm{f}}=0.2$ ) to give the title compound as a white solid $(685 \mathrm{mg},>95 \%) .{ }^{1} \mathrm{H}$ NMR $(400$ $\left.\mathrm{MHz}, \mathrm{CDCl}_{3}\right) \delta 4.93(\mathrm{~s}, 1 \mathrm{H}), 4.75(\mathrm{~s}, 1 \mathrm{H}), 4.18-3.72(\mathrm{~m}, 5 \mathrm{H})$, $3.72-3.45(\mathrm{~m}, 3 \mathrm{H}), 3.41-3.26(\mathrm{~m}, 1 \mathrm{H}), 3.11-2.43(\mathrm{~m}, 3 \mathrm{H})$, $2.13-1.52(\mathrm{~m}, 12 \mathrm{H}), 1.44(\mathrm{~s}, 9 \mathrm{H}), 1.20-1.03(\mathrm{~m}, 6 \mathrm{H}), 0.90(\mathrm{t}, \mathrm{J}$ $=7.3 \mathrm{~Hz}, 3 \mathrm{H}) ;{ }^{13} \mathrm{C}$ NMR $\left(101 \mathrm{MHz}, \mathrm{CDCl}_{3}\right) \delta 170.7,156.6,154.5$, $96.5,82.6,79.5,68.4,53.8,51.8,47.2,46.5,45.7,44.3,42.5$, $40.7,38.9,28.9,28.5,28.1,26.3$ (3C), 24.5, 24.4, 23.5, 8.9. HRMS (ESI) calcd for $\mathrm{C}_{26} \mathrm{H}_{45} \mathrm{~N}_{4} \mathrm{O}_{5}\left[\mathrm{M}+\mathrm{H}^{+}\right] 493.3384$, found 493.3409.

$\left(2 S^{*}, 3 a R^{*}, 6 R^{*}, 6 a R^{*}\right)-1$ '-Benzyl-6a-ethyl-N-isopropyl-6-

(pyrrolidine-1-carbonyl)tetrahydrospiro[furo[2,3-c]pyrrole-2,3'piperidine]-5(3H)-carboxamide (36). General Method $\mathbf{F}$ was applied to tert-butyl $\left(2 S^{\star}, 3 a R^{\star}, 6 R^{\star}, 6 a R^{\star}\right)$-6a-ethyl-5(isopropylcarbamoyl)-6-(pyrrolidine-1-

carbonyl)hexahydrospiro[furo[2,3-c]pyrrole-2,3'-piperidine]-1'carboxylate (35) $(87 \mathrm{mg}, 0.18 \mathrm{mmol})$. General Method E was applied to resulting amine $(23 \mathrm{mg}, 0.045 \mathrm{mmol})$ and benzaldehyde $(8.8 \mu \mathrm{L}, 0.086 \mathrm{mmol})$. Mixture was purified by preparative HPLC to give a white solid (14 mg, 63\%). ${ }^{1} \mathrm{H}$ NMR $\left(400 \mathrm{MHz}, \mathrm{CDCl}_{3}\right) \delta 7.41-7.37(\mathrm{~m}, 3 \mathrm{H}), 7.35-7.30(\mathrm{~m}, 2 \mathrm{H}), 4.87$ (s, $1 \mathrm{H}), 4.06-3.83(\mathrm{~m}, 5 \mathrm{H}), 3.65-3.44(\mathrm{~m}, 2 \mathrm{H}), 3.44-3.30(\mathrm{~m}$, $1 \mathrm{H}), 3.27(\mathrm{~d}, \mathrm{~J}=8.7 \mathrm{~Hz}, 1 \mathrm{H}), 2.95-2.74(\mathrm{~m}, 3 \mathrm{H}), 2.45-2.19(\mathrm{~m}$, $2 \mathrm{H}), 2.07-1.76(\mathrm{~m}, 6 \mathrm{H}), 1.77-1.49(\mathrm{~m}, 6 \mathrm{H}), 1.13(\mathrm{t}, \mathrm{J}=6.4 \mathrm{~Hz}$, $6 \mathrm{H}), 0.89(\mathrm{t}, J=7.4 \mathrm{~Hz}, 3 \mathrm{H}) ;{ }^{13} \mathrm{C}$ NMR $\left(101 \mathrm{MHz}, \mathrm{CDCl}_{3}\right) \delta 170.1$, 156.7, 131.0, 129.3 (2C), 129.1 (2C), 97.5, 81.9, 68.0, 61.3, 60.1, 53.8, 51.0, 47.3, 46.6, 45.8, 42.6, 42.4, 36.9, 28.0, 26.3, 24.3, 23.6, 23.2 (2C), 21.6, 8.9. HRMS (ESI) calcd for $\mathrm{C}_{28} \mathrm{H}_{43} \mathrm{~N}_{4} \mathrm{O}_{3}$ $\left[\mathrm{M}+\mathrm{H}^{+}\right]$483.3330, found 483.3384 .

$\left(2 S^{*}, 3 a R^{*}, 6 R^{*}, 6 a R^{*}\right)-6 a-E$ thyl-N-isopropyl-6-(pyrrolidine-1carbonyl)-1'-tosyltetrahydrospiro[furo[2,3-c]pyrrole-2,3'piperidine]-5(3H)-carboxamide (37). General Method F was applied to tert-butyl $\left(2 S^{\star}, 3 a R^{*}, 6 R^{*}, 6 a R^{*}\right)-6 a-e t h y l-5$ (isopropylcarbamoyl)-6-(pyrrolidine-1-

carbonyl)hexahydrospiro[furo[2,3-c]pyrrole-2,3'-piperidine]-1'carboxylate $(35)(87 \mathrm{mg}, 0.18 \mathrm{mmol})$. General Method K was applied to the resulting amine $(20 \mathrm{mg}, 0.040 \mathrm{mmol})$ and 4 toluenesulfonyl chloride (12 $\mathrm{mg}, 0.062 \mathrm{mmol})$. Mixture was purified directly by preparative HPLC to give a white solid $(13 \mathrm{mg}$, $57 \%) .{ }^{1} \mathrm{H}$ NMR $\left(400 \mathrm{MHz}, \mathrm{CDCl}_{3}\right) \delta 7.56(\mathrm{~d}, \mathrm{~J}=8.2 \mathrm{~Hz}, 2 \mathrm{H}), 7.29$ $(\mathrm{d}, \mathrm{J}=8.2 \mathrm{~Hz}, 2 \mathrm{H}), 4.71(\mathrm{~s}, 1 \mathrm{H}), 4.51(\mathrm{~d}, \mathrm{~J}=7.6 \mathrm{~Hz}, 1 \mathrm{H}), 4.11(\mathrm{t}$, $\mathrm{J}=9.4 \mathrm{~Hz}, 1 \mathrm{H}), 4.07-3.89(\mathrm{~m}, 2 \mathrm{H}), 3.73(\mathrm{~d}, \mathrm{~J}=11.5 \mathrm{~Hz}, 1 \mathrm{H})$, $3.63(\mathrm{~d}, \mathrm{~J}=11.0 \mathrm{~Hz}, 1 \mathrm{H}), 3.59-3.42(\mathrm{~m}, 3 \mathrm{H}), 3.43-3.29(\mathrm{~m}$, $1 \mathrm{H}), 3.03-2.85(\mathrm{~m}, 1 \mathrm{H}), 2.52(\mathrm{~d}, \mathrm{~J}=13.3 \mathrm{~Hz}, 1 \mathrm{H}), 2.41(\mathrm{~s}, 3 \mathrm{H})$, $2.15-1.96(\mathrm{~m}, 3 \mathrm{H}), 1.94-1.53(\mathrm{~m}, 9 \mathrm{H}), 1.54-1.38(\mathrm{~m}, 1 \mathrm{H})$, $1.22(\mathrm{~d}, \mathrm{~J}=6.4 \mathrm{~Hz}, 3 \mathrm{H}), 1.15(\mathrm{~d}, \mathrm{~J}=6.6 \mathrm{~Hz}, 3 \mathrm{H}), 0.89(\mathrm{t}, \mathrm{J}=7.3$ $\mathrm{Hz}, 3 \mathrm{H}) ;{ }^{13} \mathrm{C}$ NMR $\left(101 \mathrm{MHz}, \mathrm{CDCl}_{3}\right) \delta 170.8,156.6,143.7,133.6$, 
129.9 (2C), $127.4(2 \mathrm{C}), 96.6,82.7,68.8,54.6,54.5,47.2,46.7$, 46.0, 45.7, 42.7, 40.9, 38.0, 28.2, 26.3, 24.4, 23.6 (2C), 23.4, 21.6, 8.9. HRMS (ESI) calcd for $\mathrm{C}_{28} \mathrm{H}_{43} \mathrm{~N}_{4} \mathrm{O}_{5} \mathrm{~S}\left[\mathrm{M}+\mathrm{H}^{+}\right]$547.2949, found 547.2947 .

$\left(2 S^{\star}, 3 a R^{\star}, 6 R^{*}, 6 a R^{*}\right)-6 a-E t h y l-1 '-i s o n i c o t i n o y l-N-i s o p r o p y l-6-$ (pyrrolidine-1-carbonyl)tetrahydrospiro[furo[2,3-c]pyrrole-2,3'piperidine]-5(3H)-carboxamide (38). General Method F was applied to tert-butyl $\left(2 S^{*}, 3 a R^{\star}, 6 R^{\star}, 6 a R^{\star}\right)$-6a-ethyl-5(isopropylcarbamoyl)-6-(pyrrolidine-1-

carbonyl)hexahydrospiro[furo[2,3-c]pyrrole-2,3'-piperidine]-1'carboxylate (35) (87 mg, $0.18 \mathrm{mmol})$. General Method I was then applied to resulting amine $(22 \mathrm{mg}, 0.043 \mathrm{mmol})$ and isonicotinic acid $(7 \mathrm{mg}, 0.056 \mathrm{mmol})$. The crude mixture was purified using preparative HPLC to give the title compound as a white solid (15 $\mathrm{mg}, 69 \%) .{ }^{1} \mathrm{H}$ NMR $\left(400 \mathrm{MHz}, \mathrm{CDCl}_{3}\right) \delta 8.69$ (dd, $J=4.5,1.5 \mathrm{~Hz}$, $2 \mathrm{H}), 7.31(\mathrm{~d}, J=5.1 \mathrm{~Hz}, 1 \mathrm{H}), 7.24(\mathrm{dd}, J=4.5,1.5 \mathrm{~Hz}, 2 \mathrm{H}), 4.83$ $(\mathrm{s}, 1 \mathrm{H}), 4.68(\mathrm{~d}, \mathrm{~J}=7.8 \mathrm{~Hz}, 1 \mathrm{H}), 4.45(\mathrm{~d}, \mathrm{~J}=12.9 \mathrm{~Hz}, 1 \mathrm{H}), 4.11-$ $4.00(\mathrm{~m}, 2 \mathrm{H}), 3.98-3.83(\mathrm{~m}, 1 \mathrm{H}), 3.71(\mathrm{dd}, \mathrm{J}=9.4,3.1 \mathrm{~Hz}, 1 \mathrm{H})$, $3.57-3.48(\mathrm{~m}, 2 \mathrm{H}), 3.45(\mathrm{~d}, \mathrm{~J}=13.5 \mathrm{~Hz}, 1 \mathrm{H}), 3.40-3.32(\mathrm{~m}$, $1 \mathrm{H}), 3.07-2.93(\mathrm{~m}, 2 \mathrm{H}), 2.81(\mathrm{~d}, \mathrm{~J}=13.1 \mathrm{~Hz}, 1 \mathrm{H}), 2.12(\mathrm{~d}, \mathrm{~J}=$ $13.1 \mathrm{~Hz}, 1 \mathrm{H}), 2.05-1.77(\mathrm{~m}, 7 \mathrm{H}), 1.74-1.58(\mathrm{~m}, 2 \mathrm{H}), 1.44-$ $1.34(\mathrm{~m}, 1 \mathrm{H}), 1.12(\mathrm{~d}, \mathrm{~J}=3.6 \mathrm{~Hz}, 3 \mathrm{H}), 1.11(\mathrm{~d}, \mathrm{~J}=3.8 \mathrm{~Hz}, 3 \mathrm{H})$, $0.93(\mathrm{t}, \mathrm{J}=7.4 \mathrm{~Hz}, 3 \mathrm{H}) ;{ }^{13} \mathrm{C}$ NMR $\left(101 \mathrm{MHz}, \mathrm{CDCl}_{3}\right) \delta 170.5$, 163.0, 156.5, 150.4 (2C), 144.0, 121.0 (2C), 96.8, 82.5, 68.3, $53.5,50.8,47.6,47.2,46.8,45.8,42.6,40.7,39.0,28.2,26.3$, $24.9,24.4,23.6,23.4,8.9$. HRMS (ESI) calcd for $\mathrm{C}_{27} \mathrm{H}_{40} \mathrm{~N}_{5} \mathrm{O}_{4}$ $\left[\mathrm{M}+\mathrm{H}^{+}\right]$498.3075, found 498.3072 .

$\left(2 S^{*}, 3 a R^{*}, 6 R^{*}, 6 a R^{*}\right)-1^{\prime}-$ Benzoyl-6a-ethyl-N5-hexyl-N6isopropyltetrahydrospiro[furo[2,3-c]pyrrole-2,3'-piperidine]5,6(3H)-dicarboxamide (39). General Method $\mathbf{G}$ was applied to $\left(2 S^{*}, 3 a R^{*}, 6 R^{*}, 6 a R^{*}\right)-1^{\prime}$-benzoyl-6a-ethyl- $N$ -

isopropylhexahydrospiro[furo[2,3-c]pyrrole-2,3'-piperidine]-6carboxamide (S10) $(20 \mathrm{mg}, 0.050 \mathrm{mmol})$ and hexyl isocyanate $(8.8 \mu \mathrm{L}, 0.060 \mathrm{mmol})$ to give after preparative HPLC the title compound as a white foam (18 mg, 69\%). ${ }^{1} \mathrm{H}$ NMR $(400 \mathrm{MHz}$, $\left.\mathrm{CDCl}_{3}\right) \delta 7.46-7.38(\mathrm{~m}, 3 \mathrm{H}), 7.38-7.32(\mathrm{~m}, 2 \mathrm{H}), 6.49(\mathrm{~d}, \mathrm{~J}=7.4$ $\mathrm{Hz}, 1 \mathrm{H}), 4.82(\mathrm{t}, \mathrm{J}=5.1 \mathrm{~Hz}, 1 \mathrm{H}), 4.38(\mathrm{~s}, 1 \mathrm{H}), 4.09-3.94(\mathrm{~m}, 2 \mathrm{H})$, $3.89-3.72(\mathrm{~m}, 1 \mathrm{H}), 3.52-3.38(\mathrm{~m}, 1 \mathrm{H}), 3.38-3.06(\mathrm{~m}, 4 \mathrm{H})$, $2.97-2.74(\mathrm{~m}, 1 \mathrm{H}), 2.10(\mathrm{dd}, \mathrm{J}=13.0,3.0 \mathrm{~Hz}, 1 \mathrm{H}), 2.01-1.56$ $(\mathrm{m}, 6 \mathrm{H}), 1.56-1.37(\mathrm{~m}, 3 \mathrm{H}), 1.31-1.20(\mathrm{~m}, 6 \mathrm{H}), 1.13(\mathrm{~d}, \mathrm{~J}=6.6$ $\mathrm{Hz}, 3 \mathrm{H}), 1.10(\mathrm{~d}, \mathrm{~J}=6.5 \mathrm{~Hz}, 3 \mathrm{H}), 0.96(\mathrm{t}, \mathrm{J}=7.4 \mathrm{~Hz}, 3 \mathrm{H}), 0.90-$ $0.80(\mathrm{~m}, 3 \mathrm{H}) .{ }^{13} \mathrm{C}$ NMR $\left(101 \mathrm{MHz}, \mathrm{CDCl}_{3}\right) \delta 170.6,169.9,157.5$, 136.3, 129.7, 128.7 (2C), $126.6(2 \mathrm{C}), 95.7,82.3,70.0,51.7,47.9$, 45.4, 41.4, 41.2, s41.0, 40.4, 38.8, 31.7 (2C), 30.3, 27.2, 26.6, $24.4,22.7,22.5,14.2,8.8$. HRMS (ESI) calcd for $\mathrm{C}_{30} \mathrm{H}_{47} \mathrm{~N}_{4} \mathrm{O}_{4}$ $\left[\mathrm{M}+\mathrm{H}^{+}\right]$527.3592, found 527.3612.

$\left(2 S^{*}, 3 a R^{*}, 6 R^{*}, 6 a R^{*}\right)-1^{\prime}-$ Benzoyl-5-(cyclohexylmethyl)-6a-ethyl$\mathrm{N}$-isopropylhexahydrospiro[furo[2,3-c]pyrrole-2,3'-piperidine]-6carboxamide (40). General Method E was applied to $\left(2 S^{\star}, 3 a R^{*}, 6 R^{*}, 6 a R^{\star}\right)-1$ '-benzoyl-6a-ethyl- $N$ -

isopropylhexahydrospiro[furo[2,3-c]pyrrole-2,3'-piperidine]-6carboxamide (S10) $(17 \mathrm{mg}, 0.043 \mathrm{mmol}$ ) and cyclohexane carbaldehyde $(7.7 \mu \mathrm{L}, 0.064 \mathrm{mmol})$ gave after preparative HPLC the title compound as a white foam $(15 \mathrm{mg}, 72 \%) .{ }^{1} \mathrm{H}$ NMR (400 $\left.\mathrm{MHz}, \mathrm{CDCl}_{3}\right) \delta 7.47-7.31(\mathrm{~m}, 5 \mathrm{H}), 6.76(\mathrm{~s}, 0.5 \mathrm{H}), 6.49(\mathrm{~d}, \mathrm{~J}=$ $7.8 \mathrm{~Hz}, 0.5 \mathrm{H}), 4.34(\mathrm{~d}, \mathrm{~J}=12.4 \mathrm{~Hz}, 1 \mathrm{H}), 4.18-3.98(\mathrm{~m}, 1 \mathrm{H}), 3.80$ $(\mathrm{d}, \mathrm{J}=13.0 \mathrm{~Hz}, 0.5 \mathrm{H}), 3.53-3.42(\mathrm{~m}, 1 \mathrm{H}), 3.41-3.20(\mathrm{~m}, 1.5 \mathrm{H})$, $3.20-2.86(\mathrm{~m}, 2 \mathrm{H}), 2.74-2.56(\mathrm{~m}, 1 \mathrm{H}), 2.45-2.22(\mathrm{~m}, 1.5 \mathrm{H})$, $2.08-1.95(\mathrm{~m}, 1.5 \mathrm{H}), 1.88-1.60(\mathrm{~m}, 10 \mathrm{H}), 1.55-1.42(\mathrm{~m}, 2 \mathrm{H})$, $1.34-1.05(\mathrm{~m}, 11 \mathrm{H}), 0.99-0.58(\mathrm{~m}, 5 \mathrm{H})$ (two rotamers) ${ }^{13} \mathrm{C}$ NMR $\left(101 \mathrm{MHz}, \mathrm{CDCl}_{3}\right) \delta 170.5,169.7,136.3,129.8,128.7,128.6$, $127.8,126.8,96.2,84.7,81.4,62.5,58.3,57.3,51.9,47.8,46.1$ $42.3,40.6,38.5,38.2,36.1,32.0,31.6,31.2,28.5,26.8,26.2$,
22.9, 8.2 (major rotamers). HRMS (ESI) calcd for $\mathrm{C}_{30} \mathrm{H}_{46} \mathrm{~N}_{3} \mathrm{O}_{3}$ $\left[\mathrm{M}+\mathrm{H}^{+}\right]$496.3534, found 496.3594 .

$\left(2 R^{*}, 3 a R^{*}, 6 R^{*}, 6 a R^{\star}\right)-5-B e n z y l-N$-isopropyl-1'-(methylsulfonyl)6a-vinyl-1',3,3a,4,5,6,6a, 6'-octahydro-2'H-spiro[furo[2,3c]pyrrole-2,3'-pyridine]-6-carboxamide (41). General Method F was applied to tert-butyl $\left(2 R^{\star}, 3 a R^{*}, 6 R^{*}, 6 a R^{*}\right)-5$-benzyl-6(isopropylcarbamoyl)-6a-vinyl-3,3a,4,5,6,6a-hexahydro-2' $\mathrm{H}$ spiro[furo[2,3-c]pyrrole-2,3'-pyridine]-1'(6'H)-carboxylate $(1.439 \mathrm{~g}, 2.99 \mathrm{mmol})$. General Method $\mathrm{K}$ was applied to amine (570 mg, $1.49 \mathrm{mmol})$ and methanesulfonyl chloride $(210 \mu \mathrm{L}, 1.79$ $\mathrm{mmol}$ ) purified using flash column chromatography (EtOAc:heptane 3:2, $R_{\mathrm{f}}=0.1$ ) to give the title compound as a white solid (485 mg, 71\%). ${ }^{1} \mathrm{H}$ NMR $\left(400 \mathrm{MHz}, \mathrm{CDCl}_{3}\right) \delta 7.37-$ $7.30(\mathrm{~m}, 2 \mathrm{H}), 7.28(\mathrm{~d}, \mathrm{~J}=7.1 \mathrm{~Hz}, 3 \mathrm{H}), 6.57(\mathrm{~d}, \mathrm{~J}=8.2 \mathrm{~Hz}, 1 \mathrm{H})$, $5.96(\mathrm{dd}, J=17.3,10.7 \mathrm{~Hz}, 1 \mathrm{H}), 5.79(\mathrm{dt}, J=10.2,2.0 \mathrm{~Hz}, 1 \mathrm{H})$, $5.69(\mathrm{dt}, J=10.2,3.1 \mathrm{~Hz}, 1 \mathrm{H}), 5.26(\mathrm{dd}, J=17.3,1.3 \mathrm{~Hz}, 1 \mathrm{H})$, $5.10(\mathrm{dd}, J=10.7,1.3 \mathrm{~Hz}, 1 \mathrm{H}), 4.15-3.98(\mathrm{~m}, 1 \mathrm{H}), 3.89(\mathrm{~d}, J=$ $13.0 \mathrm{~Hz}, 1 \mathrm{H}), 3.87-3.79(\mathrm{~m}, 1 \mathrm{H}), 3.66(\mathrm{dt}, \mathrm{J}=17.4,2.5 \mathrm{~Hz}, 1 \mathrm{H})$, $3.55(\mathrm{~d}, \mathrm{~J}=12.5 \mathrm{~Hz}, 1 \mathrm{H}), 3.46(\mathrm{~d}, \mathrm{~J}=2.0 \mathrm{~Hz}, 1 \mathrm{H}), 3.43(\mathrm{~d}, \mathrm{~J}=$ $2.7 \mathrm{~Hz}, 1 \mathrm{H}), 3.35(\mathrm{~s}, 1 \mathrm{H}), 3.29(\mathrm{t}, \mathrm{J}=8.6 \mathrm{~Hz}, 1 \mathrm{H}), 2.92(\mathrm{~s}, 3 \mathrm{H})$, $2.88-2.79(\mathrm{~m}, 1 \mathrm{H}), 2.50(\mathrm{t}, \mathrm{J}=9.0 \mathrm{~Hz}, 1 \mathrm{H}), 2.05-1.90(\mathrm{~m}, 2 \mathrm{H})$, $1.13(\mathrm{~d}, \mathrm{~J}=4.0 \mathrm{~Hz}, 3 \mathrm{H}), 1.11(\mathrm{~d}, \mathrm{~J}=3.9 \mathrm{~Hz}, 3 \mathrm{H}) ;{ }^{13} \mathrm{C}$ NMR $(101$ $\left.\mathrm{MHz}, \mathrm{CDCl}_{3}\right) \delta 168.9,138.6,137.8,133.4,129.0,128.7(2 \mathrm{C})$, 127.6 (2C), 123.1, 114.0, 96.6, 81.9, 78.7, 58.5, 58.0, 53.2, 46.9, $44.2,40.7,38.8,36.8,23.1,22.9$. HRMS (ESI) calcd for $\mathrm{C}_{24} \mathrm{H}_{34} \mathrm{~N}_{3} \mathrm{O}_{4} \mathrm{~S}\left[\mathrm{M}+\mathrm{H}^{+}\right]$460.2265, found 460.2298 .

$\left(2 S^{*}, 3 a R^{*}, 6 R^{*}, 6 a R^{*}\right)-6 a-E$ thyl-N-isopropyl-1'-

(methylsulfonyl)hexahydrospiro[furo[2,3-c]pyrrole-2,3'-

piperidine]-6-carboxamide (S11). ( $\left.2 R^{\star}, 3 a R^{*}, 6 R^{*}, 6 a R^{\star}\right)-5$-benzylN-isopropyl-1'-(methylsulfonyl)-6a-vinyl-1',3,3a, 4,5,6,6a,6'octahydro-2'H-spiro[furo[2,3-c]pyrrole-2,3'-pyridine]-6-

carboxamide (41) (365 mg, $0.79 \mathrm{mmol}$ ) was hydrogenated following General Method B to give the title compound as a white solid (298 mg, 95\%). The amine was used directly without further purification.

$\left(2 S^{\star}, 3 a R^{\star}, 6 R^{\star}, 6 a R^{\star}\right)-6 a-E$ thyl-N6-isopropyl-1'-(methylsulfonyl)N5-(o-tolyl)tetrahydrospiro[furo[2,3-c]pyrrole-2,3'-piperidine]$5,6(3 \mathrm{H})$-dicarboxamide (42). General Method $\mathbf{G}$ was applied to $\left(2 S^{*}, 3 a R^{*}, 6 R^{\star}, 6 a R^{*}\right)-6$ a-ethyl- $N$-isopropyl-1'-

(methylsulfonyl)hexahydrospiro[furo[2,3-c]pyrrole-2,3'-

piperidine]-6-carboxamide (S11) (20 mg, $0.054 \mathrm{mmol}$ ) and o-tolyl isocyanate $(6.8 \mu \mathrm{L}, 0.064 \mathrm{mmol})$ and provide after preparative HPLC, the title compound as a white foam (19 mg, 72\%). ${ }^{1} \mathrm{H}$ NMR $\left(400 \mathrm{MHz}, \mathrm{CDCl}_{3}\right) \delta 7.71(\mathrm{~d}, \mathrm{~J}=7.9 \mathrm{~Hz}, 1 \mathrm{H}), 7.13$ (dd, $\mathrm{J}=$ 9.8, 3.3 Hz, 2H), 6.98 (td, J = 7.5, $1.1 \mathrm{~Hz}, 1 \mathrm{H}), 6.33(\mathrm{~s}, 1 \mathrm{H}), 6.23$ $(\mathrm{d}, \mathrm{J}=7.7 \mathrm{~Hz}, 1 \mathrm{H}), 4.42(\mathrm{~s}, 1 \mathrm{H}), 4.10(\mathrm{t}, \mathrm{J}=9.5 \mathrm{~Hz}, 1 \mathrm{H}), 4.02(\mathrm{dq}$, $\mathrm{J}=13.2,6.6 \mathrm{~Hz}, 1 \mathrm{H}), 3.63(\mathrm{dd}, \mathrm{J}=9.6,3.3 \mathrm{~Hz}, 1 \mathrm{H}), 3.59-3.49$ $(\mathrm{m}, 2 \mathrm{H}), 2.98(\mathrm{t}, \mathrm{J}=8.2 \mathrm{~Hz}, 1 \mathrm{H}), 2.87-2.76(\mathrm{~m}, 2 \mathrm{H}), 2.73(\mathrm{~s}, 3 \mathrm{H})$, $2.38(\mathrm{~d}, \mathrm{~J}=13.6 \mathrm{~Hz}, 1 \mathrm{H}), 2.25(\mathrm{~s}, 3 \mathrm{H}), 1.93-1.54(\mathrm{~m}, 7 \mathrm{H}), 1.13$ (d, $J=6.6 \mathrm{~Hz}, 3 \mathrm{H}), 1.11(\mathrm{~d}, J=6.6 \mathrm{~Hz}, 3 \mathrm{H}), 0.98$ (t, $J=7.4 \mathrm{~Hz}$, $3 \mathrm{H}) ;{ }^{13} \mathrm{C}$ NMR $\left(101 \mathrm{MHz}, \mathrm{CDCl}_{3}\right) \delta 169.7,154.6,137.0,130.5$, $129.3,126.6,124.0,122.4,95.9,82.0,70.6,54.5,53.3,46.4$, 45.6, 41.7, 40.5, 38.3, 36.3, 28.0, 23.6, 22.5, 22.5, 17.9, 9.0. HRMS (ESI) calcd for $\mathrm{C}_{25} \mathrm{H}_{39} \mathrm{~N}_{4} \mathrm{O}_{5} \mathrm{~S}\left[\mathrm{M}+\mathrm{H}^{+}\right]$507.2636, found 507.2645 .

$\left(2 S^{\star}, 3 a R^{\star}, 6 R^{\star}, 6 a R^{\star}\right)-6 a-E$ thyl-N-isopropyl-1'-(methylsulfonyl)-5(thiophen-3-ylmethyl)hexahydrospiro[furo[2,3-c]pyrrole-2,3'piperidine]-6-carboxamide (43). General Method E was applied to $\quad\left(2 S^{\star}, 3 a R^{\star}, 6 R^{\star}, 6 a R^{*}\right)$-6a-ethyl- $N$-isopropyl-1'(methylsulfonyl)hexahydrospiro[furo[2,3-c]pyrrole-2,3'-

piperidine]-6-carboxamide (S11) $(20 \mathrm{mg}, 0.053 \mathrm{mmol})$ and 3thiophenecarboxaldehyde $(7.5 \mu \mathrm{L}, 0.080 \mathrm{mmol})$ gave after 
preparative HPLC the title compound as a white foam $(17 \mathrm{mg}$, $68 \%) .{ }^{1} \mathrm{H}$ NMR $\left(400 \mathrm{MHz}, \mathrm{CDCl}_{3}\right) \delta 7.28(\mathrm{dd}, J=4.9,3.0 \mathrm{~Hz}, 1 \mathrm{H})$, $7.13(\mathrm{~d}, J=2.6 \mathrm{~Hz}, 1 \mathrm{H}), 6.99(\mathrm{dd}, J=4.9,1.2 \mathrm{~Hz}, 1 \mathrm{H}), 6.54(\mathrm{~d}, J$ $=8.1 \mathrm{~Hz}, 1 \mathrm{H}), 4.25-4.01(\mathrm{~m}, 1 \mathrm{H}), 3.85(\mathrm{~d}, \mathrm{~J}=13.4 \mathrm{~Hz}, 1 \mathrm{H}), 3.53$ $-3.42(\mathrm{~m}, 2 \mathrm{H}), 3.43-3.35(\mathrm{~m}, 1 \mathrm{H}), 3.35-3.28(\mathrm{~m}, 1 \mathrm{H}), 3.31(\mathrm{~s}$, $1 \mathrm{H}), 3.20(\mathrm{~d}, \mathrm{~J}=12.3 \mathrm{~Hz}, 1 \mathrm{H}), 3.10-2.98(\mathrm{~m}, 1 \mathrm{H}), 2.93(\mathrm{~s}, 3 \mathrm{H})$, $2.58(\mathrm{td}, \mathrm{J}=10.0,1.9 \mathrm{~Hz}, 1 \mathrm{H}), 2.52-2.45(\mathrm{~m}, 1 \mathrm{H}), 1.95(\mathrm{dd}, \mathrm{J}=$ 13.5, $2.0 \mathrm{~Hz}, 1 \mathrm{H}$ ), 1.84 (dddd, $\mathrm{J}=13.9,10.5,6.9,3.7 \mathrm{~Hz}, 1 \mathrm{H}$ ), $1.80-1.62(\mathrm{~m}, 4 \mathrm{H}), 1.62-1.43(\mathrm{~m}, 2 \mathrm{H}), 1.17(\mathrm{~d}, \mathrm{~J}=6.3 \mathrm{~Hz}, 3 \mathrm{H})$, $1.15(\mathrm{~d}, J=6.4 \mathrm{~Hz}, 3 \mathrm{H}), 0.89(\mathrm{t}, \mathrm{J}=7.3 \mathrm{~Hz}, 3 \mathrm{H}) ;{ }^{13} \mathrm{C}$ NMR $(101$ $\left.\mathrm{MHz}, \mathrm{CDCl}_{3}\right) \delta 169.6,138.6,128.2,126.0,123.1,96.9,83.6,79.1$, 59.1, 55.2, 53.2, 45.7, 45.5, 40.8, 39.9, 37.4, 37.0, 28.5, 23.0, 22.94, 22.91, 8.3. HRMS (ESI) calcd for $\mathrm{C}_{22} \mathrm{H}_{36} \mathrm{~N}_{3} \mathrm{O}_{4} \mathrm{~S}_{2}\left[\mathrm{M}+\mathrm{H}^{+}\right]$ 470.2142 , found 470.2181 .

(2S*,3aR* $\left.{ }^{*} R^{*}, 6 a R^{*}\right)-6 a-E$ thyl-5-(1H-indole-5-carbonyl)- $N$ isopropyl-1'-(methylsulfonyl)hexahydrospiro[furo[2,3-c]pyrrole2,3'-piperidine]-6-carboxamide (44). Following General Method J using $\quad\left(2 S^{*}, 3 a R^{\star}, 6 R^{\star}, 6 a R^{\star}\right)$-6a-ethyl- $N$-isopropyl-1'(methylsulfonyl)hexahydrospiro[furo[2,3-c]pyrrole-2,3'-

piperidine]-6-carboxamide (S11v) $(24 \mathrm{mg}, 0.064 \mathrm{mmol})$, indole-5carboxylic acid (14 mg, $0.084 \mathrm{mmol})$ gave after preparative HPLC the title compound as a white foam $(25 \mathrm{mg}, 74 \%) .{ }^{1} \mathrm{H}$ NMR $(400$ $\left.\mathrm{MHz}, \mathrm{CDCl}_{3}\right) \delta 9.07(\mathrm{~s}, 1 \mathrm{H}), 7.90(\mathrm{~s}, 1 \mathrm{H}), 7.46-7.16(\mathrm{~m}, 3 \mathrm{H})$, $6.56(\mathrm{dd}, \mathrm{J}=29.2,11.9 \mathrm{~Hz}, 2 \mathrm{H}), 4.87(\mathrm{~s}, 1 \mathrm{H}), 4.31-4.01(\mathrm{~m}, 2 \mathrm{H})$, $3.53(\mathrm{dd}, \mathrm{J}=11.4,2.6 \mathrm{~Hz}, 1 \mathrm{H}), 3.18(\mathrm{dt}, \mathrm{J}=48.2,9.0 \mathrm{~Hz}, 4 \mathrm{H})$, $2.93-2.87(\mathrm{~m}, 1 \mathrm{H}), 2.83(\mathrm{~s}, 3 \mathrm{H}), 1.99-1.82(\mathrm{~m}, 4 \mathrm{H}), 1.71-1.51$ $(\mathrm{m}, 4 \mathrm{H}), 1.17-1.15(\mathrm{~m}, 6 \mathrm{H}), 1.03(\mathrm{t}, \mathrm{J}=7.3 \mathrm{~Hz}, 3 \mathrm{H}$ ) (major rotamer); ${ }^{13} \mathrm{C}$ NMR $\left(101 \mathrm{MHz}, \mathrm{CDCl}_{3}\right) \delta 171.9,168.7,137.1$, 127.3, 126.9, 125.7, 121.7, 120.9, 111.2, 103.1, 94.9, 81.2, 69.3, $55.7,55.4,46.2,45.2,41.6,40.5,37.8,37.7,26.9,22.7,22.6$, $22.5,8.9$ (major rotamer). HRMS (ESI) calcd for $\mathrm{C}_{26} \mathrm{H}_{37} \mathrm{~N}_{4} \mathrm{O}_{5} \mathrm{~S}$ $\left[\mathrm{M}+\mathrm{H}^{+}\right]$517.2479, found 517.2476.

\section{Acknowledgments}

The research leading to these results has received support from the Innovative Medicines Initiative Joint Undertaking under grant agreement no. 115489, resources of which are composed of financial contribution from the European Union's Seventh Framework Programme (FP7/2007-2013) and EFPIA companies' in-kind contribution. We are grateful to DTU Chemistry for an academic excellence PhD scholarship to NST. We thank Agathe Gueret, Guillaume Ranty, and Laure Carrier for skillful technical assistance.

Keywords: Drug discovery; Heterocycles; Cycloaddition

[1] R. Macarron, M. N. Banks, D. Bojanic, D. J. Burns, D. A. Cirovic, T. Garyantes, D. V. S. Green, R. P. Hertzberg, W. P. Janzen, J. W. Paslay and U. Schopfer, Nature 2011, 10, 188-195.

[2] (a) T. J. Ritchie and S. J. F. Macdonald, Drug Discov. Today, 2009, 14, 1011-1020; (b) F. Lovering, J. Bikker and C. Humblet, J. Med. Chem., 2009, 52, 6752-6756; (c) F. Lovering, MedChemComm 2013, 4, 515519; (d) A. Nadin, C. Hattotuwagama and I. Churcher, Angew. Chemie Int. Ed. 2012, 51, 1114-1122; Angew. Chem. 2012, 124, 1140-1149; (e)
A. Chuprina, O. Lukin, R. Demoiseaux, A. Buzko and A. Shivanyuk, J. Chem. Inf. Model. 2010, 50, 470-479.

[3] (a) C. M. Dobson, Nature 2004, 432, 824-829; (b) R. A. Bauer, J. M. Wurst and D. S. Tan, Curr. Opin. Chem. Biol. 2010, 14, 308-314; (c) S. Dandapani and L. A. Marcaurelle, Nat. Chem. Biol. 2010, 6, 861-863; (d) C. J. O' Connor, H. S. G. Beckmann and D. R. Spring, Chem. Soc. Rev. 2012, 41, 4444-4456; (e) D. H. Drewry and R. Macarron, Curr. Opin. Chem. Biol. 2010, 14, 289-298.

[4] (a) D. R. Spring, Org. Biomol. Chem. 2003, 1, 3867-3870; (b) S. L. Schreiber, Science 2000, 287, 1964-1969; (c) D. S. Tan, Nat. Chem. Biol. 2005, 1, 74-84.

[5] A. Karawajczyk, F. Giordanetto, J. Benningshof, D. Hamza, T. Kalliokoski, K. Pouwer, R. Morgentin, A. Nelson, G. Müller, A. Piechot and D. Tzalis, Drug Discov. Today 2015, 20, 1310-1316.

[6] (a) P. Wu, M. A. Petersen, A. E. Cohrt, R. Petersen, M. H. Clausen and T. E. Nielsen, Eur. J. Org. Chem. 2015, 2015, 2346-2350; (b) P. Wu, M. Å. Petersen, R. Petersen, M. O. Rasmussen, K. Bonnet, T. E. Nielsen and M. H. Clausen, Eur. J. Org. Chem. 2015, 2015, 5633-5639; (c) R. Petersen, A. E. Cohrt, M. Å. Petersen, P. Wu, M. H. Clausen and T. E. Nielsen, Bioorg. Med. Chem. 2015, 23, 2646-2649; (d) M. A. Petersen, M. A. Mortensen, A. E. Cohrt, R. Petersen, P. Wu, N. Fleury-Brégeot, R. Morgentin, C. Lardy, T. E. Nielsen and M. H. Clausen, Bioorg. Med. Chem. 2015, 23, 2695-2698; (e) P. Wu, M. Å. Petersen, R. Petersen, T. Flagstad, R. Guilleux, M. Ohsten, R. Morgentin, T. E. Nielsen and M. H. Clausen, RSC Adv. 2016, 6, 46654-46657; (f) P. Wu, M. Å. Petersen, A. E. Cohrt, R. Petersen, R. Morgentin, H. Lemoine, C. Roche, A. Willaume, M. H. Clausen and T. E. Nielsen, Org. Biomol. Chem. 2016, 14, 69476950

[7] T. Flagstad, C. M. G. Azevedo, G. Min, A. Willaume, R. Morgentin, T. E. Nielsen and M. H. Clausen, Eur. J. Org. Chem. 2018, 5023-5029

[8] (a) A. Cannillo, S. Norsikian,M.-E. Tran Huu Dau, P. Retailleau, B. I. lorga and J.-M. Beau, Chem. Eur. J. 2014, 20, 12133-12143; (b) T. Flagstad, G. Min, K. Bonnet, R. Morgentin, D. Roche, M. H. Clausen and T. E. Nielsen, Org. Biomol. Chem. 2016, 14, 4943-4946; (c) M. Ishoey, R. G. Petersen, M. Å. Petersen, P. Wu, M. H. Clausen and T. E. Nielsen, Chem. Commun. 2017, 53, 9410-9413.

[9] For early accounts, see: N. A. Petasis and I. Akritopoulou, Tetrahedron Lett. 1993, 34, 583-586; G. Dyker, Angew. Chem., Int. Ed. Engl. 1997, 36, 1700. For reviews on the Petasis 3-CR, see: N. A. Petasis in Multicomponent Reactions (Eds.. J. Zhu and H. Bienaymé), Wiley-VCH, Weinheim, 2005, pp. 199-223; R. A. Batey in Boronic Acids (Eds. D. G. Hall), Wiley-VCH, Weinheim, 2005, pp. 279-304; N. R. Candeias, F. Montalbano, P. M. S. D. Cal and P. M. P. Gois, Chem. Rev. 2010, 110, 6169-6193; N. Kumagai, G. Muncipinto and S. L. Schreiber, Angew. Chem., Int. Ed. 2006, 45, 3635-3638; Angew. Chem. 2006, 118, 37173720.

[10] For early accounts, see: O. Diels and K. Alder, Justus Liebigs Ann. Chem. 1928, 460, 98-122. For a review on IMDA, see: K. Takao, R. Munakata and K. Tadano, Chem. Rev. 2005, 105, 4779-4807.

[11] For early accounts, see: O. Mitsunobu and Y. Yamada, Bull. Chem. Soc. Jpn. 1967, 40, 2380-2382; O. Mitsunobu and O. Eguchi, Bull. Chem. Soc. Jpn. 1971, 44, 3427-3430. For reviews on the Mitsunobu reaction see: K. C. K. Swamy, N. N. B. Kumar, E. Balaraman and K. V. P. P. Kumar, Chem. Rev. 2009, 109, 2551-2651; S. Fletcher, Org. Chem. Front. 2015, 2, 739-752.

[12] For early accounts, see: G. C. Fu and R. H. Grubbs, J. Am. Chem. Soc. 1992, 114, 5426-5427; W. J. Zuercher, M. Hashimoto and R. H. Grubbs, J. Am. Chem. Soc. 1996, 118, 6634-6640. For a review on ROM-RCM cascades, see: R. H. Grubbs in Handbook of Metathesis, Vol. 1-3 (Eds. R. H. Grubbs, A. G. Wenzel, D. J. O'Leary, E. Khosravi), Wiley-VCH: Weinheim, 2015. 


\section{Entry for the Table of Contents (Please choose one layout)}

\section{Layout 1:}

\section{FULL PAPER}

An efficient and rapid route to three structurally distinct scaffolds $(\mathrm{A}-\mathrm{C})$ from a single precursor which in turn is available through a one-pot Petasis 3-component-reaction-Diels-Alder cascade reaction. We demonstrate the versatility of the approach through the synthesis of 35 exemplary compounds from the three scaffolds, as well as by the production of 2188 final compounds, which have been included in the Joint European Compound Library of the European Lead Factory.

\section{${ }^{*}$ Petasis/Diels-Alder}

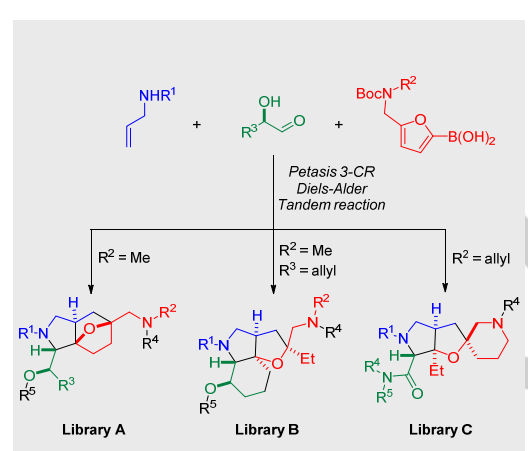

Library synthesis*

Thomas Flagstad, Carlos M. G. Azevedo, Nikolaj S. Troelsen, Geanna

K. Min, Yohan Macé, Anthony

Willaume, Rachel Guilleux, Mélanie

Velay, Karine Bonnet, Remy Morgentin, Thomas E. Nielsen, ${ }^{*}$ Mads H. Clausen*

Page No. - Page No.

Generation of a Heteropolycyclic and $S p^{3}$-Rich Scaffold for Library Synthesis from a Highly

Diastereoselective Petasis/DielsAlder and ROM-RCM Reaction Sequence 